\title{
Important Factors for
}

Accurate Scale-Resolving

Simulations of Automotive

Aerodynamics

\section{Petter Ekman}

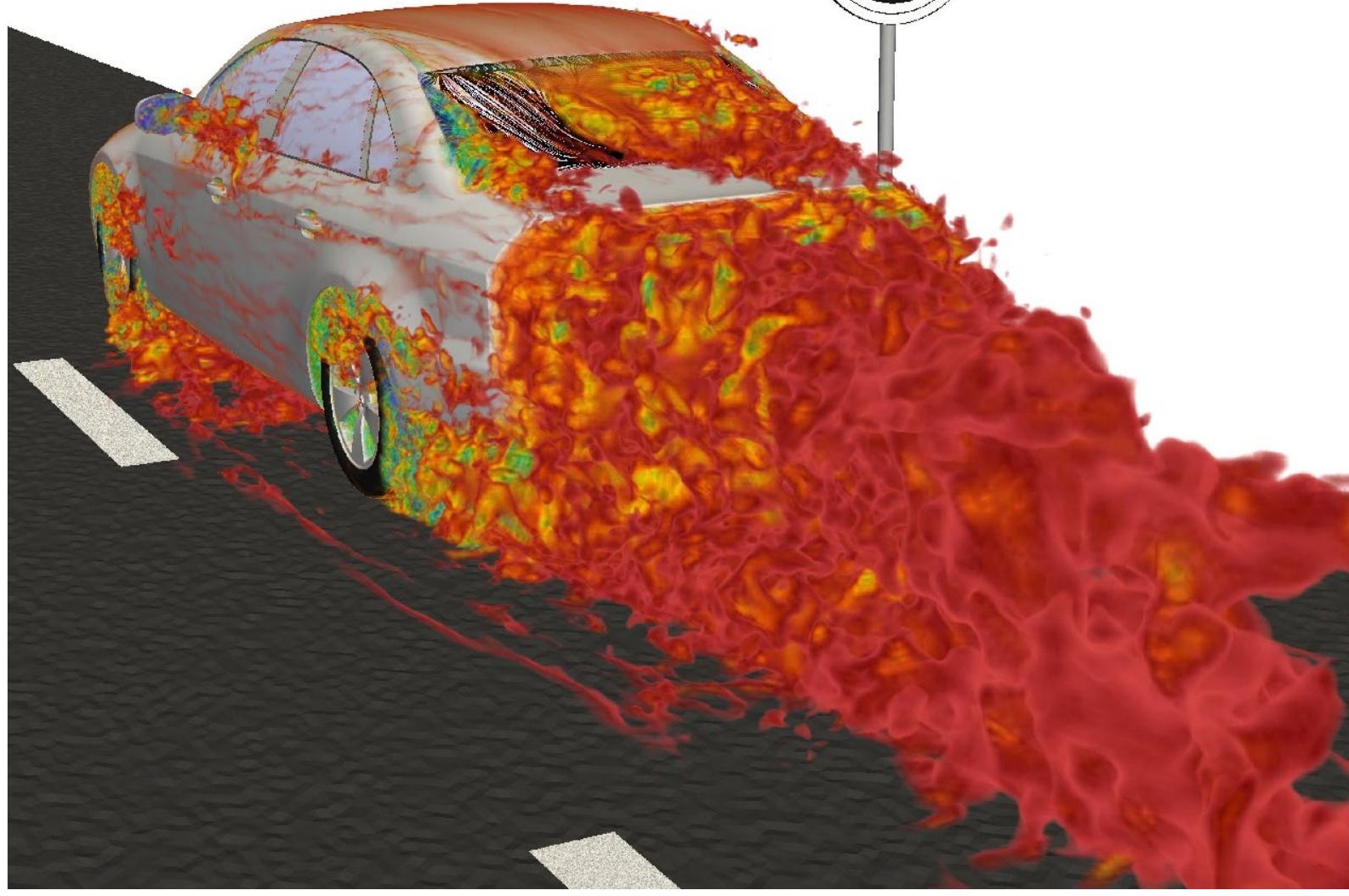


Linköping Studies in Science and Technology

Dissertations, No. 2068

\title{
Important Factors for Accurate Scale-Resolving Simulations of Automotive Aerodynamics
}

\author{
Petter Ekman
}

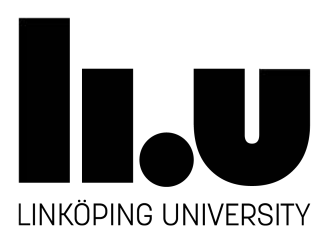

Department of Management and Engineering Division of Applied Thermodynamics and Fluid Mechanics

Linköping University, SE-581 83 Linköping, Sweden

Linköping 2020 
Linköping Studies in Science and Technology. Dissertations, No. 2068

\section{Important Factors for Accurate Scale-Resolving Simulations of Automotive Aerodynamics}

Copyright (C) Petter Ekman, 2020

Typeset by the author in $\mathrm{LAT}_{\mathrm{E}} \mathrm{X} 2 \mathrm{e}$ documentation system.

ISSN 0345-7524

ISBN 978-91-7929-863-0

Printed by LiU-Tryck, Linköping, Sweden 2020

Cover: (Front) Volume rendering of the instantaneous total pressure together with the time-averaged surface streamlines for the rear window of the DrivAer notchback body at $5^{\circ}$ yaw simulated with the SBES $k-\omega$ SST DSM model. (Back) The instantaneous total pressure at the body symmetry line for an aerodynamically improved light truck simulated with the DDES $k-\omega$ SST model. 
I am Batman

Batman 



\section{Abstract}

Road transports are responsible for almost $18 \%$ of the greenhouse gas emission in Europe and are today the leading cause of air pollution in cities. Aerodynamic resistance has a significant effect on fuel consumption and hence the emission of vehicles. For electric vehicles, emissions are not affected by the aerodynamics as such but instead have a significant effect on the effective range of the vehicle.

In 2017, a new measurement procedure was introduced, Worldwide Harmonized Light Vehicles Test Procedure (WLTP), for measuring emissions, fuel consumption, and range. This procedure includes a new test cycle with increased average driving speed compared to the former procedure, which thereby increases the importance of the aerodynamic resistance, as it drastically increases with speed. A second effect is that the exact car configuration sold to the customer needs to be certified in terms of fuel consumption and emissions. The result is that every possible combination of optional extras, which might affect the aerodynamic resistance, needs to be aerodynamically analyzed and possibly improved. From 2021, the European Commission will introduce stricter emission regulations for new passenger cars, with the fleet-wide average lowered to 95 grams $\mathrm{CO}_{2} / \mathrm{km}$, which puts an even higher demand on achieving efficient aerodynamics.

Virtual development of the aerodynamics of road vehicles is today used to a great extent, using Computational Fluid Dynamics, as it enables faster and cheaper development. However, achieving high accuracy for the prediction of the flow field and aerodynamic forces is challenging, especially given the complexity of both the vehicle geometry in itself and the surrounding flow field. Even for a simplified generic bluff body, accurately predicting the flow field and aerodynamic forces is a challenge. The main reason for this challenge of achieving results with high accuracy is the prediction of the complex behavior of turbulence. Scale-resolving simulation (SRS) methods, such as Large Eddy Simulation (LES), where most of the turbulent structures are resolved has in many studies shown high accuracy but unfortunately to a very high computational cost. It is primarily the small turbulent structures within the near-wall region that requires a fine resolution in both space (the mesh) and in time. This fine resolution is the reason for the very high computational cost and makes LES unfeasible for practical use in industrial aerodynamic development at present and in the near future. By modeling the turbulent structures within the near-wall region using a Reynolds-Averaged Navier-Stokes (RANS) model, and resolving the turbulence outside the region with a LES model, a coarser resolution is pos- 
sible to use, resulting in significantly lower computational cost. Which used RANS model is of high importance, and especially how much turbulent viscosity the model generates, as too high values can result in suppression of the resolved turbulence.

The transitioning between the RANS and LES regions have a significant effect on the results. Faster transition enables more resolved turbulence, favorable for higher accuracy, but needs to be balanced with sufficient shielding of the RANS region. If resolving the turbulence occurs within the near-wall region, and the mesh is not sufficiently fine, it can result in poor accuracy.

By increasing the time-step size and disregarding best-practice guides, the computational cost can be significantly reduced. The accuracy is reasonably insensitive to the larger time step sizes until a certain degree, thereby enabling computationally cheaper SRS to achieve high accuracy of aerodynamic predictions needed to meet present and future emission regulations. 


\section{Populärvetenskaplig beskrivning}

Växthusgaser från vägtransporter har ökat stadigt de senaste 30 åren i Europa och står idag för $18 \%$ av de totala utsläppen. Luftmotståndet är en stor del av den totala energiförbrukningen för fordon, framförallt vid högre hastigheter då luftmotståndet ökar med kvadraten av fordonets hastighet.

Allt striktare krav på minskade utsläpp från fordon införs, vilket gör det ännu viktigare att minska luftmotståndet. Detta ställer också högre krav på utvecklingen av fordon, för att kunna nå de lägre utsläppsnivåerna. Mer och mer av utvecklingen för aerodynamik sker idag virtuellt, då det sparar tid och minskar kostnader. För virtuella aerodynamiska analyser används främst CFD (Computational Fluid Dynamics), vilket är numeriska strömningssimuleringar som till exempel kan användas för att beräkna luftflödet runt ett fordon. En stor utmaning med CFD är att nå tillräckligt hög noggrannhet för att alltid kunna lita på resultaten.

Turbulenta flöden är komplexa att prediktera, vilket gör det svårt att få tillräckligt noggranna och tillförlitliga resultat från strömningssimuleringar. Luftflöden runt fordon är nästintill alltid turbulenta och innehåller stora separationer och cirkulerande flöden med kraftig turbulens. För att kunna nå god noggrannhet i estimering av luftmotstånd måste det turbulenta flödet predikteras väl. Det finns flera olika metoder att beräkna turbulensen i strömningsberäkningar, där man kan beräkna eller modellera det turbulenta flödets beteende. Generellt är det svårt att modellera turbulens med hög noggrannhet för alla sorters flöden. Det är framförallt för att de större turbulenta virvlarnas beteende ofta är problemspecifika. Att beräkna turbulensen leder därför oftare till högre noggrannhet men också dessvärre till en högre beräkningskostnad.

För att beräkna turbulensen och nå hög noggrannhet i simuleringen måste en hög upplösning för rum och tid användas, vilket är anledningen till den höga beräkningskostnaden. De minsta turbulenta virvlarna är oftast nära fordonets yta, där den viskösa friktionen mellan ytan och luften saktar in luften. Det är framförallt i detta område som den höga upplösningen i rum och tid behövs i simuleringen för att nå hög noggrannhet. Genom att istället dela upp hanteringen av turbulensen i simuleringen, så att turbulensen modelleras nära och beräknas längre ifrån fordonets yta är det möjligt att sänka beräkningskostnaden men fortfarande bibehålla hög noggrannhet. Hur den här uppdelningen definieras kan dock ha en stor påverkan på resultatens noggrannhet och tillförlitlighet. 


\section{Important Factors for Accurate Scale-Resolving Simulations of Automotive Aerodynamics}

För att systematiskt undersöka hur noggranna nuvarande beräkningsmetoder är för olika turbulenta flöden och prediktering av luftmotstånd har detta undersökts på flera olika fordonstyper, från generiska fordonsmodeller med få särdragna flödesbeteenden till realistiska bilmodeller med hög flödesinteraktion och komplexitet. Detta för att säkerställa att de metoder som används i strömningssimuleringarna klarar av att hantera varierande komplexitet för både förändringar av geometri och flöden och därmed är väl anpassade att nå hög noggrannhet även i industriellt bruk. Flera olika modelleringssätt för turbulens har undersökts på generiska fordonsmodeller. Hög noggrannhet kan för vissa fordonsmodeller uppnås med modellerad turbulens men dessvärre kan aldrig en konsekvent noggrannhet nås. Detta gör det svårt att alltid kunna lita på resultaten från dessa metoder, framförallt för mer realistiska fordonsmodeller. Mycket högre noggrannhet och tillförlitlighet uppnås för alla undersökta fordonstyper när man beräknar turbulensen en bit ifrån fordonets yta. Dessvärre är beräkningskostnaden mycket högre än när man enbart modellerar turbulensen, främst på grund av den nödvändiga upplösningen i tid. Genom att undersöka effekter av att utnyttja en lägre tidsupplösning har beräkningskostnaden kunnat minskas signifikant men ändå med bibehållen hög noggrannhet. Detta skapar en möjlighet att snabbare och mer noggrant kunna förstå den turbulenta flödesfysiken för att fortsätta utveckla bättre och aerodynamiskt effektivare fordon som når utsläppsmålen. 


\section{Acknowledgments}

This thesis was carried out at the Division of Applied Thermodynamics and Fluid Mechanics, Department of Management and Engineering, Linköping University.

I want to thank my main supervisor Matts Karlsson for all your unending encouragement and guidance during this thesis. Part of me wish I had said no to some of your ideas to lower the workload, but the fact is that I would never learned and achieved as much if I had done that, so thank you for all of your exciting ideas! A big thank you to Torbjörn Virdung for all your support, advice, and time you have devoted me. Thank you, Roland Gårdhagen, for all encouragement and feedback during the work.

Special acknowledgment goes to my colleagues at the Division of Applied Thermodynamics and Fluid Mechanics, and especially to Magnus Andersson for continuous discussions and battles over what beer and turbulence modeling approach is best.

Many thanks to Torbjörn Larsson for all your support, continuous encouragements, and interesting motorsport aerodynamics discussions. I truly enjoyed the two years working together with you at Faurecia Creo AB!

This work would not have been possible without the support from the National Supercomputer Center (NSC) at Linköping University. Thank you for all your help, and especially Frank Bramkamp, for your assistance with particularly tricky CFD software issues. I would also like to thank Magnus Ekbladh for continuous professional IT support and fun discussions during coffee breaks.

To all former and present members of the LiU Formula Student team, thanks for all the fun and challenging moments. Unfortunately, from now, I am not allowed by the rules to be involved in the design, but my door will always be open for advice.

Finally, I would like to express my sincere gratitude to my family and friends for always being there when I need them, and for reminding me that there is another reality outside the university. A very special thank you goes to my beloved girlfriend Anna, for all your love and support. Without you, I would never have managed this! 



\section{Funding}

This thesis has been funded partly by the Graduate School at the Department of Management and Engineering at Linköping University and partly from the research grant ETTaero2 - Aerodynamics for greener heavy timber trucks (40281-1) from the Swedish Energy Agency.

The computations were enabled by resources provided by the Swedish National Infrastructure for Computing (SNIC) at National Supercomputer Centre (NSC) partially funded by the Swedish Research Council through grant agreement no. 2016-07213 and by resources provided by LiU at NSC. 



\section{List of Papers}

The following papers are appended and will be referred to by their numerals.

I. Aerodynamic Drag Reduction - from Conceptual Design on a Simplified Generic Model to Full-Scale Road Tests, Petter Ekman, Roland Gårdhagen, Torbjörn Virdung, Matts Karlsson, SAE Technical Paper 2015-01-1543, 2015.

II. Importance of Sub-Grid Scale Modelling for Accurate Aerodynamic Simulations, Petter Ekman, James Venning, Torbjörn Virdung, Matts Karlsson, Accepted for Publication in ASME Journal of Fluids Engineering, March 2020.

III. Aerodynamic Drag Reduction of a Light Truck - from Conceptual Design to Full Scale Road Tests, Petter Ekman, Roland Gårdhagen, Torbjörn Virdung, Matts Karlsson, SAE Technical Paper 2016-01-1594, 2016.

IV. Aerodynamics of an Unloaded Timber Truck - A CFD Investigation Petter Ekman, Roland Gårdhagen, Torbjörn Virdung, Matts Karlsson, SAE International Journal of Commercial Vehicles 9(2):2016, 2016.

V. Accuracy and Speed for Scale-Resolving Simulations of the DrivAer Reference Model, Petter Ekman, Torbjörn Larsson, Torbjörn Virdung, Matts Karlsson, SAE Technical Paper 2019-01-0639, 2019.

VI. Assessment of Hybrid RANS-LES Methods for Accurate Automotive Aerodynamic Simulations, Petter Ekman, Dirk Wieser, Torbjörn Virdung, Matts Karlsson, Submitted for Publication, December 2019. 



\section{Contents}

$\begin{array}{ll}\text { Abstract } & \text { i }\end{array}$

Populärvetenskaplig beskrivning iii

Acknowledgments $\quad$ v

Funding $\quad$ vii

List of Papers $\quad$ ix

1 Introduction $\quad 1$

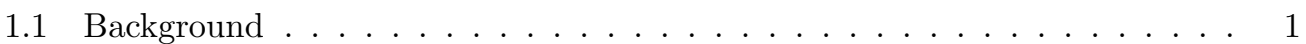

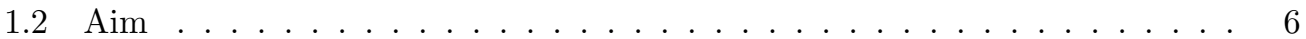

1.3 Limitations . . . . . . . . . . . . . . . . . . . 6

2 Method $\quad 7$

2.1 Automotive Aerodynamics . . . . . . . . . . . . . . . . . 7

2.1 .1 Boundary Layer . . . . . . . . . . . . . . . . . 8

2.1.2 Flow Separation . . . . . . . . . . . . . . 9

2.1.3 Definition of Aerodynamic Forces . . . . . . . . . . . . . . . . . 10

2.2 Investigated Vehicle Geometries and Their Flow Interaction Behavior . . . . 11

2.3 Governing Equations . . . . . . . . . . . . . . . . 12

2.3.1 Computational Fluid Dynamics . . . . . . . . . . . . . 13

2.3.2 Modeling of Turbulence . . . . . . . . . . . . . . . . 13

2.4 Domain and Boundary Conditions . . . . . . . . . . . . . . . . 27 


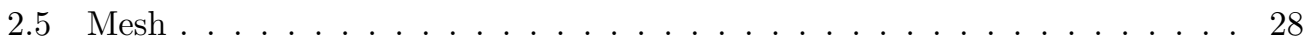

2.6 Solver Settings . . . . . . . . . . . . . . . . . 33

2.7 Experimental Data . . . . . . . . . . . . . . . . 35

2.8 Flow Descriptors . . . . . . . . . . . . . . . . . 37

3 Results and Discussion $\quad 41$

3.1 RANS for Simple Geometries . . . . . . . . . . . . . . . . . 41

3.2 Resolving Turbulence - Less is More . . . . . . . . . . . . . . . . . . 47

3.3 Modeling Turbulence in Specific Regions . . . . . . . . . . . . . . . . . . . 51

3.4 A Reality Check: Drag Reduction . . . . . . . . . . . . . . . . 56

3.5 Reducing Computational Cost . . . . . . . . . . . . . . . 61

3.6 Reliable Accuracy: Hybrid RANS-LES Models . . . . . . . . . . . . . 67

3.7 Calibration of IDDES . . . . . . . . . . . . . . . 73

3.8 Sensitivity of Stress Blending and SGS Modeling . . . . . . . . . . . . . 74

3.9 Accurate Predictions of Small Geometrical Changes . . . . . . . . . . . . 75

3.10 Intuitive Visualization of Turbulence . . . . . . . . . . . . . . . . 77

4 Concluding Remarks $\quad 81$

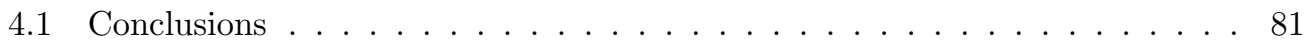

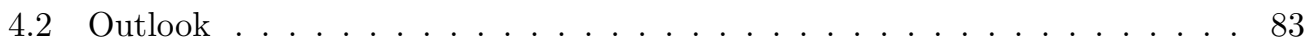

5 Review of Appended Papers $\quad 85$

\section{Appended Papers}

\section{Paper I}




\section{Introduction}

\subsection{Background}

Greenhouse gas emissions from transports have steadily increased during the last 30 years in Europe [1], and in 2017, transports were responsible for $25 \%$ of these greenhouse gas emissions. Of these emissions, almost three quarters are from road transports, Figure 1, and are today the leading cause of air pollution in cities [2]. This has led to several regulations and directives of emissions of new vehicles for automotive manufacturers. Since 2009, the European Commission has introduced legislation for reducing the carbon dioxide $\left(\mathrm{CO}_{2}\right)$ emissions of new passenger cars as a fleet-wide average, and in 2021 the emission target will be lowered to 95 grams $\mathrm{CO}_{2} / \mathrm{km}$. For meeting these emission levels, a corre-

(a)
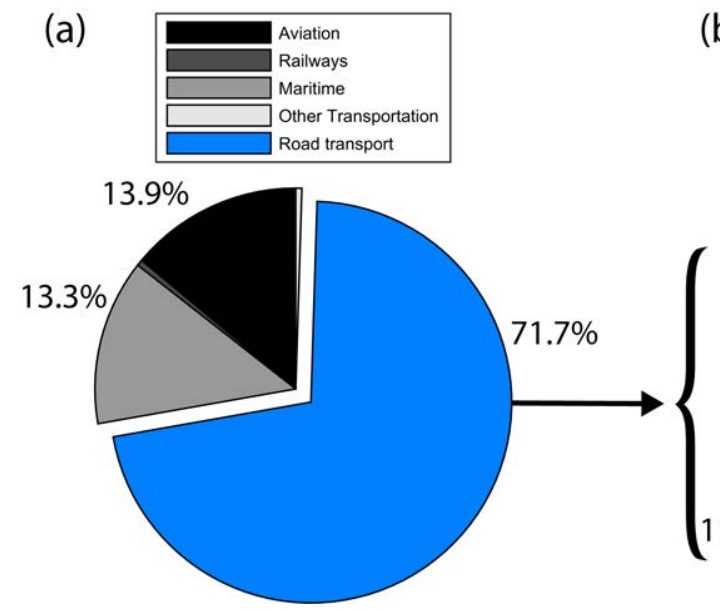

(b)
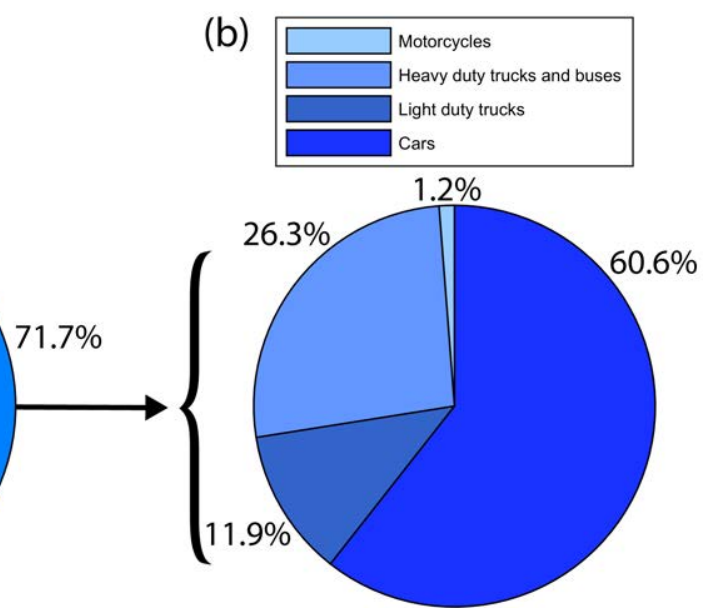

Figure 1: (a) Divisions of greenhouse gas emissions from transports in the European Union 2019. (b) Of these emissions, passenger cars are responsible for most of the emissions, while almost all the rest are from commercial vehicles. 


\section{Important Factors for Accurate Scale-Resolving Simulations of Automotive Aerodynamics}

sponding fuel consumption of around $4.1 \mathrm{~L} / 100 \mathrm{~km}$ and $3.6 \mathrm{~L} / 100 \mathrm{~km}$ are needed for cars with petrol and diesel internal combustion engines, respectively.

Since 2019, $\mathrm{CO}_{2}$ emissions requirements have also been adopted for trucks, with regulations stating that the $\mathrm{CO}_{2}$ emissions should decrease from the 2019 levels with 15 and $30 \%$ to 2025 and 2030, respectively [3]. To meet these regulations, automotive manufacturers are spending significant resources to make vehicles more energy efficient by reducing all forms of losses and resistances. The tractive resistance, $F_{T R}$, is the primary energy loss and can for a driving vehicle be divided into four separate parts and be described as

$$
F_{T R}=F_{\text {Climb }}+F_{\text {Acceleration }}+F_{\text {Rolling }}+F_{\text {Drag }}
$$

Here $F_{C l i m b}$ is the climbing resistance, $F_{\text {Acceleration }}$ the resistance from acceleration, $F_{\text {Rolling }}$ the rolling resistance, and $F_{\text {Drag }}$ is the resistance from aerodynamic drag. Each term in Equation 1 can be explained in more detail as

$$
F_{T R}=m g \sin \alpha+m a+m g \mu_{\text {road }}+0.5 \rho_{\infty} C_{D} A_{\text {ref }} U_{\infty}^{2}
$$

Here $m$ is the mass of the vehicle, $g$ is gravity, $\alpha$ is the inclination of the road, $a$ the acceleration of the vehicle, $\mu_{\text {road }}$ is the rolling resistance coefficient, $\rho_{\infty}$ is the air density, $C_{D}$ is the vehicles aerodynamic drag coefficient, $A_{\text {ref }}$ is the vehicle reference area (typically the projected frontal area of the vehicle) and $U_{\infty}$ is the relative speed between the vehicle and the surrounding air. The first and second terms in Equation 2, are the climbing and acceleration resistances, respectively, and are zero for a vehicle traveling with constant speed on a flat road. This leaves the third and fourth terms, which, respectively, are the resistances from the rolling and aerodynamic drag. Both these resistances are speeddependent, although the rolling resistance stays relatively constant. The aerodynamic drag increase with the square of the speed, making it the dominant resistance at higher speeds. For cars, the aerodynamic drag is the dominating resistance for speeds over $60 \mathrm{~km} / \mathrm{h}$, while for trucks, it is closer to $80 \mathrm{~km} / \mathrm{h}$. Aerodynamic drag, therefore, has a significant effect on the vehicle's fuel consumption and emissions. For electric vehicles, aerodynamic drag does not influence the amount of emissions, but instead the range of the vehicle. In 2019 Audi AG stated that 5 drag counts $\left(\Delta C_{D}=0.005\right)$ correspond to a $2.5 \mathrm{~km}$ in range of their fully electric SUV [4], and in 2016 Kawamata et al. [5] reported that the energy consumption by aerodynamic drag is 4.4 times higher (in respect to the total energy consumption) for electric vehicles compared to Internal Combustion Engine (ICE) vehicles, resulting in even more importance and need for efficient aerodynamics.

In 2017, the Worldwide Harmonized Light Vehicles Test Procedure (WLTP) for measuring the emissions and fuel consumption of cars and light trucks was introduced [6]. WLTP causes two major effects on the importance of aerodynamics on cars. Firstly, the average driving speed in the test cycle is increased to $46 \mathrm{~km} / \mathrm{h}$, which is $12 \mathrm{~km} / \mathrm{h}$ higher compared to the previously used by the New European Driving Cycle (NEDC), thereby increasing the importance of efficient aerodynamics. Secondly, the exact configuration of the car that is sold to a customer needs to be certified in terms of fuel consumption and emissions. This means that any possible variation of optional extras, where some variations might affect the aerodynamic drag, needs to be certified for fuel consumption and emissions. The reason for this is to ensure that the customers should be able to understand better 
what impact specific vehicle configuration will have on fuel consumption and emissions. This results in significantly more aerodynamic analysis and possible optimization for car manufactures, as, e.g., the Volvo XC90 can theoretically be configured in more than 300000 different combinations affecting the aerodynamics of the exterior. Fortunately, significantly reduced, but still, a large number of combinations need to be analyzed to understand the impacts of the changes. Similar measurement procedures and effects on external variations have also been introduced for light and heavy trucks in 2018 and 2019, respectively.

Historically, wind tunnel testing has been the primary tool for aerodynamic analysis and development, Figure 2. During the last 20 years, virtual simulations, and in particularly Computational Fluid Dynamics (CFD), has been increasingly used as an analysis and development tool for aerodynamics. By only requiring a virtual model of the vehicle to perform an aerodynamic evaluation, a much more cost-effective analysis and development process is possible, also making it possible to detect possible design issues earlier in the process. In CFD, not only the aerodynamic forces are available, but also the whole flow field, and in some cases, the sensitivities of the flow field and geometry, resulting in much more data being available than from experimental measurements.

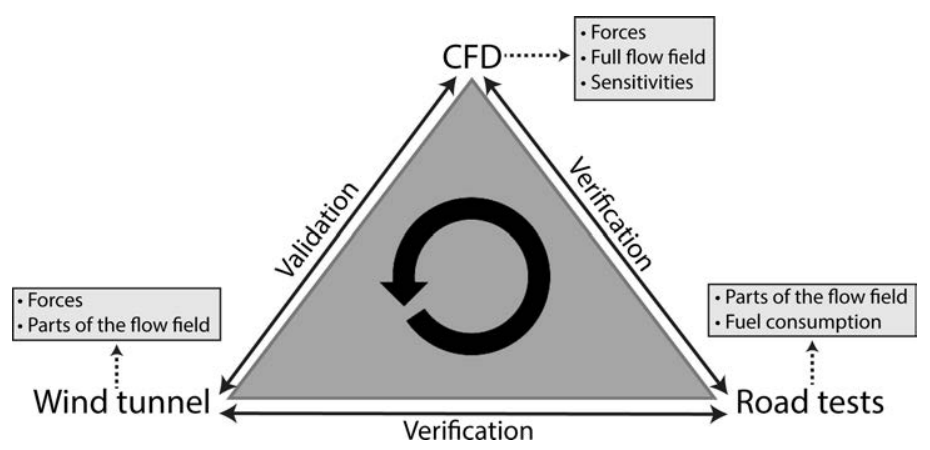

Figure 2: The three mainly used aerodynamic development tools and what kind of data possible to achieve from them. CFD and wind tunnel testing are both simulations of the aerodynamics, but with controlled environments.

Using CFD also makes it possible to simulate conditions that may not be possible to accurately (or at least not easy to) measure in experimental testing, as upstream turbulence, atmospheric boundary layers, cornering, large platoons, etc. Accounting for some of these types of conditions increase possibilities for more accurate predictions of fuel/energy consumption and emissions from vehicles. However, the long-standing problem with CFD simulations is to achieve acceptable accuracy within reasonable computational costs. Wind tunnel testing is also a simulation tool (albeit with correct flow physics) of the aerodynamics, as effects from the wind tunnel setting and measurement equipment may cause uncertainties and errors in the measurements. From wind tunnel measurements, the whole flow field is not possible to acquire, at least within a reasonable time, making it difficult to interpret the results. Roads tests can also be an essential tool in aerodynamic development as the vehicle is exposed to actual real-world conditions. However, the conditions of the real world are uncertain and difficult to control, resulting in significant uncertainties in 


\section{Important Factors for Accurate Scale-Resolving Simulations of Automotive Aerodynamics}

the results, making the results less trustworthy. For reducing the uncertainties, road tests must be performed under a longer time, reducing the practicality of it. However, all these tools are essential in aerodynamic development and important to use together for ensuring that they are accurate and work as supposed to, Figure 2.

For CFD within the automotive sector, mainly steady-state Reynolds-Averaged NavierStokes (RANS) simulations have been used for predicting the aerodynamic performance of vehicles, as it offers reasonable accuracy at a reasonably low computational cost. RANS simulations include several assumptions (some very fundamental, explained in Section 2.3.2) of the turbulent flow behavior, limiting its accuracy. In recent time, many vehicle manufacturers have or are moving to Scale-Resolving Simulation (SRS) methods in order to improve the accuracy deficit. However, the computational cost for SRS is vastly higher than for steady-state RANS simulations but necessary to achieve higher fidelity.

The introduction of WLTP and its effect on geometrical changes, together with the everincreasing amount of vehicle shapes presented by the manufacturers, further increase the number of vehicles and configurations that needs to be aerodynamically evaluated and optimized. The high cost of experimental testing and the need for higher development rates put high demands on accurate CFD simulations, as CFD cannot just be a complement to experimental testing. This has also been acknowledged in the United Nations Economic Commission for Europe (UNECE) global technical regulations for WLTP [7], where it is stated that alternative methods (including CFD) are allowed for determining effects/changes on the drag $\left(\Delta C_{D}\right)$ and thereby effects on fuel/energy consumption and emissions. However, the accuracy of these methods needs to be within $\Delta C_{D} \pm 0.015$, when compared to wind tunnel testing. If CFD is used, the flow field also needs to be validated, so the airflow patterns, including magnitudes of flow velocity and pressures, are shown to match the validation test results [8]. All this puts high demands on accurate but also reasonably fast simulations in order to keep and increase the development rate. CFD is also allowed to be used for determining the drag change $\left(\Delta C_{D}\right)$ on parts and car types where it has shown to be aligned with the accuracy requirements of the WLTP technical regulations [8]. Reliable and consistent accuracy is therefore of great importance, so the same, or at least very similar, methods can be used to determine all types of geometrical changes, thereby lowering the complexity for users.

The design of the vehicles is consistently getting more complex, and the aerodynamics does not only affect the performance of the vehicle but also cooling, aeroacoustic noise, and soiling. This leads to that it is not only enough with single object optimization, as the vehicle designs may need to account for all these effects and not only for efficient aerodynamics. An acceptable correlation of aerodynamic forces is possible to achieve, even though the incorrect flow field is captured. An example of this is seen in Paper VI, where incorrect flow field over the rear window still results in a very accurate prediction of drag when compared to wind tunnel measurements. A good or acceptable correlation would likely not be achieved if other parameters were analyzed with the same methodology, possibly leading to faulty design decisions.

Much research, with a wide variety of approaches, has been done on accuracy on flow simulations of automotive-related bluff bodies. Possibly the most investigated bluff body is the well-known Ahmed body introduced in 1984 [9], featuring a simple shape with a 
variable rear slant angle, Figure 3 panel a. It is known for its complex flow over the rear slant, which is a difficult challenge even for modern and computationally expensive methods. Several other simple vehicle models of different complexity levels exist (well summarized in [10]) and are well suited for investigating the basic flow structures of vehicles, as they only impose some or low interaction of flow features, Figure 3. In 2012, a research group from Technische Universität München released a generic car model for aerodynamic research, called DrivAer [11], Figure 3 panel c. The DrivAer car body is based on two existing mid-size production vehicles from Audi AG and BMW group, to fill the gap between too generic bluff bodies and fully detailed production cars, that are not public. The reason for this was to enable possibilities to investigate more complex and realistic flow phenomena that cannot be well represented by simple generic bodies. Very few publicly available generic bodies (including experimental measurements) exist on the same technical level as the DrivAer car body for the commercial vehicle sector. Here instead, the truck manufacturers are collaborating in extensive research projects for reducing the aerodynamic drag and emissions $[12,13,14]$.

Due to its realistic shape and flow features, the DrivAer car body has, in recent times, become a popular research and validation car body for aerodynamic and CFD investigations, not only for universities and research institutes but also for car manufactures. This has led to more knowledge transfer between academia and the automotive industry, where academic research before often was seen to be performed on too generic bodies for direct relevance to the industry. Several CFD correlation studies have been conducted for the DrivAer car body as well as other simplified bodies, of which many achieve acceptable correlation to measurements. However, no simulation method or approach has yet been established as being the best option for all (or many) types of bodies, car configurations, and types of vehicles, and is the essential aspect behind this thesis.

(a)

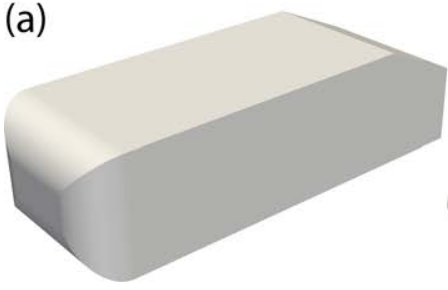

(b)

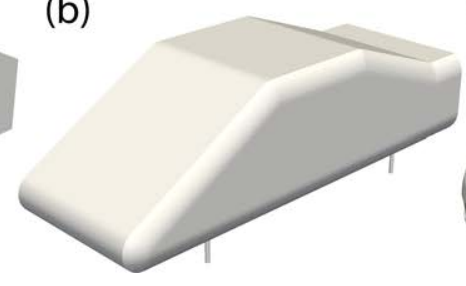

(c)

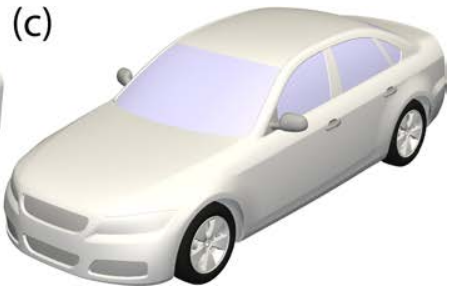

Figure 3: Three generic automotive bluff bodies with different levels of realistic shape and complexity. The Ahmed body (a) is possibly the most studied automotive bluff body despite its simple shape, due to its complex flow field over the rear part of the body. The shape of the SAE reference notchback body (b) provides some similarities to production vehicles but is still a generic bluff body with a relatively generic flow field. The recently introduced DrivAer car body (c) was introduced to bridge the gap between too generic bluff bodies and non-public production vehicles. 


\subsection{Aim}

The focus of this thesis is methods for achieving accurate aerodynamic simulations in automotive engineering, particularly suitable for an industrial process. The aims of this thesis can, therefore, be stated as follows:

- Investigation of how accurate currently available simulation methods are when applied to simple and advanced generic automotive bodies.

- Establish possible simulation approaches to lower the computational cost, and thereby simulation turnaround time, while still achieving a sufficient accuracy.

- Explore reliable accuracy and robustness for each of these methods for a variety of automotive vehicle geometries in order to verify and validate the procedures.

- Apply these procedures to reduce aerodynamic drag for some different types of automotive vehicle geometries.

\subsection{Limitations}

A significant focus of this thesis is on the suitability for industrial application and usability. Only fixed geometries with no internal flow are considered to reduce potential sources of uncertainties and errors. The effect of rotating wheels and internal cooling flow is important for the overall vehicle aerodynamics but requires special modeling techniques, which increases uncertainties. The focus of this thesis is on the ability of currently available simulation methods, and it is therefore chosen to remove these possible uncertainties. In this thesis, a single representative (within the automotive industry) commercially available finite volume solver is used, including several publicly available numerical methods. Other research groups have performed all measurements before the comparison studies in this thesis, and hence no possibilities to affect or suggest measurements have been possible. 


\section{Method}

\subsection{Automotive Aerodynamics}

In terms of fluid dynamics, automotive aerodynamics is bluff bodies in close proximity to the ground. Their shape is complex, which results in complex 3-dimensional flow. Flow separations and reattachments are common on the body, while the base provokes a large turbulent wake.

An important parameter in aerodynamics is the Reynolds number, which is a measure of the ratio of inertial to viscous forces of the flow, Equation 3 [15]. It gives an understanding of the flow physics, especially in the vicinity of the vehicle surface. Automotive vehicles typically operate in moderate to high Reynolds numbers, e.g., a car traveling at 100 $\mathrm{km} / \mathrm{h}$ in normal atmospheric conditions, with a length, $L$, of $5 \mathrm{~m}$ corresponding to a Reynolds number of around $R e_{L}=9.5 \cdot 10^{6}$. These high Reynolds numbers always results in turbulent flow around vehicles.

$$
R e=\frac{U_{\infty} \rho L_{r e f}}{\mu}
$$

Here, $\rho$ is the fluid density, $U_{\infty}$ the free-stream velocity, $L_{r e f}$ the characteristic length and $\mu$ the viscosity of the fluid. The characteristic length is typically the length of the vehicle for cars, and the width or the square root of the frontal area of the vehicle for commercial vehicles.

By using the Reynolds number analogy, the same flow kinematics will be achieved for the same Reynolds number, meaning that same flow field for a full-scale car traveling on the road can be achieved for a scale model in a wind tunnel if the fluid velocity and/or fluid properties are adjusted. For incompressible flow, it, therefore, does not matter which fluid, speed of the fluid or scale of the vehicle is used, as long the same Reynolds number is obtained. Other factors can also affect the flow similarity, as the factors for surface roughness, manufacture tolerances and the scale factors of upstream turbulence must be kept constant. 


\section{Important Factors for Accurate Scale-Resolving Simulations of Automotive Aerodynamics}

\subsubsection{Boundary Layer}

Close to the surface of the vehicle, a thin viscosity dominated region exist, called boundary layer, and has a strong influence on the development of the whole flow field. If no or very low upstream turbulence is present, the beginning of the boundary layer will be laminar but will transition to turbulent downstream when the critical distance is reached, Figure 4. Where the transition occurs mainly depends on the Reynolds number, Equation 3 , but is also affected by the wall surface roughness, free stream turbulence and pressure gradient along the surface $[16,17]$. Transition to turbulent boundary layer typically occurs around $R e_{L}=5 \cdot 10^{5}$ for flow over a flat plate [16, 17], but can be delayed if the flow is accelerated, which stabilizes the flow and suppresses possible instabilities leading to turbulence.
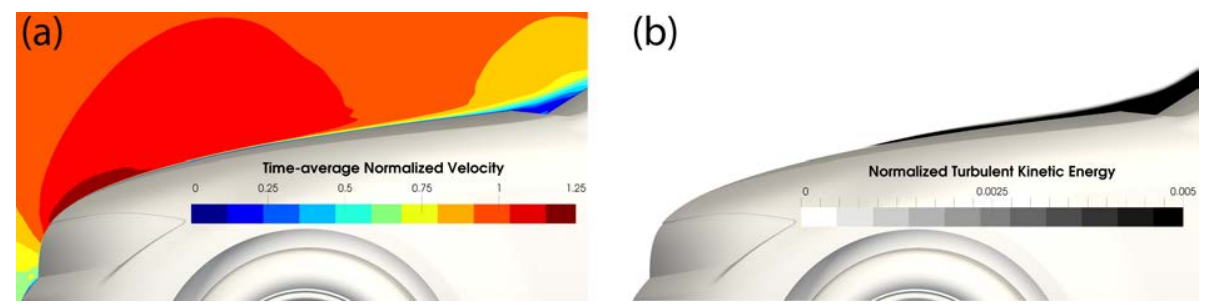

Figure 4: Time-averaged flow velocity distribution (a) and turbulent kinetic energy (b) over the bonnet of a car at $R e_{L}=1.56 \cdot 10^{6}$ simulated with Large Eddy Simulation (LES). Note the build-up of the boundary layer over the bonnet, being laminar in the beginning, and later transition to turbulent as turbulent kinetic energy develops.

A laminar boundary layer behaves steady and results in low skin friction. In contrast, a turbulent boundary layer is unsteady and characterized by rapid fluctuations of velocity and pressure due to motions of near-wall eddies. This results in more mixing of momentum and energy between the layers of the boundary layer, leading to a thicker boundary layer and higher skin friction. The higher momentum in a turbulent boundary layer makes it less prone to separate than a laminar boundary layer, which can be of advantage in vehicle design.

When a laminar boundary layer becomes unsteady, two-dimensional instabilities first occur, called Tollmien-Schlichting waves [18], Figure 5. This can be seen as small instabilities between $x=0.04$ and $0.13 \mathrm{~m}$ in Figure 5 . These instabilities are seen to amplify in the mean flow direction and then evolve into three-dimensional hairpin vortical structures. Above the hairpin vortices, a high shear layer is generated, which intensifies and finally break down into smaller units with a random frequency spectrum [19], resulting in a fully turbulent boundary layer. This effect is seen for the turbulent kinetic energy inside the boundary layer in Figure 4 panel b, where turbulence suddenly occurs.

The location in a fully developed turbulent boundary layers can be described by using the non-dimensional wall unit, $y^{+}$, Equation 4.

$$
y^{+}=\frac{\rho u_{\tau} y}{\mu}
$$


where $u_{\tau}$ is the friction velocity and defined as

$$
u_{\tau}=\sqrt{\frac{\tau_{w}}{\rho}}
$$

Here, $y$ is the wall-normal distance and $\tau_{w}$ the shear force. The boundary layer can then be divided into an inner $\left(y^{+}<50\right)$ and an outer layer. The inner layer can be divided into three regions, the viscous sublayer, buffer layer and the fully turbulent region. The viscous sublayer is located below $y^{+}<5$ and is dominated by viscous stresses. For a car traveling at $100 \mathrm{~km} / \mathrm{h}$, the thickness of the viscous sublayer is in the order of $0.2 \mathrm{~mm}$. The buffer layer is between $5<y^{+}<30$ with the fully turbulent region above.

For a full-scale vehicle traveling at $100 \mathrm{~km} / \mathrm{h}$, the laminar to turbulent transition would occur less than $50 \mathrm{~mm}$ from the leading edge of the vehicle, based on the critical transitional Reynolds number for a flat plate. However, the front part of vehicles typically includes curvatures, which cause flow acceleration and can delay the turbulent transition. Although, still a tiny part of the vehicles is affected by the laminar part for moderate and high Reynolds numbers.

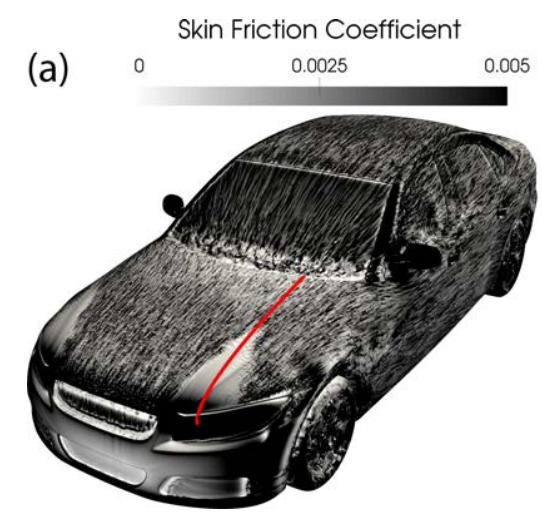

(b)

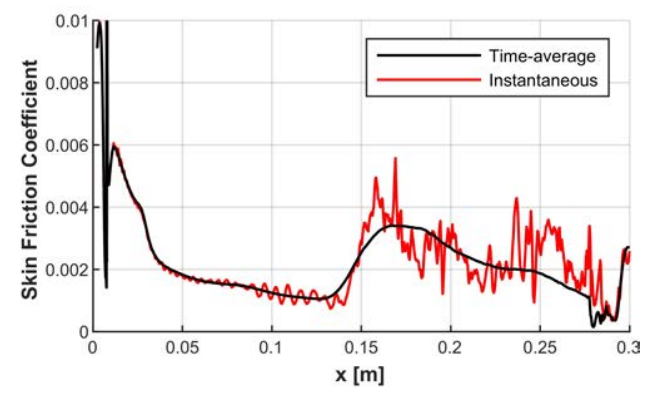

Figure 5: (a) Instantaneous skin friction for the DrivAer car model at $R e_{L}=1.56 \cdot 10^{6}$ simulated with LES. (b) Skin friction along the bonnet (red line in panel a) for both time-averaged and instantaneous skin friction. The high flow velocity causes the high skin friction at the beginning of the bonnet (Figure 4 panel a), while the general decrease of skin friction is caused by the flow velocity reduction along the bonnet. Between $x=0.04$ and 0.13 $\mathrm{m}$ instabilities referred to Tollmien-Schlichting waves are seen, which increase in strength and later $(x \approx 0.14 \mathrm{~m})$ results in turbulent boundary layer and higher skin friction.

\subsubsection{Flow Separation}

The behavior of the boundary layer is strongly dependent on the pressure distribution caused by the flow. If pressure increase in the flow direction occurs, the boundary layer is retarded, especially in the inner layer where the momentum is low. If the pressure increase is too high, this can lead to reversed flow and that the boundary layer separates from the surface. A laminar boundary layer contains less momentum than a turbulent boundary 


\section{Important Factors for Accurate Scale-Resolving Simulations of Automotive Aerodynamics}

layer, and can therefore not withstand as steep pressure gradients as a turbulent boundary layer, and is hence more prone to separate. When dealing with flow with lower Reynolds number, this can have a significant effect on the flow around curvatures, as separation occurs easier, which can significantly affect the flow downstream. Even for moderate and higher Reynolds numbers, this can be important to consider for components downstream of the beginning of the vehicle, as they are directly, or at least partly, subjected to the free stream flow, which is not significantly affected by the upstream flow. The laminar flow behavior can, therefore, be of more importance in those regions. E.g., the mirrors are typically directly subjected to the free stream flow and include small curvatures, making them sensitive to laminar flow behaviors.

Geometries with sharp edges are less affected by the Reynolds number effects, as strong pressure gradients occur at sharp edges, resulting in clearly defined separations. This effect is used as a benefit in Paper II, where a lower Reynolds number is used for investigating flow around the rear of the Ahmed body, which consists of sharp corners.

\subsubsection{Definition of Aerodynamic Forces}

When a vehicle is traveling in a fluid, it is subjected to a force as an effect of the movement of the fluid. This force can be divided into a friction and pressure force component. The friction force acts parallel to the surface and is caused by the viscous effects, while the pressure acts normal to the surface. Pressure drag is the dominating force acting on bluff bodies. The total aerodynamic force can be divided into components based on the vehicle's reference frame. The drag force resists the forward motion of the vehicle, while the lift force acts vertically on the vehicle. The drag force is the main evaluated force in this thesis, as it directly relates to the fuel/energy consumption of the vehicle. Lift can be of importance for aerodynamic stability at higher speeds for passenger cars, while of small, or even no, importance for commercial vehicles. The non-dimensional force coefficients are defined such as

$$
C_{F}=\frac{F}{\left(0.5 \rho_{\infty} U_{\infty}^{2} A\right)}
$$

where $F$ is the force (i.e., the drag, lift and side force), $\rho_{\infty}$ the air density, $U_{\infty}^{2}$ free stream velocity squared and $A$ the projected frontal area of the vehicle.

For cars, around $25 \%, 15 \%$ and $60 \%$ of the drag is generated by the wheels, cooling package and exterior body work, respectively, [16, 20, 21]. For the exterior drag, around $60 \%$ is generated from the front surface and $20 \%$ from the base. For trucks, around $30 \%, 15 \%, 25 \%$ and $30 \%$ is generated from the front surface of the cab, gap between cab and trailer, underbody, and base, respectively [16, 22]. 


\subsection{Investigated Vehicle Geometries and Their Flow Interaction Behavior}

Five different vehicle geometries with different geometrical and flow complexity are investigated in this thesis. Two geometrically simple bodies, the Ahmed and Allan bodies, are generic bodies stipulated during the 1980s for fundamental flow investigations [9, 23]. That said, the flow around these bodies are not necessarily simple. The other three geometries are more geometrically complex (although still less complex than actual production vehicles, as no engine bay nor detailed underbody are included) and include more interaction of flow features. In Figure 6, the vehicle geometries investigated in this thesis are illustrated against their level of flow feature interaction. The two extremes of flow interaction are no/low and high/multi-flow interaction, where the first typically can be the popular test-case backward-facing step or flow around a cylinder, where flow features are relatively isolated. The latter can be the flow around a timber truck, where separating shear layers, vortex shedding, recirculation regions, stagnation zones, etc. are interacting.

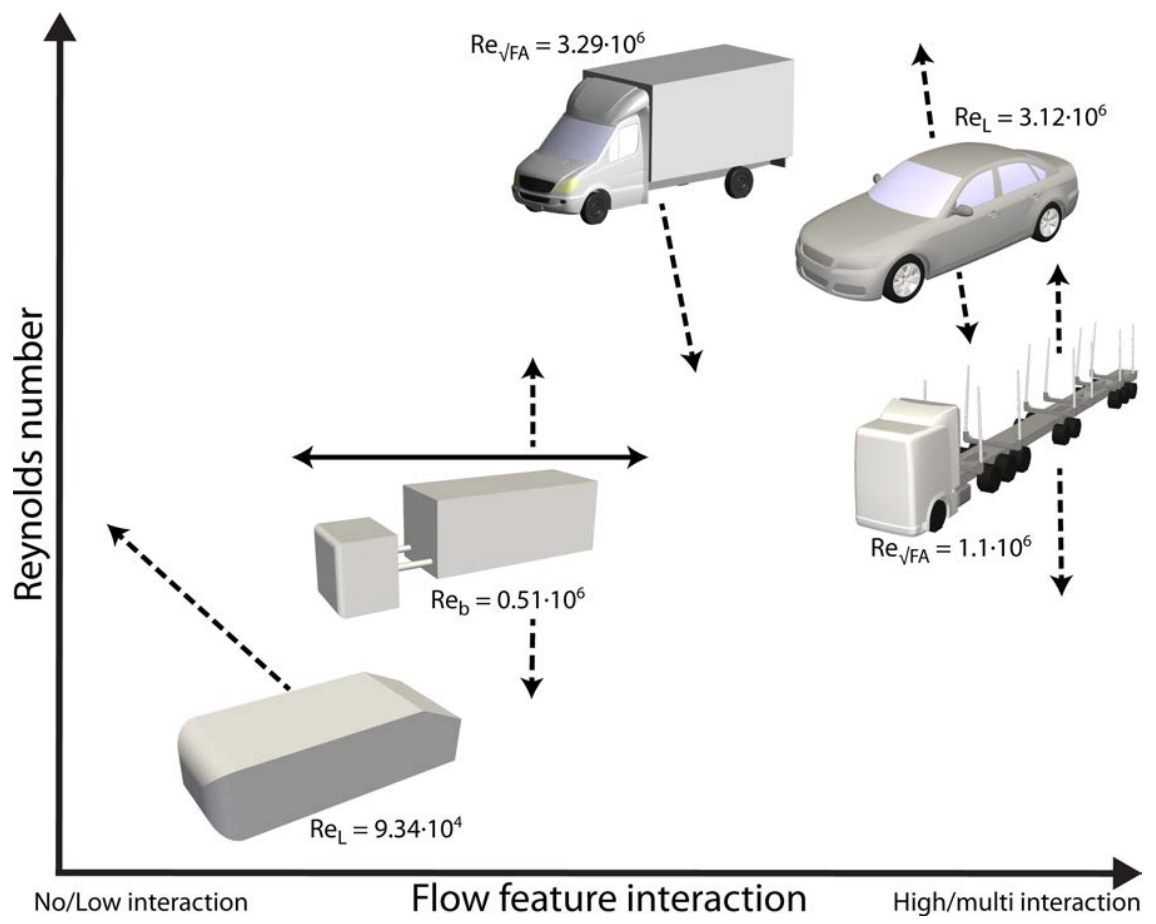

Figure 6: Illustration of the investigated vehicle geometries and their level of flow feature interaction. The Reynolds numbers are presented in the same manner as in the appended papers, as they use different characteristic lengths. The Reynolds number effects on the level of flow interaction are illustrated with dashed arrows. For the Allan body, a solid arrow exists, which indicates the effects of changing gap distance on the flow feature interaction, where a larger gap distance results in less flow interaction. 


\section{Important Factors for Accurate Scale-Resolving Simulations of Automotive Aerodynamics}

The Reynolds number can have a strong effect on the level of flow interaction, and its effect on the vehicle geometries is illustrated with the dashed arrows in Figure 6. The Ahmed body, for example, is designed to have low flow feature interaction between the front and rear part of the body, by having a long straight midsection in between [9]. The rear of the body is less sensitive to Reynolds number effects, as the separation points are clearly defined by the sharp edges. However, a small separation does occur at the front part of the vehicle and increase in size with decreasing Reynolds number, which can interact significantly with the flow over the rear part of the body. Similar behavior also occurs for the stilts supporting the body (Figure 11). Less Reynolds number sensitivity is seen for the other vehicle geometries, as they operate in a Reynolds number range where these effects are limited. For these geometries, high flow feature interaction will always occur, at least within realistic Reynolds numbers, as separating flow always exist. The geometrical set-up of the Allan body makes it slightly different from the other bodies. Here the distance between the front and rear box, representing the cab and swap body, respectively, is designed to be altered in order to investigate different gap distances. This also affects the level of flow interaction (illustrated with the solid arrow in Figure 6), as infinite gap distance results in isolation of the flow around the front and rear box. The level of flow interaction is important to consider, as it has a strong effect on the complexity of achieving accurate simulation results. Hence, for more complex flow, the turbulence modeling needs to be able to account for several types of flow features and how they interact, increasing the complexity of choosing the right model for the problem.

\subsection{Governing Equations}

Three conservation laws govern the motion of a fluid: conservation of mass, conservation of momentum and conservation of energy [19]. The third law, conservation of energy, corresponds to the first law of thermodynamics, which is that the rate of change of energy is equal to the rate of change of heat addition and work done on a fluid particle. In this thesis, incompressible and isothermal fluid properties are assumed, as the investigated flows are for Mach $<0.3$, rendering insignificant compressibility effects, and the energy equation representing the law of energy conservation is neglected [24, 17]. Density and viscosity are, therefore, assumed to be constant. Only the macroscopic behavior of the fluid system is of interest in this thesis, and the fluid can, therefore, be regarded as a continuum [25]. The fluid elements, therefore, represent the average of a large number of fluid molecules in a point in both space and time. The fluid element is the building block in which the conservation of mass, momentum and energy apply to.

The first law, law of conservation of mass, states that mass cannot be created nor destroyed and is for incompressible flow described by the continuity equation, Equation 7.

$$
\frac{\partial u_{i}}{\partial x_{i}}=0
$$

Here, $i=1,2,3$ is each direction in the Cartesian coordinate system, $u_{i}$ is the the velocity component and $x_{i}$ the coordinate directions. 
Conservation of momentum, which describes Newton's second law, is that the rate of change of momentum equals the sum of the forces on a fluid particle, and is for incompressible flow described as

$$
\rho \frac{\partial u_{i}}{\partial t}+\rho u_{j} \frac{\partial u_{i}}{\partial x_{j}}=-\frac{\partial p}{\partial x_{i}}+\frac{\partial \tau_{i j}}{\partial x_{j}}
$$

where

$$
\tau_{i j}=2 \mu S_{i j}
$$

and

$$
S_{i j}=\frac{1}{2}\left(\frac{\partial u_{i}}{\partial x_{j}}+\frac{\partial u_{j}}{\partial x_{i}}\right)
$$

Here, $\rho$ is the fluid density, $p$ pressure, $t$ time and $\mu$ the viscosity of the fluid. For threedimensional flow, which is the only type of flow investigated in this thesis, Equation 8, will be represented with three equations, one for each Cartesian direction, and is together with Equations 9 and 10 known as the Navier-Stokes equation.

\subsubsection{Computational Fluid Dynamics}

Since there exist no general analytic solution to Equations 7 and 8, they need to be solved numerically. To achieve a numerical solution of the equations, a computational domain must be defined. The flow region to be simulated and its interaction with its surroundings are represented by the computational domain. The computational domain is divided into a large set of sub-domains, called cells, which constitute the numerical grid or mesh.

For the finite-volume method, integration of the governing equations (Equation 7 and 8) are then performed over all cells in the domain. Discretization schemes are then used to approximate the resulting integral equations into a system of algebraic equations, which then can be solved in each cell with an iterative method. There exist other methods of doing this, e.g., finite difference, finite element, spectral methods and the lattice Boltzmann method. In this thesis, only the finite-volume method is used.

\subsubsection{Modeling of Turbulence}

Ground vehicles typically operate at a moderate to high Reynolds number, and hence the flow is almost always turbulent. Laminar flow exists, but as described earlier, only a small part of the vehicle is subjected to laminar flow. Turbulent flow has the opposite behavior to laminar flow, as it is irregular, random, chaotic and always three-dimensional [26]. Hence, the turbulence is an important factor (if not the most important) of the flow's behavior around a vehicle.

Turbulent flow around a vehicle consists of eddies with a wide spectrum of spatial and temporal scales. The largest spatial scales typically are in the order of the width or height of the vehicle, while the smallest eddies are very small $\left(<10^{-4} \mathrm{~m}\right)$. For the smallest eddies, called Kolmogorov dissipation scales (based on Kolmogorov's hypothesis [27]), the 


\section{Important Factors for Accurate Scale-Resolving Simulations of Automotive Aerodynamics}

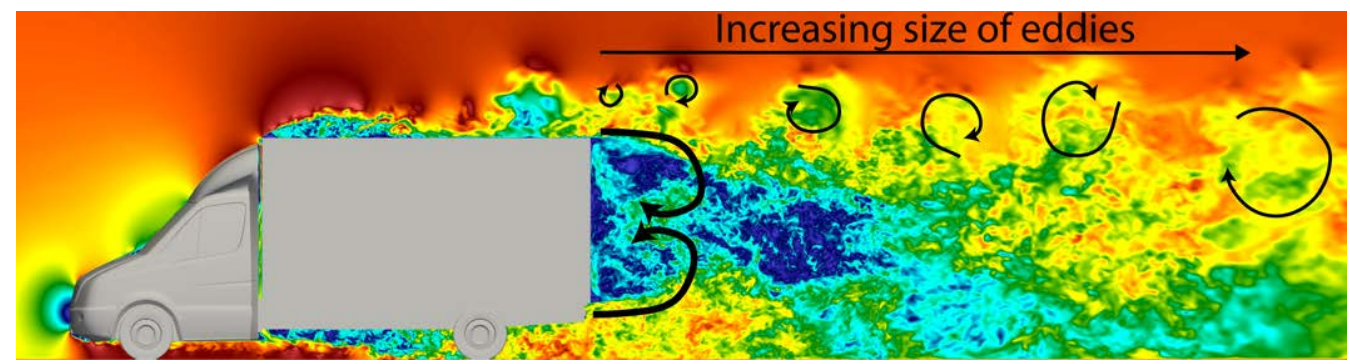

Figure 7: Flow around a light truck where the flow velocity is colored. Red and blue colors indicate high and low velocity, respectively. Severe turbulence with eddies of varying size are seen around the vehicle, especially in the rear wake. An increase of the eddies size occurs downstream of the vehicle, as the smaller eddies dissipate into heat. The thick arrows illustrate the mean recirculating flow in the wake.

viscous effects are becoming dominant, as their kinetic energy dissipates into heat. The small eddies receive their kinetic energy from slightly larger eddies, which in turn receive their energy from even larger eddies and so forth. The largest eddies extract their energy from the mean flow. This transfer of kinetic energy through the different scales of eddies is called the energy cascade, Figure 8.

There exist several methods to simulate turbulent flow numerically, and they can be divided into how much they model and resolve turbulence, where the two most extremes are either to resolve or to model all the turbulent scales, Figure 8. Resolving all turbulent scales can be achieved by directly solving the Navier-Stokes equations, called Direct Numerical Simulation (DNS). The spatial and temporal resolution, therefore, needs to correspond to the Kolmogorov dissipation scales, resulting in extremely high computational costs, even for low Reynolds number flows. In fact, the lowest investigated Reynolds number in this thesis, which is two orders of magnitude lower than a full-scale vehicle traveling at highway speed, is at the time of writing just within grasp the current state of the art DNS simulations. Therefore, turbulence modeling, to at least some part, is necessary in order to reduce the computational cost and be feasible for industrial applications.

In RANS, all the turbulence is modeled, and the time-averaged solution can directly be obtained. This results in that a much coarser spatial resolution can be used for RANS compared to DNS, and results in much lower computational cost. In this thesis, turbulence resolving techniques up to Large Eddy Simulation (LES) have been applied, with the main focus on hybrid RANS-LES methods, which partly model and resolve the turbulence. 


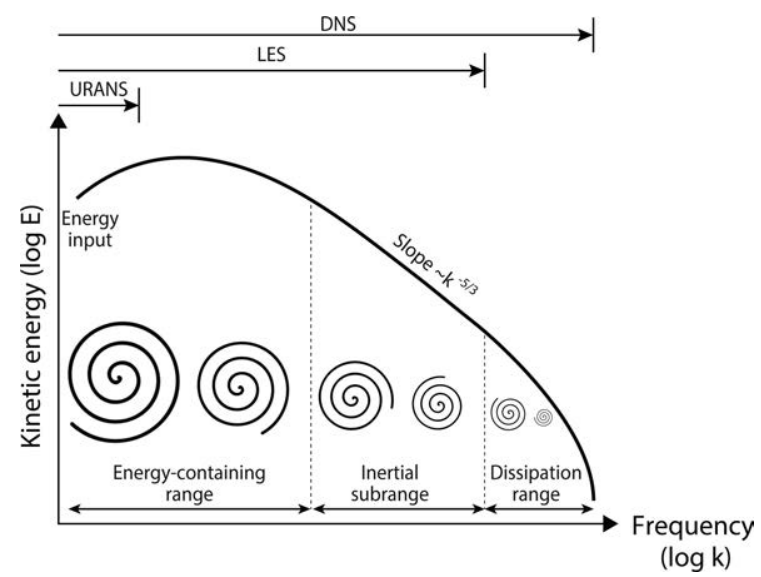

Figure 8: Illustration of a typical energy spectrum of turbulent flow at higher Reynolds numbers. The smaller eddies receive their energy from the larger eddies (which carries most of the energy) until the smallest eddies (within the dissipation range) dissipate into heat. In RANS, all turbulence is modeled, and nothing is resolved and is therefore not included in the figure. DNS, in contrast, resolves all the turbulence, while the idea of LES is to resolve all eddies down to the dissipation range. URANS can only resolve fluctuations of the mean flow, thereby, only a small part of the energy spectrum.

\section{Reynolds-Averaged Navier-Stokes}

By modeling the behavior of all turbulence scales, a much coarser spatial resolution can be used compared to if the turbulence is resolved, as only the gradients of the time-averaged flow field need to be captured. By using the Reynolds decomposition, the instantaneous variables in Equation 7 and 8, can be divided into a time-averaged component, $\bar{\phi}$, and a fluctuating part, $\phi^{\prime}$, as

$$
\phi\left(x_{i}, t\right)=\overline{\phi\left(x_{i}\right)}+\phi^{\prime}\left(x_{i}, t\right)
$$

This can be interpreted as an assumption that the turbulent flow has a statistically steady state. The instantaneous variables in Equation 7 and 8, can then be replaced by this decomposition and subsequently time-averaged (or time-filtered), Equation 12.

$$
\overline{\phi\left(x_{i}\right)}=\frac{1}{\Delta t} \int_{0}^{\Delta t} \phi\left(x_{i}, t\right) d t
$$

This leads to that $\overline{\bar{\phi}}=\bar{\phi}$ and $\overline{\phi^{\prime}}=0$, as time-averaging a time-averaged variable results is the same as the time-averaged variable and the time-averaged of the fluctuating component is zero, respectively, which removes the time derivative. This results in the incompressible Reynolds-averaged Navier-Stokes (RANS) equations, Equation 13 and 14.

$$
\frac{\partial \bar{u}_{i}}{\partial x_{i}}=0
$$




$$
\rho \bar{u}_{j} \frac{\partial \bar{u}_{i}}{\partial x_{j}}=-\frac{\partial \bar{p}}{\partial x_{i}}+\frac{\partial}{\partial x_{j}}\left(2 \mu \bar{S}_{i j}-\rho \overline{u_{j}^{\prime} u_{i}^{\prime}}\right)
$$

where

$$
\bar{S}_{i j}=\frac{1}{2}\left(\frac{\partial \bar{u}_{i}}{\partial x_{j}}+\frac{\partial \bar{u}_{j}}{\partial x_{i}}\right)
$$

Solving these equations results in the time-averaged solution. However, the Reynoldsaveraging introduces the unknown averaged product of the fluctuating component, $\left(-\rho \overline{u_{i}^{\prime} u_{j}^{\prime}}\right)$, called Reynolds stresses, which describes the influence of the turbulent fluctuations on the mean flow. These stresses can have a significant effect on the mean flow and be several orders of magnitude larger than the mean viscous stresses $\left(2 \mu \bar{S}_{i j}\right)$ in fully developed turbulent flow [28]. The introduction of the Reynolds stresses results in more unknowns than equations and leads to a closure problem, which intrinsically requires the introduction of approximations to be solved.

The task of RANS turbulence models is to model the unknown Reynolds stresses, and there exist several proposed methods of doing this. However, no unique solution yet exists, which is suitable for all types of turbulent flow. The most frequently used RANS turbulence models are based on the eddy-viscosity hypothesis Boussinesq proposed in 1877 [29], that the Reynolds stresses are proportional to the mean rates of deformation, Equation 16.

$$
-\left(\overline{u_{i} u_{j}}\right)=2 \nu_{t} \bar{S}_{i j}-\frac{2}{3} k \delta_{i j}
$$

Here, $\nu_{t}$ is the kinematic eddy (or turbulent) viscosity, $\delta_{i j}$ the Kronecker delta and $k$ is the turbulent kinetic energy and is described as

$$
k=\frac{1}{2}\left(\overline{u_{i}^{\prime} u_{i}^{\prime}}\right)
$$

It should be noted that the turbulent kinetic energy has been added to the last term in Equation 16, to avoid the sum of the squared normal Reynolds stresses $\left(\overline{u_{i}^{\prime} u_{i}^{\prime}}\right)$ to be zero for incompressible flow. This implies an isotropic assumption for the Reynolds stresses, which may not always be an accurate assumption, especially for external aerodynamics.

Closure can be obtained by introducing a relationship for the eddy viscosity. There exist several models, with varying complexity, to solve this relationship. In this thesis, four different RANS models have been tested for different geometries and flow conditions. The following section briefly describes the main differences between the models. For a full description of the models, the reader is referred to the original paper for each model, cited in the following sections.

\section{Spalart-Allmaras Model}

The Spalart-Allmaras RANS model is a one-equation model that uses a transport equation to model the behavior of the modified kinematic eddy viscosity, $\tilde{\nu}$, and an algebraic formula for the length scale, $l$. The modified kinematic viscosity equals the eddy viscosity, $\nu_{t}$, 
except in the near-wall region where the modified eddy viscosity is related to a walldamping function, Equation 18 [30].

$$
\nu_{t_{S A}}=\tilde{\nu} f_{v 1}
$$

Here, $f_{v 1}$ is the wall-damping function and equals

$$
f_{v 1}=\frac{\chi^{3}}{\chi^{3}+C_{\nu 1}^{3}}
$$

Here $\chi=\tilde{\nu} / \nu$, where $\nu$ is the kinematic viscosity of the fluid, and $C_{\nu 1}$ is a model constant. The length scale, $l$, is determined by $l=\kappa y$, where $\kappa$ is the von Kármán constant and $y$ the wall distance and affects the rate of dissipation of $\tilde{\nu}$. For complex geometries, this can lead to difficulties in defining the length scale, making it unsuitable for general internal flows [19]. However, the model is accurate for flows with boundary layers subjected to adverse pressure gradient, typically existing in external aerodynamic applications.

\section{Realizable $k-\varepsilon$ Model}

The standard $k-\varepsilon$ model (first introduced in 1974 by Launder and Spalding [31, 32, 33]) is a two-equation model, with a transport equation for the turbulent kinetic energy, $k$, and another for the dissipation rate, $\varepsilon$. The focus of the $k-\varepsilon$ model is on the mechanism affecting the turbulent kinetic energy [19, 34], as the $k$-equation is derived from the exact equation. The $\varepsilon$-equation is obtained from measurements and physical reasoning. The turbulent viscosity is computed from a combination of the two equations for $k$ and $\varepsilon$ and is defined as

$$
\nu_{t_{k-\varepsilon}}=C_{\mu} \frac{k^{2}}{\varepsilon}
$$

where, $C_{\mu}$ is a model constant. The model assumes fully developed turbulent flow, where the effects of the molecular viscosity are negligible. The standard $k-\varepsilon$ model is, therefore, only valid for fully turbulent flow, and the model is known to struggle for circulating flow, weak shear layers and flows containing strong adverse pressure gradients.

Several variations of the $k-\varepsilon$ model exist, and possibly the most popular is the realizable version introduced by Shih [35]. It differs from the standard $k-\varepsilon$ model in two significant ways. The normal stress $\overline{u_{i}^{\prime 2}}$ in the standard $k-\varepsilon$ model can become negative, and nonrealizable, when the strain is large, which not is a physical turbulent behavior. This is solved in the realizable $k-\varepsilon$ model by making the $C_{\mu}$ a variable, Equation 21 .

$$
C_{\mu}=\frac{1}{A_{0}+A_{S} \frac{k U^{*}}{\varepsilon}}
$$

Here, $A_{0}$ and $A_{S}$ are model constants, while $U^{*}$ depends on the mean rate-of-rotating tensor and strain rate tensor. The $\varepsilon$-equation is also modified, so it is based on the dynamic equation of the mean-square vorticity fluctuation. These modifications result in a $k-\varepsilon$ model that is suitable for a more variety of flows than the standard version, as improvements are seen for homogeneous shear flows, rotating flows and separated flows. 


\section{Important Factors for Accurate Scale-Resolving Simulations of Automotive Aerodynamics}

\section{$k-\omega$ SST Model}

In 1988, Wilcox introduced the two-equation empirical model $k-\omega$, which model the transport of the $k$ and the specific dissipation rate $\omega$ (where $\omega=\varepsilon / k$ ) [36]. The $k-\omega$ model has shown good accuracy for the near-wall region but is very sensitive for the solution of $k$ and $\omega$ in the free-stream. In 1992 Menter extended the model into the $k-\omega$ Shear Stress Transport (SST) RANS model, which utilizes the two two-equation RANS models, $k-\omega$ and $k-\varepsilon$, for the near-wall and fully turbulent region, respectively [37]. By utilizing the $k-\varepsilon$ model in the turbulent region further away from the wall, the free-stream sensitivity is removed for the $k-\omega$ model together with the near-wall accuracy problems of the $k-\varepsilon$ model. For the standard $k-\omega$ model the eddy viscosity is defined as

$$
\nu_{t_{k-\omega}}=\frac{\omega}{k}
$$

In the $k-\omega$ SST model the standard $k-\omega$ and the $k-\varepsilon$ (which is transformed into a $k-\omega$ formulation) are multiplied by a blending function. The blending function is one for the near-wall region, which activates the standard $k-\omega$ model, and zero further away from the wall, which activates the transformed $k-\varepsilon$ model. This together with accounting for the transport of turbulent shear stress leads to the eddy viscosity formulation which is

$$
\nu_{t_{S S T}}=\frac{k}{\omega} \frac{1}{\max \left(\frac{1}{a^{*}}, \frac{S F_{2}}{a_{1} \omega}\right)}
$$

where $a^{*}$ is a low-Reynolds number correction which suppress the eddy viscosity [38], $\mathrm{S}$ the strain rate magnitude $\left(\sqrt{2 S_{i j} S_{i j}}\right), a_{1}$ a model constant and $F_{2}$ the blending function $[37]$.

\section{Reynolds Stress Model}

An alternative approach to the Boussinesq hypothesis (Equation 16) is to model each of the Reynolds stresses, called Reynolds stress modeling (RSM). The Reynolds stresses are then modeled with transport equations together with an equation for the dissipation rate, leading to seven more equations to solve in order to close the problem. The model can, therefore, account for anisotropic behavior of turbulence and more complex flows than the models based on the isotropic eddy-viscosity hypothesis. However, the RSM models are still limited by the closure assumptions needed for specific terms in the transport equation of the Reynolds stresses. In particular, the modeling of the pressure-strain and dissipation-rate is challenging and is the main reason for the limited fidelity of the models. In this thesis, the Linear Pressure-Strain Model by Gibson and Launder [39] is used.

\section{RANS Near-wall Treatments}

For the realizable $k-\varepsilon$ model simulations, two types of wall modeling have been used, the Standard Wall Function (Std WF) and Enhanced Wall Treatment (EWT). The nearwall modeling can significantly impact the solution, as the walls are the primary source of 
turbulence and vorticity. As described in the Boundary layer section (2.1.1), the near-wall region has strong gradients and turbulent mixing, which are important to capture either by resolving or modeling them. The latter is done with wall functions, where the law-ofwall behavior of the mean velocity and the near-wall turbulent behavior is modeled.The Std WF is based on the work of Launder and Spalding [33], where the near-wall mean velocity is described as

$$
U^{*}=\frac{1}{\kappa} \ln \left(E y^{*}\right)
$$

where $\kappa$ is the von Kármán constant, $E$ an empirical constant, and $y^{*}$ the dimensionless distance from the wall, which is similar to the dimensionless wall distance $y^{+}$in equilibrium turbulent boundary layers [40]. If the first wall adjacent grid point (seen in the wall-normal direction) is positioned for a $y^{*}$ below 11.225 , the near-wall mean velocity is instead linearly described as

$$
U^{*}=y^{*}
$$

The $k$-equation is solved near the wall, while the turbulent kinetic production is based on the logarithmic law. The $\varepsilon$-equation is not solved in the wall-adjacent cells as instead is modeled with

$$
\varepsilon=\frac{C_{\mu}^{\frac{3}{4}} k^{\frac{3}{2}}}{\kappa y}
$$

where $y$ is the wall distance.

The EWT is a $y^{+}$insensitive near-wall model for the $\varepsilon$-equation that combines a two-layer model with enhanced wall functions [40]. If the first wall adjacent grid point is positioned within the viscous sublayer $\left(y^{+}<5\right)$, the EWT uses a two-layer model that specifies both $\varepsilon$ and the $\mu_{t}$. $\varepsilon$ is then modeled with the one-equation model proposed by Wolfshtein [41]. The behavior of $\mu_{t}$ is described in two layers with a smooth transition in between by using a blending function [40, 42, 43]. From the fully turbulent region, both $\varepsilon$ and $\mu_{t}$ are modeled with the RANS model equations. This results in that the EWT is less sensitive to $y^{+}$and better predicts strong pressure gradients where the Std WF performance can be poor. A similar method is also used for ensuring a $y^{+}$insensitive behavior for the Spalart-Allmaras and $k-\omega$ SST models [40].

\section{Unsteady RANS}

RANS turbulence models simulations can also run in transient mode, by retaining the transient term of the Navier-Stokes equations, resulting in Unsteady RANS (URANS).

In URANS, the $\Delta t$ in the Equation 12 should be much smaller than the resolved time scales, meaning that motions larger than the simulation time-step is resolved, which is the unsteadiness of the mean flow, while the turbulent fluctuations are time-filtered out and modeled [44]. However, this requires significant scale separation, where resolved timevariations are of much lower frequency than the turbulence, which rarely is present and causes the URANS concept to be unclear [34, 45]. Large time-steps, which only can resolve the fluctuations of the mean flow, are therefore used in URANS simulations. For 


\section{Important Factors for Accurate Scale-Resolving Simulations of Automotive Aerodynamics}

bluff bodies, this can sometimes be beneficial in order to capture the unsteadiness of nonturbulent origin. This can typically be from moving geometry, time-varying boundary conditions, and unsteady flow behavior e.g., vortex shedding behind a bluff body [34], seen in the top part of the wake in Figure 7.

\section{Scale-Adaptive Simulation}

The Scale-Adaptive Simulation (SAS) turbulence model is developed by Menter et al. [46] and is an improved URANS formulation, with possibilities to switch to an LES-like behavior in unsteady regions. The SAS model is considered as a URANS model due to that no explicit filter or grid dependency exists in its formulation [47].

In the $k-\omega$ SST-SAS model [48], used in this thesis, the model dynamically adjusts to resolve turbulent structures by use of the von Kármán length scale, which decreases when local unsteadiness occurs. When this occurs, an additional source term in the $\omega$ equation ensures that $\omega$ increases, which results in decreased eddy viscosity, and that more unsteadiness can be resolved.

\section{Large Eddy Simulation}

The concept of Large Eddy Simulation (LES) is built upon the idea that the larger eddies transport most of the momentum, mass and energy, and are more problem-dependent than the smaller eddies. The larger eddies can also be very anisotropic and are, as seen for RANS turbulence models, difficult to model accurately. However, the behavior of the smaller eddies is more universal and can, according to the Kolmogorov hypothesis, be considered statistically isotropic, and therefore easier to model accurately. This makes it possible to use simpler models (than RANS turbulence models) to model the effect of the smaller eddies and still achieve high accuracy [45]. In comparison to URANS, LES is meant to resolve the majority of the turbulent motions, Figure 8, and the border between resolving and modeling is decided by the local grid spacing. The turbulent scales that are smaller than the grid spacing, $\Delta$, are modeled and referred to as Sub-Grid Scales (SGS). In LES the Navier-Stokes equations are spatially filtered (space-filtered), Equation 27 and 28.

$$
\begin{gathered}
\frac{\partial \widetilde{u}_{i}}{\partial x_{i}}=0 \\
\frac{\partial \tilde{u}_{i}}{\partial t}+\frac{\partial \widetilde{u}_{i} \widetilde{u}_{j}}{\partial x_{j}}=-\frac{1}{\rho} \frac{\partial \widetilde{p}}{\partial x_{i}}+\frac{\partial}{\partial x_{j}}\left(\nu \frac{\partial \widetilde{u}_{i}}{\partial x_{j}}\right)+\frac{1}{\rho} \frac{\partial \tau_{i j}}{\partial x_{j}}
\end{gathered}
$$

Here $\widetilde{u}_{i}$ and $\widetilde{p}$ are the filtered variables for the resolved velocity and pressure, respectively. $\tau_{i j}$ is the SGS turbulent stress and is defined as

$$
\tau_{i j}=\widetilde{u_{i} u_{j}}-\widetilde{u}_{i} \widetilde{u}_{j}
$$

Like RANS, the SGS turbulent stresses are unknown, and closure of the equations is needed. In this thesis, this is done by use of SGS models, which like in many RANS 
models formulations are based on the Boussinesq hypothesis of isotropic turbulence

$$
\tau_{i j}=\frac{1}{3} \tau_{k k} \delta_{i j}-2 \mu_{S G S} \widetilde{S}_{i j}
$$

where $\mu_{S G S}$ is the SGS eddy viscosity and $\widetilde{S}_{i j}$ is the resolved rate-of-strain tensor, and is defined as

$$
\widetilde{S}_{i j}=\frac{1}{2}\left(\frac{\partial \widetilde{u}_{i}}{\partial x_{j}}+\frac{\partial \widetilde{u}_{j}}{\partial x_{i}}\right)
$$

The isotropic part of the SGS stresses $\tau_{k k}$ is not modeled, but instead added to the filtered static pressure term, resulting in $\widetilde{P}=\widetilde{p}+\tau_{k k} / 3$. Combining Equation 28 and Equation 30 yields the LES equations with the SGS kinematic eddy viscosity $\left(\nu_{S G S}=\mu_{S G S} / \rho\right)$

$$
\frac{\partial \widetilde{u}_{i}}{\partial t}+\frac{\partial \widetilde{u}_{i} \widetilde{u}_{j}}{\partial x_{j}}=-\frac{1}{\rho} \frac{\partial \widetilde{P}}{\partial x_{i}}+\frac{\partial}{\partial x_{j}}\left(\left(\nu+\nu_{S G S}\right) \frac{\partial \widetilde{u}_{i}}{\partial x_{j}}\right)
$$

As the smallest eddies in LES are not resolved, the correct dissipation rate is obtained with SGS models, that introduce the SGS eddy viscosity. In this thesis, three SGS models have been used: the Smagorinsky-Lilly (SM) model, the Dynamic Smagorinsky-Lilly (DSM) model and the Wall-Adapting Local Eddy-Viscosity (WALE) model. Following is a short description of each SGS model.

\section{Smagorinsky-Lilly SGS Model}

In the SM model, first proposed by [49], the kinematic SGS eddy viscosity is modeled as

$$
\nu_{S G S_{S M}}=L_{s}^{2}|\widetilde{S}|
$$

where $|\bar{S}|=\sqrt{2 \widetilde{S}_{i j} \widetilde{S}_{i j}}$ and $L_{s}$ is the mixing length for the SGS, and is defined as

$$
L_{s}=\min \left(\kappa d, C_{s} \Delta\right)
$$

Here, $\kappa$ is the von Kármán constant, $d$ the distance to the closest wall, $C_{s}$ the SM model constant and $\Delta$ is the grid-filter length. The SM SGS model constant is in this thesis equal to 0.1 , which has been used for several studies involving flow around bluff bodies $[50,51,52,53]$. The grid-filter length is calculated from the cube root of each cell volume in the mesh, $\Delta=V^{1 / 3}$. Since the model is not able to provide zero eddy viscosity in laminar flow, it needs a wall-damping function. This is achieved by $\kappa d$ in Equation 34, which ensures that the mixing length goes toward zero at the wall and resulting in the same behavior for the SGS eddy viscosity (Equation 33). 


\section{Important Factors for Accurate Scale-Resolving Simulations of Automotive Aerodynamics}

\section{Dynamic Smagorinsky-Lilly SGS Model}

The DSM SGS model is based upon the SM SGS model but with a modification of the SM SGS model constant, $C_{s}$ [54]. Instead of being a constant value, it is dynamically calculated as a function of space and time. It uses information from the smaller scales of the resolved field, by using a test-filter to separate the smaller resolved scales. The test-filter size, $\widehat{\Delta}$, used in this thesis, is twice the size of the grid-filter length [54]. This test-filter is used on the Navier-Stokes equations and results in a subtest-scale stress, Equation 35.

$$
T_{i j}=\widehat{{\widehat{u_{i} u_{j}}}_{j}}-\widehat{\widetilde{u}}_{i} \widehat{\widetilde{u}}_{j}
$$

Here " $\leadsto "$ denotes the test-filter variables. In [54] it was seen that $\tau_{i j}$ and $T_{i j}$ are related to each other and can be used to determine the Leonard stresses, Equation 36, which can be interpreted as the resolved stresses of the smaller scales between the grid and test-filter.

$$
L_{i j}=T_{i j}-\tau_{i j}
$$

From this an ad-hoc solution of the DSM models variable, $C_{d s}$, can be defined [54]

$$
C_{d s}=\left(\frac{L_{i j} M_{i j}}{M_{i j} M_{i j}}\right)^{1 / 2}
$$

where $M_{i j}=-2\left(\widehat{\Delta}^{2}|\widehat{\widetilde{S}}| \widehat{\widetilde{S}}_{i j}-\widetilde{\Delta^{2}} \widehat{|\widetilde{S}| \widetilde{S}_{i j}}\right) \cdot C_{d s}$ varies both in space and time and is in this thesis limited between 0 and 0.23 to avoid numerical instabilities [49, 55]. The SGS eddy viscosity and mixing length is calculated in the same manner as for the SM SGS model, except the dynamic model constant, Equation 38.

$$
L_{s}=\min \left(\kappa d, C_{d s} \Delta\right)
$$

This makes the DSM SGS model to have correct near-wall behavior, as the eddy-viscosity automatically goes to zero in laminar flows $[54,55]$.

\section{Wall-Adapting Local Eddy-Viscosity SGS Model}

Another approach of achieving correct near-wall behavior and zero eddy viscosity in laminar flow is the WALE SGS model proposed by Nicoud e.t al. [56]. The SGS kinematic eddy viscosity is defined as

$$
\nu_{S G S_{W A L E}}=L_{s}^{2} \frac{\left(S_{i j}^{d} S_{i j}^{d}\right)^{3 / 2}}{\left(\widetilde{S}_{i j} \widetilde{S}_{i j}\right)^{5 / 2}+\left(S_{i j}^{d} S_{i j}^{d}\right)^{5 / 4}}
$$

where $S_{i j}^{d}$ is the traceless symmetric part of the square of the velocity gradient tensor and is defined as

$$
S_{i j}^{d}=\widetilde{S}_{i k} \widetilde{S}_{k j}+\widetilde{\Omega}_{i k} \widetilde{\Omega}_{k j}-\frac{1}{3} \delta_{i j}\left(\widetilde{S}_{m n} \widetilde{S}_{m n}-\widetilde{\Omega}_{m n} \widetilde{\Omega}_{m n}\right)
$$


where $\widetilde{\Omega}$ is the filtered vorticity and $\widetilde{S}$ the filtered strain-rate. The mixing length is defined similar to SM and DSM SGS models, but with the WALE SGS model constant, Equation 41.

$$
L_{s}=\min \left(\kappa d, C_{w} \Delta\right)
$$

In this thesis, the $C_{w}$ equals 0.325, after showing superior accuracy in the used solver [57]. As for the DSM SGS model, the WALE SGS model also results in zero SGS eddy viscosity in laminar flow and achieves correct near-wall behavior, as the formulation of the SGS eddy viscosity (Equation 39) acts as an automatic damping function.

\section{LES Near-Wall Treatment}

All the meshes used for LES in this thesis are fine enough to resolve the laminar sublayer, and hence the wall shear stress is obtained from the laminar stress-strain relationship, Equation 42.

$$
\bar{u}^{+}=y^{+}
$$

Here $\bar{u}^{+}$is the non-dimensional velocity and $y^{+}$the non-dimensional wall distance, as earlier defined in Equation 4.

\section{Hybrid RANS-LES models}

The cost of LES simulations is high, especially for high Reynolds numbers, as both high spatial and temporal resolution are needed to resolve the small turbulent structures, which decrease in size with increasing Reynolds number. These small turbulent structures, are mainly located in the near-wall region, and hence a fine mesh is needed in the near-wall region to resolve them accurately. However, further away from the wall, the turbulent structures are larger and do not require as fine mesh in the near-wall region to be adequately resolved. Hybrid RANS-LES methods are built upon the idea of using RANS for modeling the behavior of the small turbulent structures in the near-wall region, and LES for resolving the larger turbulent structures further away from the wall. Thereby, significantly reducing the computational cost compared to LES and as well as increasing the accuracy compared to RANS, Figure 9.

In RANS, URANS and LES the Reynolds stresses or SGS stresses are substituted with eddy viscosity models, which results in that the URANS and LES momentum equations are identical, even though the URANS and LES are space and time-filtered, respectively. The difference, therefore, only lies in the amount of eddy-viscosity the underlying turbulence model provides, making it possible to formulate turbulence models that can switch between RANS and LES, without any formal change of the momentum equations. 


\section{Important Factors for Accurate Scale-Resolving Simulations of Automotive Aerodynamics}

In this thesis, five hybrid RANS-LES models are investigated: Detached Eddy Simulations (DES), Delayed Detached Eddy Simulation (DDES), Improved Delayed Detached Eddy Simulation (IDDES), Shielded Detached Eddy Simulation (SDES) and Stress Blended Eddy Simulation (SBES). There exist several other hybrid RANS-LES methods, which are outside of the scope of this thesis. Following is a brief description of the main differences between the investigated hybrid RANS-LES models. A detailed review of hybrid RANS-LES methods can be found in [58, 47].

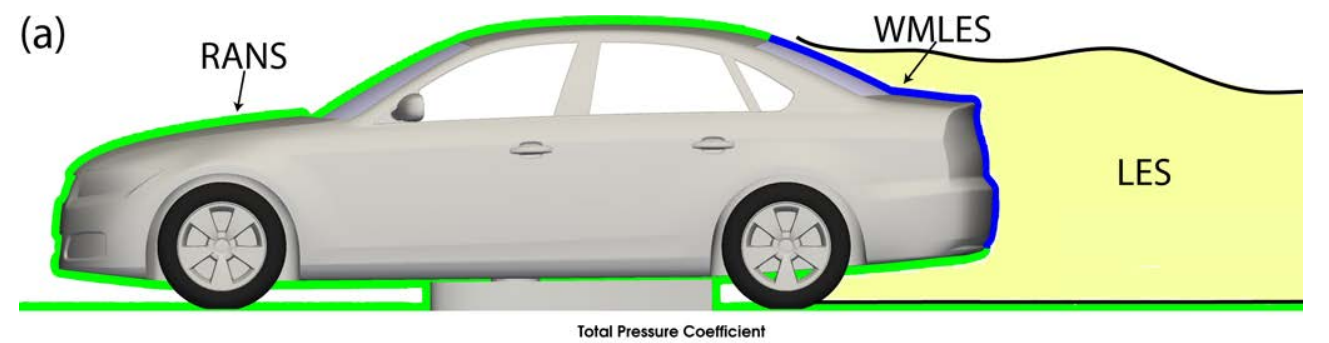

(b)

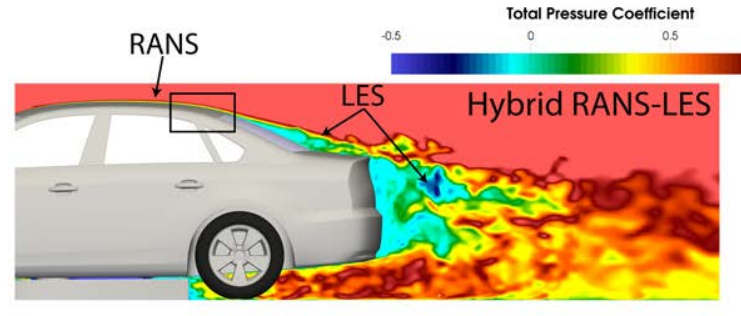

(c)
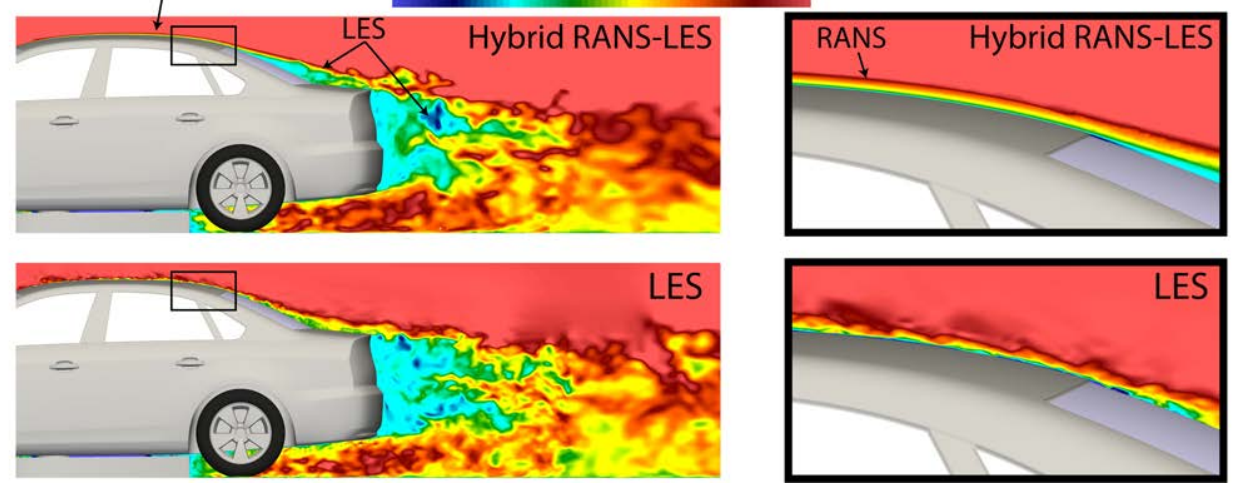

Figure 9: Illustration of the rationale of hybrid RANS-LES turbulence models (a), where the near-wall is modeled with a RANS turbulence model (green region) or wall modeled LES (WMLES) (blue region). In the turbulent wake behind the vehicle (yellow region), where the turbulent structures have "detached" from the near-wall region, LES is used to resolve the turbulence. At the bottom, a comparison of the instantaneous total pressure coefficient for a hybrid RANS-LES (b) and LES (c) simulations of the flow over the rear part of the vehicle is seen. In the hybrid RANS-LES simulation (SBES), the near-wall flow is modeled with RANS and behaves steady, while the separated flow is resolved and unsteady. In the LES simulation, the near-wall region is also resolved and thereby unsteady. 


\section{Delayed Detached Eddy Simulation}

The DDES model is based on the original DES model proposed by Spalart et al. [59]. The DES model intends to use RANS for attached flow regions and switch to LES in detached regions away from the wall, the green and the yellow region is Figure 9, respectively. The original DES model is accurate for flow with thin boundary layers and massive separations, which for it was originally designed. However, it struggles in flows with thick boundary layers and shallow separations [60]. This is due to the mesh size parallel to the wall being smaller than the thickness of the boundary layer, causing the DES model to switch to LES too early. This results in under-resolved Reynolds stresses and thereby too low eddy viscosity and skin friction, which often leads to premature separation if the grid is too coarse to maintain LES, known as Grid Induced Separation (GIS).

To solve the GIS, an improved version was published in 2006 by Spalart et al. [60], called Delayed DES (DDES). The DDES model utilizes a stronger shielding of the RANS region in order to be significantly less sensitive to GIS. This is achieved by making the DDES model include a boundary layer sensor and a shielding function, $f_{d}$, resulting in a modification of the DES model length scale formulation, $L_{D D E S}$, Equation 43.

$$
L_{D D E S}=L_{R A N S}-f_{d} \max \left(0, L_{R A N S}-C_{D D E S} \Delta_{\max }\right)
$$

Here $L_{R A N S}$ is the RANS model length scale, as defined by the specific RANS model, $C_{D D E S}$ is an empirical constant (with the typical value of 0.65 ) and $\Delta_{\max }$ is the maximum edge length of the cell. This is meant to ensure that the switch from RANS to LES occurs outside the boundary layer $[60,34]$.

\section{Improved Delayed Detached Eddy Simulation}

The IDDES model is meant as an improvement of the DDES model, as it includes wall model possibilities for LES in order to broaden the application areas for the model [61]. The model is designed to be similar to the DDES model with the possibility to switch to wall model LES (WMLES) if enough upstream unsteadiness occurs, e.g., blue region in Figure 9. This results in resolved turbulent structures inside in the near-wall region, which otherwise eventually would be suppressed by the RANS model. To achieve the switching the WMLES length scale, $L_{W M L E S}$, needs to be included into the formulation of the IDDES model length scale [34], Equation 44.

$$
L_{I D D E S}=\tilde{f}_{d}\left(1+f_{\text {restore }}\right) L_{R A N S}+\left(1-\tilde{f}_{d}\right) L_{L E S}
$$

Here $\widetilde{f}_{d}$ is the DDES RANS-LES blending function for the IDDES model, and $L_{L E S}$ is the LES length scale. A boosting function $f_{\text {restore }}$ is used to correct the reduction of RANS modeled stresses near the LES-WMLES interface, as the eddy viscosity is lowered by the LES model [34]. 


\section{Important Factors for Accurate Scale-Resolving Simulations of Automotive Aerodynamics}

\section{Shielded Detached Eddy Simulation and Stress Blended Eddy Simulation}

In recent times some more advanced and improved versions of the DES methodology have arisen, where one of the features is to generate faster transition between the RANS and LES regions. In the DDES model the LES grid length scale is defined as $\Delta=\Delta_{\max }$, Equation 43. This can be problematic, as it may result in too high eddy viscosity in separating shear layers, as an effect of the mesh aspect ratio. This leads to a slow transition from the RANS to LES region, and so-called gray-area, where the initial lack of resolved turbulence results in a region which neither is RANS nor LES. This leads to a sort of pseudo-laminarturbulent transition to occur before fully developed resolved turbulence is achieved further downstream [34].

The SDES turbulence model is an improved version of the DES model concept, developed by Menter $[62,63]$, which is designed to have a much stronger RANS shielding function and a faster transition from the RANS to LES region to minimize the gray-area between the regions. The stronger shielding function ensures that the RANS modeling in the nearwall region is intact, even during severe mesh refinement where the DDES model can fail [63]. The formulation of the SDES shielding function, $f_{S D E S}$, is, unfortunately, ANSYS proprietary and is hence not openly available for the public. However, it is important to note, that the SDES model is per se, not a new model, it just has a more efficient RANS region shielding than the DDES model. The faster transition in SDES is achieved by an improved definition of the grid length scale, Equation 45.

$$
\Delta_{S D E S}=\max \left(\sqrt[3]{V}, 0.2 \Delta_{\max }\right)
$$

Here $V$ is the cell volume, while the second part ensures a viable limit for cells with high aspect ratios. This, together with a lower $C_{S D E S}$ model constant $\left(C_{S D E S}=0.4\right.$, which is calibrated for shear layers), results in much lower eddy viscosity in the early onset of the LES region and faster generation of resolved turbulent structures, compared to the DDES model. It is important to note that this grid length scale can easily be introduced in the DDES model, but would result in much weaker RANS shielding and higher risk of GIS.

An extension of the SDES model is the SBES model, developed by Menter [62], which is based on the SDES model but with an addition of blending the underlying RANS model directly to any existing algebraic LES model. The blending of the turbulence stress tensor is defined as

$$
\tau_{i j}^{S B E S}=f_{S D E S} \tau_{i j}^{R A N S}+\left(1-f_{S D E S}\right) \tau_{i j}^{L E S}
$$

where $\tau_{i j}^{R A N S}$ and $\tau_{i j}^{L E S}$ are the stress tensor from the RANS model and the LES SGS model, respectively. The same formulation is used to define the SBES model eddy viscosity, Equation 47.

$$
\nu_{\tau}^{S B E S}=f_{S D E S} \nu_{t}^{R A N S}+\left(1-f_{S D E S}\right) \nu_{S G S}
$$

Here $\nu_{\tau}^{S B E S}$ is the eddy viscosity for the SBES model and $\nu_{\tau}^{R A N S}$ from the RANS model. 


\subsection{Domain and Boundary Conditions}

Two types of computational domains are used in this thesis, open-road (Paper III) and replicate of the experimental measurements setting (Paper I, II, IV-VI), Figure 10. Common for the domains is that they follow the SAE Standard for CFD simulations for aerodynamics of heavy and commercial vehicles [64], which is that the domain inlet and outlet should be located 3-5 and 5-8 vehicle lengths $(L)$ from the test-object, respectively. The side and top boundaries are positioned, so the cross-sectional area of the domains for open-road simulations result in a solid blockage of less than $2 \%$. This ensures neglectable effects from the boundary conditions on the flow field around the vehicle, Figure 10 panel a. For the domains which replicates experimental conditions, the cross-sectional area is determined by the used experimental conditions, where the maximum solid blockage reaches $6.4 \%$ for the DrivAer model at $7^{\circ}$ yaw. However, for Paper V and VI, compensation of forces and pressure are made for the effects of the solid blockage.

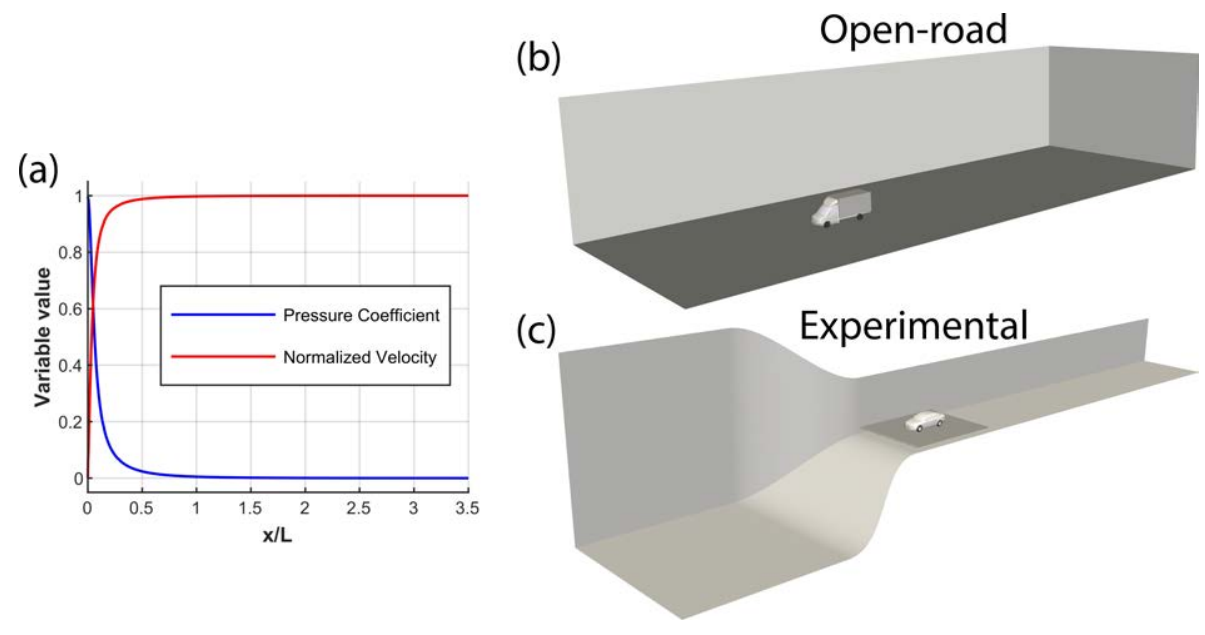

Figure 10: (a) Effect on the velocity and pressure upstream a vehicle to the domain inlet. Neglectable effects on velocity and pressure occur already at 2 vehicle lengths upstream of the vehicle. In (b) and (c), the open-road domain used in Paper III and experimental conditions used in Paper V and VI are seen, respectively.

The inflow into the domain is modeled with a uniform velocity profile, with turbulent values matching the experimental test-section when available, while the outflow of the domain is modeled with zero-gauge pressure-outlet. For the simulations, including RANS turbulence modeling (Paper I and III-VI), turbulence intensity of 0.01 to $0.1 \%$, together with a turbulent viscosity ratio of 50-200, is used. In Paper V and VI, the inlet turbulence is tuned so similar turbulence intensity as seen in the measurements $[65,66]$ is achieved in the test-section. For the LES simulation (Paper II), turbulence is generated with a spectral synthesizer and tuned such a similar turbulent intensity, as in the measurements $[67,68]$, is obtained in the test-section. 


\section{Important Factors for Accurate Scale-Resolving Simulations of Automotive Aerodynamics}

The surfaces of the vehicles and the solid surfaces of the experimental domain are modeled as no-slip walls. To model the rotating wheels and moving ground in Paper III, a wall velocity is applied to the rotating surfaces and ground. In the Paper III and IV, the side and top domain boundaries are modeled as free-slip walls or with symmetry conditions. This differs from the other investigations where the experimental settings are modeled as no-slip walls to get the correct boundary layer build up inside the test-section, thereby slightly affecting the solid blockage effect. For the steady-state RANS simulations of symmetric bodies and symmetric inflow conditions, Paper I and III, only half the domain is simulated with symmetry conditions.

In Paper II, V and VI, some of the essential components of the test-section are included in order to establish a similar flow field, as in the experiments, around the vehicle bodies. This included the airfoil stilts supporting the bodies, splitter plates and the balance, Figure 11.
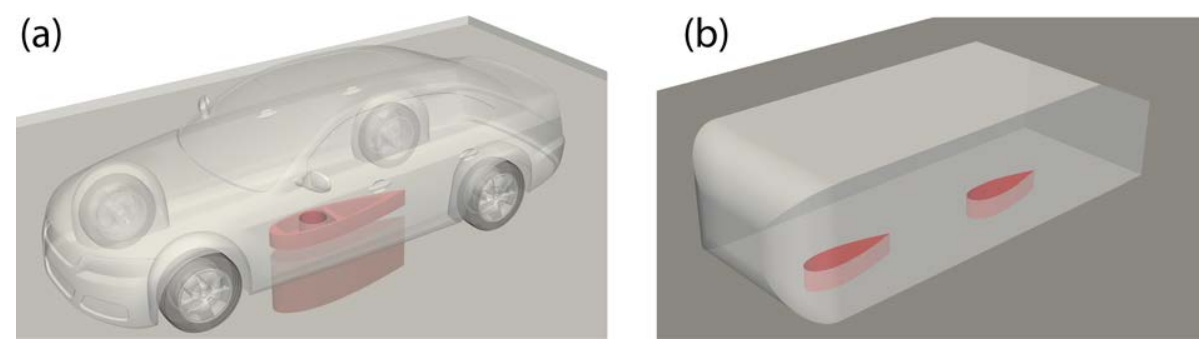

Figure 11: Close up on the vehicle set-up in the test section for replication of the measurement conditions. Note that both the DrivAer (a) and Ahmed (b) vehicle bodies are supported by symmetric airfoil stilts (red).

\subsection{Mesh}

To minimize the numerical diffusion and number of cells, and thereby the cost of the simulations, hexahedral cell-based meshes are used in this thesis [19]. Two types of mesh strategies have been applied to fulfill hexahedral dominated meshes: blocked structured hexahedral meshing (Figure 12) and Cartesian hybrid meshing (Figure 13). Similar for both meshing techniques is that mesh refinement is focused adjacent to the vehicle surfaces and in regions containing strong gradients (e.g., separations and stagnation zones). Polyhedral meshes have also been considered, but as it is difficult to achieve satisfactory quality for thin prisms cells around sharp edges, it has not been used in any publication.

Two different near-wall meshing approaches have been applied, the $y^{+} \leq 1$ and $y^{+} \approx 30$ approaches, where the latter utilize wall functions. For both approaches, at least 10 cells cover a well-developed boundary layer, and no element size change over 1.2 existed within the near-wall region, to ensure well-resolved gradients. For the SA, SST and hybrid RANSLES turbulence models the first cells are placed within the viscous sublayer, to achieve $y^{+}$ values below unity (Figure 13 panel b), while for the realizable $k-\varepsilon$ model it is positioned 
in the log-law layer $\left(y^{+}=30\right)$. Tests were performed with the realizable $k-\varepsilon$ EWT model on the $y^{+}<1$ mesh but did not results in any significant differences (Paper III). The used mesh strategy, near-wall modeling approach, and the amount of cells in the mesh is presented for each appended paper and used turbulence modeling approach in Tabel 1.

Table 1: Details of meshes used in the appended papers and this thesis based on the used turbulence model and near-wall modeling approaches. Mesh sizes are for full vehicle domains.

\begin{tabular}{lcccr} 
Paper & $\begin{array}{c}\text { Turbulence } \\
\text { model }\end{array}$ & Type of mesh & \# of cells & $y^{+}$approach \\
\hline I & RANS & Hexahedral & $11.4 \cdot 10^{6}$ & $1 \& 30$ \\
I & RANS-LES & Hexahedral & $90 \cdot 10^{6}$ & 1 \\
II & LES & Hexahedral & $33.8 \cdot 10^{6}$ & 1 \\
III & RANS & Hybrid & $166 \cdot 10^{6}$ & 30 \\
III & RANS-LES & Hybrid & $382 \cdot 10^{6}$ & 1 \\
IV & RANS-LES & Hybrid & $56 \cdot 10^{6}$ & 1 \\
V & RANS-LES & Hybrid & $102 \cdot 10^{6}$ & 1 \\
VI & RANS-LES & Hybrid & $102 \cdot 10^{6}$ & 1 \\
\hline
\end{tabular}

\section{Hexahedral Mesh}

For the two geometrically simple bodies, Paper I and II, the mesh only consisted of hexahedral cells, and are created in ANSYS ICEM CFD (version 15.0). The meshes are created from rectangular blocks, and the vehicle models are enclosed by two O-grids for efficient distribution of the cells, Figure 12 .

The hexahedral mesh used in the LES simulations of the Ahmed body (Paper II), Figure 12 , consists of very fine mesh resolution in the near-wall region in order to resolve the small turbulent structures. The first node is positioned within the viscous sublayer to achieve $y^{+}<1$, while the spanwise and streamwise cell size is no more than a factor 14 larger than its wall-normal size, to fulfill LES mesh resolution recommendations, presented in section 2.5. No element size change over 1.15 exist in the vicinity of the body, while up to 1.3 exist in the non-disturbed free-stream region. 


\section{Important Factors for Accurate Scale-Resolving Simulations of Automotive Aerodynamics}

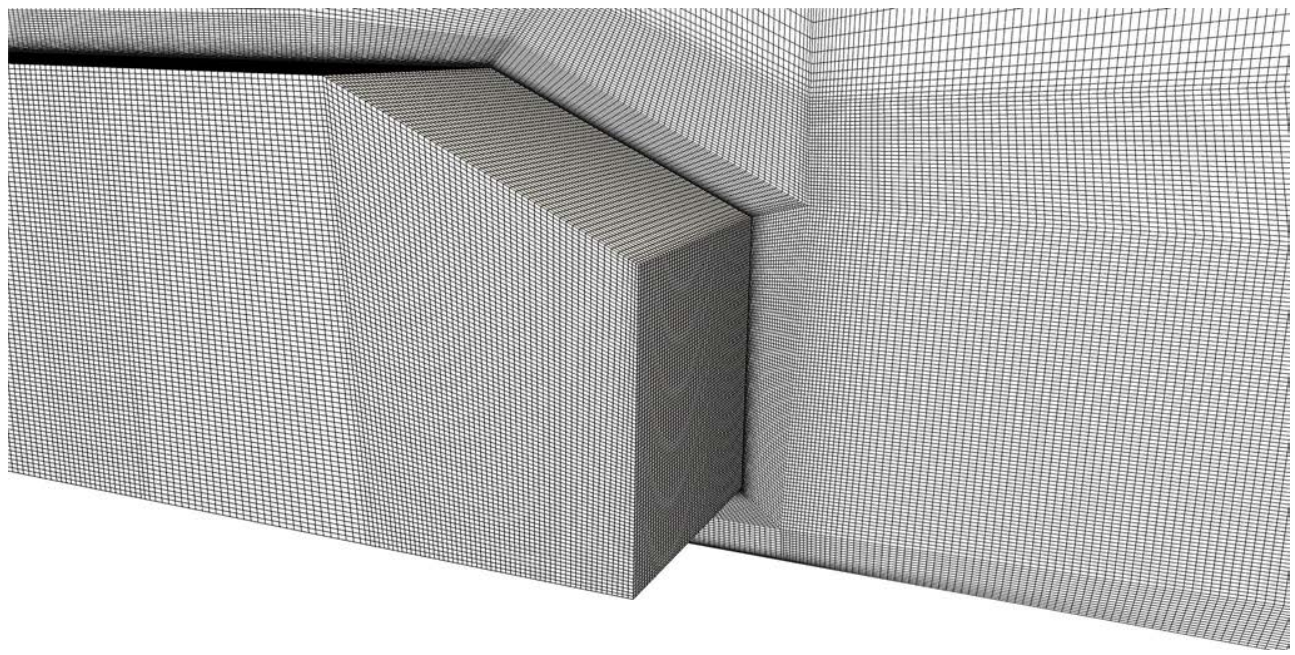

Figure 12: Hexahedral block mesh for the rear of the Ahmed body. Note the O-grid block structure in the vicinity of the body to obtain a high spatial resolution in the near-wall region and efficient spacing of the cells in the rest of the domain.

\section{Cartesian Hybrid Mesh}

The Cartesian hybrid mesh is used for the geometrically advanced vehicles, Paper III-VI, and are created in two steps. The surface meshes are created in ANSA (BETA CAE Systems S.A. Thessaloniki, Greece) and the volume meshes are created in ANSYS Fluent (versions 15.0 to 2019R1). The surface mesh consists of triangular surface elements, with a surface element skewness of less than 0.5. An exception is the surface mesh in Paper III, which also consists of quad surface elements, to reduce the number of surface elements on large flat surfaces. From the surface mesh, prisms layers are created. At a minimum, 10 layers are used on the vehicle surface for the RANS simulations in Paper III, while up to 20 layers are used on the vehicle surfaces for the hybrid RANS-LES simulations, Figure 13, to ensure sufficient resolution of the gradients in the near-wall region. The prisms layers are created by specifying the first cell thickness and the aspect ratio of the last cell, enabling a smooth transition to the bulk mesh and control of the $y^{+}$value. The prisms cells are connected to the Cartesian grid with a thin layer of tetrahedral and pyramid elements. The maximum allowed skewness (equilateral volume deviation skewness) of the volume meshes is 0.95 . 


\section{(a)}

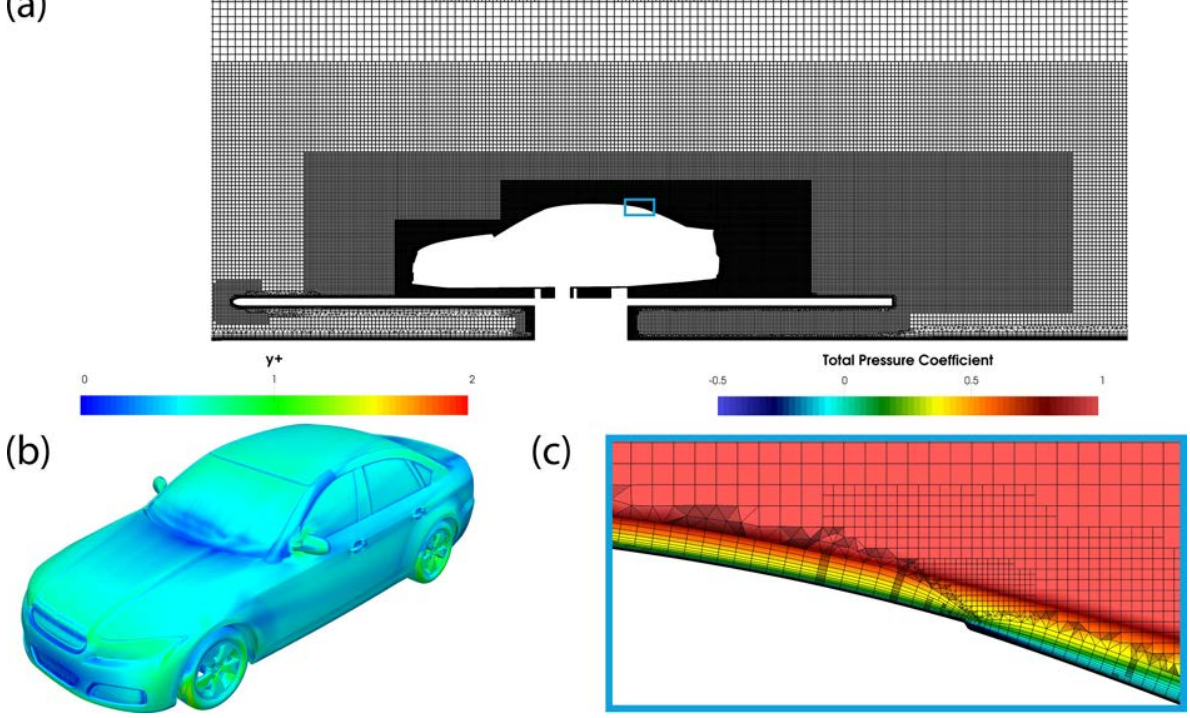

Figure 13: Cartesian hybrid mesh for the DrivAer car body in the wind tunnel setting. (a) overview of the mesh refinement regions for capturing the strong gradients. (b) DrivAer car body surface colored with the time-averaged $y^{+}$value. (c) zoom in on the near-wall mesh resolution at the rear part of the roof, where it can be seen that the prisms layers cover the boundary layer.

\section{Mesh Sensitivity Analysis}

To ensure that the results and findings are not sensitive to the mesh resolution, the mesh resolution has been investigated for each vehicle body. The commonly used method for all appended papers is to ensure convergence of the aerodynamic forces with increased mesh resolution, especially for $C_{D}$ and $C_{L}$, both for the whole and individual parts of the vehicle.

For hybrid RANS-LES methods, this is trickier, as the turbulence models formulation can lead to a different behavior of the turbulence model during mesh refinement. This is especially true for the SAS and IDDES models. The SAS model is formulated to switch from steady RANS mode to unsteady "LES-like" mode when enough unsteadiness is detected. With increasing mesh resolution, this can lead to more and more LES behavior, which differs from the RANS modeling as it resolves turbulent structures and therefore, can capture the anisotropic turbulence behavior, leading to changing behavior of the turbulence model. With infinite mesh refinement, a convergence of the investigated parameters would most likely occur. However, before sufficient mesh convergence is achieved, the computational cost can be too high for the initial purpose of the model. Similar behavior can occur for DES-based models, as the RANS modeling for the near-wall switches to LES, even though the mesh still is not fine enough for wall-resolved LES, possibly leading to GIS. For this reason, the mesh sensitivity study for the hybrid RANS-LES simulation of the DrivAer 


\section{Important Factors for Accurate Scale-Resolving Simulations of Automotive Aerodynamics}

car body is done with the SBES model, which ensures shielding of the RANS region for even LES like meshes [62].

For the LES simulations of the Ahmed body, not only the forces are investigated during the mesh sensitivity analysis. Mesh resolution investigations for LES can be trickier than for RANS, or even DNS, as the partial differential equations are dependent on the grid spacing [69]. Depending on the mesh resolution, different amounts of the energy spectrum for the turbulence will be resolved and modeled, Equation 8. This affects the solution both locally and globally, as locally under-resolved turbulence might affect the global solution. Therefore, also the mesh resolution is investigated for the LES ability to resolve turbulent structures in the near-wall and separated regions. The near-wall mesh resolution is investigated for the spanwise, $l^{+}$, and streamwise, $s^{+}$, non-dimensional wall units. Here $l^{+}=\tau \Delta l / \nu$ and $s^{+}=\tau \Delta s / \nu$, where $\Delta s$ and $\Delta l$ are the spanwise and streamwise cell sizes, respectively. The used mesh resulted in $l^{+}<20$ and $s^{+}<40$, which follows LES grid resolution recommendations for turbulent channel flow [70].

The separated flow regions are investigated in terms of how much of the turbulent kinetic energy is resolved, $M(x, t)$, and the normalized two-point correlation, $B_{i i}^{n o r m}$. According to [71], a well-resolved LES should resolve at least $80 \%$ of the turbulent kinetic energy. The percentual amount resolved turbulent kinetic energy can be defined as

$$
M(x, t)=\frac{k_{t o t}(x, t)-k_{s g s}(x, t)}{k_{t o t}(x, t)}
$$

where $k_{t o t}(x, t)$ is the total turbulent kinetic energy and $k_{s g s}(x, t)$ is the SGS modelled part. The modeled SGS turbulent kinetic energy can be approximated as

$$
k_{s g s}(x, t)=\frac{\nu_{t}(x, t)^{2}}{\left(\Delta(x, t) \cdot C_{s}(x, t)^{2}\right)}
$$

where $\nu_{s g s}$ is the SGS eddy viscosity, $\Delta$ the filter width and $C_{s}$ is the Smagorinsky model constant. In the LES simulations, it is seen that the majority of the cells resolve more than $80 \%$ of the total turbulent kinetic energy, Figure 14 panel a, thereby following the resolution recommendations [71].
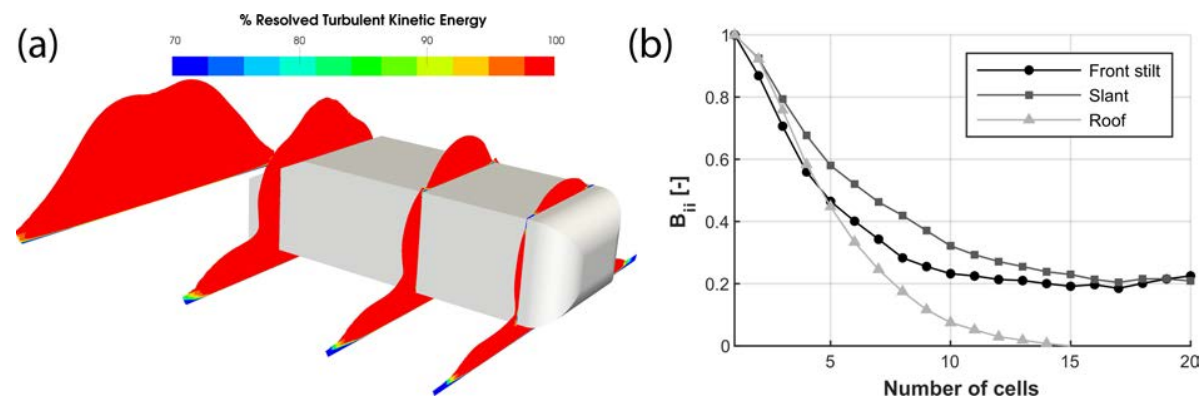

Figure 14: (a) Percentual amount resolved turbulent kinetic energy of the total (resolved + modeled) turbulent kinetic energy, seen in four planes around the Ahmed body. (b) Two-point correlation for three regions around the Ahmed body. 
The normalized two-point correlation indicates how many cells cover the largest eddies of the flow, Equation 50.

$$
B_{i i}^{n o r m}=\frac{1}{v_{i}, r m s\left(x_{i}^{A}\right) v_{i}, r m s\left(x_{i}^{A}+\widehat{x}_{i}\right)} \overline{v_{i}^{\prime}\left(x_{i}^{A}\right) v_{i}^{\prime}\left(x_{i}^{A}+\widehat{x}_{i}\right)}
$$

Here $x_{i}^{A}$ denotes the starting point, $\widehat{x}_{i}$ the distance from the starting point $\mathrm{A}, v_{i}$ the velocity component and $v_{i}^{\prime}$ the fluctuating part of the velocity component. This is investigated in three turbulent regions of the Ahmed body, and a signal correlation of 0.2 or higher is observed for more than 14 cells downstream the front stake and at the slant, Figure 14, which is well within the recommendations of at least eight cells $[72,73]$. Slightly lower correlation is seen at the top part of the front of the body, where the same level of correlation is seen for only seven cells.

\subsection{Solver Settings}

The cell-centered finite volume solver ANSYS Fluent (versions 15.0 to 2019R1) is used for all simulations. The used solver settings can be divided into two categories, based on if RANS or SRS turbulence modeling is used, as presented in Table 2.

The coupled pressure-velocity scheme is used for the RANS simulations, as it is robust and efficient for steady-state simulations [63], compared to segregated pressure-velocity schemes. However, for SRS simulations, the Semi-Implicit Method for Pressure linked Equations-Consistent (SIMPLEC) pressure-velocity scheme is mainly used, as it is computationally faster per iteration than the coupled scheme. This is important as several inner-loop iterations are needed for each time-step, even though the coupled solver might require fewer iterations. The spatial discretization for the gradients is solved by using the Least Squares cell-based method, which maintains $2^{\text {nd }}$ order accuracy at a low cost. Both the standard and $2^{\text {nd }}$ central difference pressure interpolation schemes have been used for the RANS simulations. Performed tests show small differences between the schemes for steady-state simulations, particularly for fine meshes, but the standard scheme sometime provides improved convergence of the solution. For SRS simulations, the $2^{\text {nd }}$ order pressure scheme is used, as strong local and time-dependent gradients can occur during the solution.

The momentum discretization follows the best practice guides for RANS and SRS simulations [45]. The spatial discretization of the convective terms in the momentum equations are calculated by use of the $2^{\text {nd }}$ order upwind for the RANS simulations. For SRS simulations, the Bounded Central Differencing scheme is used, as it enables low numerical diffusion by using the central differencing scheme but still ensures stability by blending first and second-order upwind scheme when needed. The central difference scheme was tested for LES, but caused unphysical instabilities and is therefore not used. The central differencing scheme is used for the spatial discretization of the diffusion terms.

For the turbulence equations $(k, \varepsilon, \omega, \tilde{\nu})$ the $1^{\text {st }}$ and $2^{\text {nd }}$ order upwind schemes are used. Most often, no or small differences are seen between the $1^{\text {st }}$ and $2^{\text {nd }}$ scheme order of 


\section{Important Factors for Accurate Scale-Resolving Simulations of Automotive Aerodynamics}

(a)

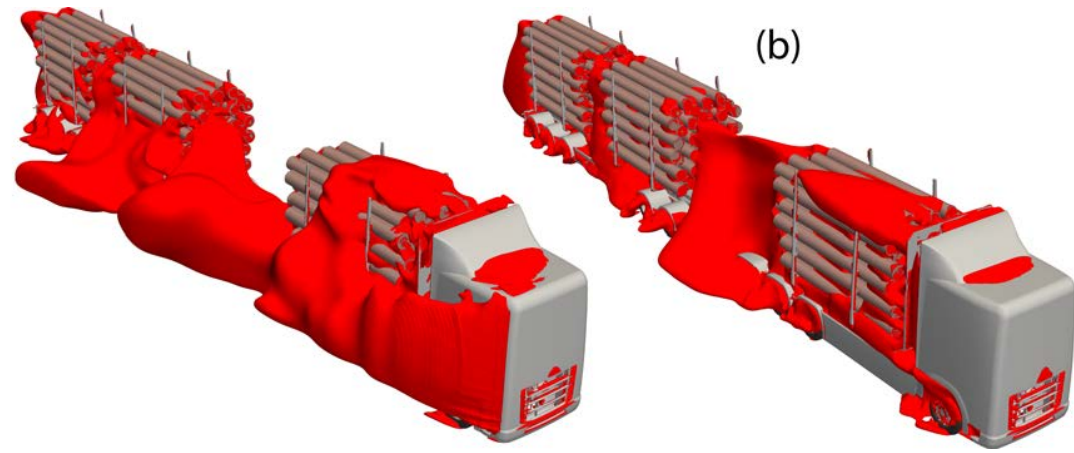

Figure 15: $1^{\text {st }}(\mathrm{a})$ and $2^{\text {nd }}(\mathrm{b})$ order upwind scheme for the $k$ and $\varepsilon$ equations for a RANS simulation of a timber truck at $7.5^{\circ}$ yaw. The red surface is zero $x$-velocity and indicates separation. Much larger separation is seen around the leeward A-pillar, which affects the downstream flow field significantly when using $1^{\text {st }}$ order upwind scheme for the turbulence equations.

the turbulence equations in RANS simulations. However, it has been seen that the flow around the A-pillar of trucks can be rather sensitive to this, as $1^{\text {st }}$ order can result in a too diffusive solution, lowering the level of turbulent kinetic energy and resulting in too early separation, Figure 15 . The temporal resolution is solved with the $2^{\text {nd }}$ order bounded implicit time integration for all unsteady simulations.

Table 2: Numerical set-ups based on the turbulence modelling method.

\begin{tabular}{lcc} 
& RANS & SRS \\
\hline$p-v$ & Coupled & SIMPLEC \\
Spatial gradients & Least square cell based & Least square cell based \\
$\begin{array}{l}\text { Pressure } \\
\text { Momentum }\end{array}$ & Standard, $2^{\text {nd }}$ order & $2^{\text {nd }}$ order \\
& $2^{\text {nd }}$ order upwind & Bounded Central \\
Turbulence equations & $1^{\text {st }}, 2^{\text {nd }}$ order upwind & $1^{\text {st }}, 2^{\text {nd }}$ order upwind \\
Temporal & - & $2^{\text {nd }}$ order bounded implicit \\
\hline
\end{tabular}

The steady-state RANS simulations run until the solution normalized residuals at least reach below $10^{-4}$, and a stabilized state for lift and drag exist. The solution then run for another 500 iterations for averaging, as small fluctuations can exist in RANS simulations of bluff bodies.

The unsteady simulations are initialized from steady-state RANS simulations and turned unsteady with a vortex synthesizer. Several inner loop iterations are needed for every time step to ensure that the normalized residuals fall below $10^{-5}$ for the instantaneous solution. A larger time-step size $(\approx 5 \cdot \Delta t)$ is then used to speed-up the elimination of the initialization effects before the small time-step size is used. Several convective flow units $\left(t=L / U_{\infty}\right)$ are run before time-averaging to ensure the solution has reached a stabilized state, which mainly is monitored for the forces. Time-averaging is then done until only 
small fluctuations (typically $\Delta C_{D}=0.001$ ) existed for the time-averaged forces. In Figure 16 , the overall simulation procedure for the unsteady simulations is seen.

The sensitivity of the time-step size is investigated in Paper V and showed that much larger time-steps can be used and still achieve high accuracy in the simulations. In Table 3, the used time-step sizes for SRS are presented together with the normalized time-step size (indicating the number of time-steps needed for convective flow units) and the number of cells in the domain which are within the Courant-Friedrichs-Lewy condition of less than unity $(C F L \leq 1)$.

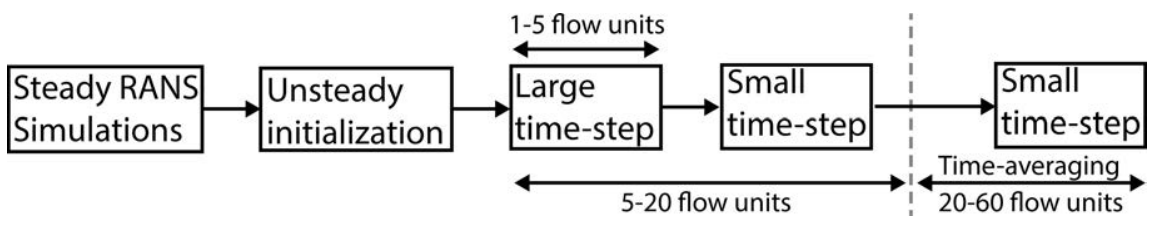

Figure 16: Unsteady simulation procedure. The unsteady simulation is initialized from a steadystate RANS simulation and then turned into an unsteady field with a vortex synthesizer. Larger time-steps are used in the beginning for faster removal possible initialization effects before switching to the time-step used for the analysis.

Table 3: Temporal resolution used in this thesis for SRS. The third and fourth columns indicate the number of time-steps needed for a convective flow unit and how many cells in the domain that are within the $C F L \leq 1$ criterion.

\begin{tabular}{lccr} 
Paper & $\Delta t[\mathrm{~s}]$ & $\left(L /\left(\Delta t \cdot U_{\infty}\right)\right.$ & Cells CFL $\leq 1$ \\
\hline I & $2 \cdot 10^{-5}$ & 2137.5 & $99 \%$ \\
II & $3.2 \cdot 10^{-5}$ & 1561 & $100 \%$ \\
III & $2.9 \cdot 10^{-5}$ & 45067 & $99.9 \%$ \\
IV & $10^{-5}$ & 4885 & $99.9 \%$ \\
V $(\Delta t \min )$ & $1.4 \cdot 10^{-6}$ & 20850 & $99.99 \%$ \\
V $(\Delta t \max )$ & $1.4 \cdot 10^{-4}$ & 208.5 & $61 \%$ \\
VI & $2.8 \cdot 10^{-5}$ & 1042.5 & $88 \%$ \\
\hline
\end{tabular}

Table 3 highlights that the used time-step sizes for the unsteady simulations in Paper I-IV are well resolved in time, as more than $99 \%$ of the cells are within the $C F L \leq 1$ criterion.

\subsection{Experimental Data}

In order to see how accurate the simulations are, comparison to experimental data or very accurate CFD simulation is needed. As mentioned before, DNS includes no modeling of the turbulence and can be seen as very accurate (if done properly). However, the present level of DNS is nowhere near realistic Reynolds numbers and geometries used in 


\section{Important Factors for Accurate Scale-Resolving Simulations of Automotive Aerodynamics}

automotive aerodynamics, and will most likely not be soon [59]. Therefore, comparisons to experimental measurements are used in this thesis for four of the appended Papers (I, II, V and VI). The data is obtained from both literature and direct collaboration with experimental research groups.

In Paper I, experimental data are taken from literature [23], where wind tunnel measurements have been performed for a simplified truck body. Force and pressure measurement existed together with some descriptions of the wind tunnel test section, only making it possible to see trends and accuracy on an overall level.

In Paper II, experimental data from Particle Image Velocimetry (PIV) performed by a research group in Monash University, Australia, are compared with LES. The flow measurements of the Ahmed body were performed in the water tunnel at Fluids Laboratory for Aeronautical and Industrial Research (FLAIR), which is a free-surface closed-loop water tunnel. The Ahmed body was supported on a splitter plate with two symmetrical airfoils, Figure 11 panel b. The high spatial resolution PIV consisted of several planes with a resolution of $0.006 W$ and $0.003 H$, where $W$ and $H$ are the width and height of the Ahmed body used in the measurements, respectively [67, 68]. This high spatial resolution data made it possible to accurately compare the vortical structures arising from the C-pillars and wake of the body and even some of the behavior of the near-wall flow. The data was received in raw format, and the post-processing is made in Matlab, where the vorticity and vortex center are attained. The vortex center is calculated with the $\Gamma_{1}$ function, which finds the rotation center of a region of interest and is described in [74] and Paper II.

For the studies of the DrivAer car body, wind tunnel measurements performed in the closed-loop GroWiKa wind tunnel (illustrated in Figure 10 panel c) at Technische Universität Berlin (TU Berlin) are compared with CFD simulations. No moving ground or rotating wheels were available; hence the model hangs $2 \mathrm{~mm}$ above a splitter plate and is supported by the balance, which is covered with a symmetrical airfoil, Figure 11 panel a. The data consisted of forces (drag and lift), pressure measurements, oil film visualizations, and flow field measurements of the rear wake by use of PIV. Raw images of the oil film visualization and raw pressure measurement data were obtained directly from TU Berlin, while the forces and PIV measurements are acquired from published papers [75, 65, 66, 76].

An important factor to consider for the experimental measurements is the purpose of the measurements. Are the measurements purely done for aerodynamic development, where the overall forces and trends are of interest or are they done for detailed analysis of the flow field and validation? In this thesis, both types of measurements are included, as the measurements used in Paper I only includes comparisons to forces, while detailed comparisons of the complex flow downstream the Ahmed body are performed in Paper II. The experimental measurements of the DrivAer car body are in the middle ground of these two categories. This is important to consider, as too in-depth analysis and comparison of the flow field to pure aerodynamic development measurements may be misleading, due to the accuracy of the measurements and experimental set-up. However, significant trends in the flow field and forces are less sensitive to possible deceptions and can, therefore, be used. 


\subsection{Flow Descriptors}

Flow can be described and quantified in many ways. The following are some measures commonly used to describe the flow and surface field in automotive aerodynamics. For easier comparison, most of the flow variables are presented as non-dimensional coefficients. Pressure is the dominating component of the aerodynamic forces for bluff bodies. The static pressure coefficient is defined as

$$
C_{P}=\frac{p-p_{\infty}}{\left(0.5 \rho_{\infty} U_{\infty}^{2}\right)}
$$

where $p$ is the static pressure and $p_{\infty}$ the atmospheric pressure or as in Papers (V and VI) the reference pressure in the test-section of the wind tunnel. The denominator is also known as the free-stream dynamic pressure, $q_{\infty}$. Skin friction can be important to visualize where the flow separates and patterns of the near-wall flow. The skin friction coefficient is defined as

$$
C_{f}=\frac{\tau}{q_{\infty}}
$$

where $\tau$ is the wall shear stress. The total pressure is the sum of the static and dynamic pressure and presents the sum of the potential (pressure energy) and kinetic energy per volume unit, respectively. The total pressure coefficient is therefore useful for understanding the pressure losses of the flow and defined as

$$
C_{p_{\text {tot }}}=\frac{p+q}{q_{\infty}}
$$

For unsteady flow, it can be of interest to investigate the fluctuating part, $\phi^{\prime}$, of the flow. Hence the statistically averaged variation of the fluctuations around the average can be described by the root-mean-square (RMS) value, which is defined as

$$
\phi_{R M S}^{\prime}(x, t)=\sqrt{\overline{\left(\phi^{\prime}(x, t)\right)^{2}}}=\sqrt{\frac{1}{\Delta t} \int_{0}^{\Delta t}(\phi(x, t)-\bar{\phi}(x))^{2} d t}
$$

where the decomposition in Equation 11 has been used.

In turbulent flow, visualization of the Q-criterion can be of interest, to get an understanding of the behavior of the coherent turbulent structures. It is defined as the second variant of the velocity-gradient tensor and defined as

$$
Q=0.5\left(\Omega^{2}-S^{2}\right)
$$

where $S_{i j}$ is the strain rate (Equation 10) and $\Omega$ is the absolute value of vorticity, defined as $\Omega=\sqrt{2 \omega_{i j} \omega_{i j}}$, where

$$
\omega_{i j}=0.5\left(\frac{\partial u_{i}}{\partial x_{j}}-\frac{\partial u_{j}}{\partial x_{i}}\right)
$$

With the Q-criterion, it is possible to visualize vorticity, which characterizing turbulent vortices, but without showing steady shear layers, occurring where $S=\Omega$ [45]. 


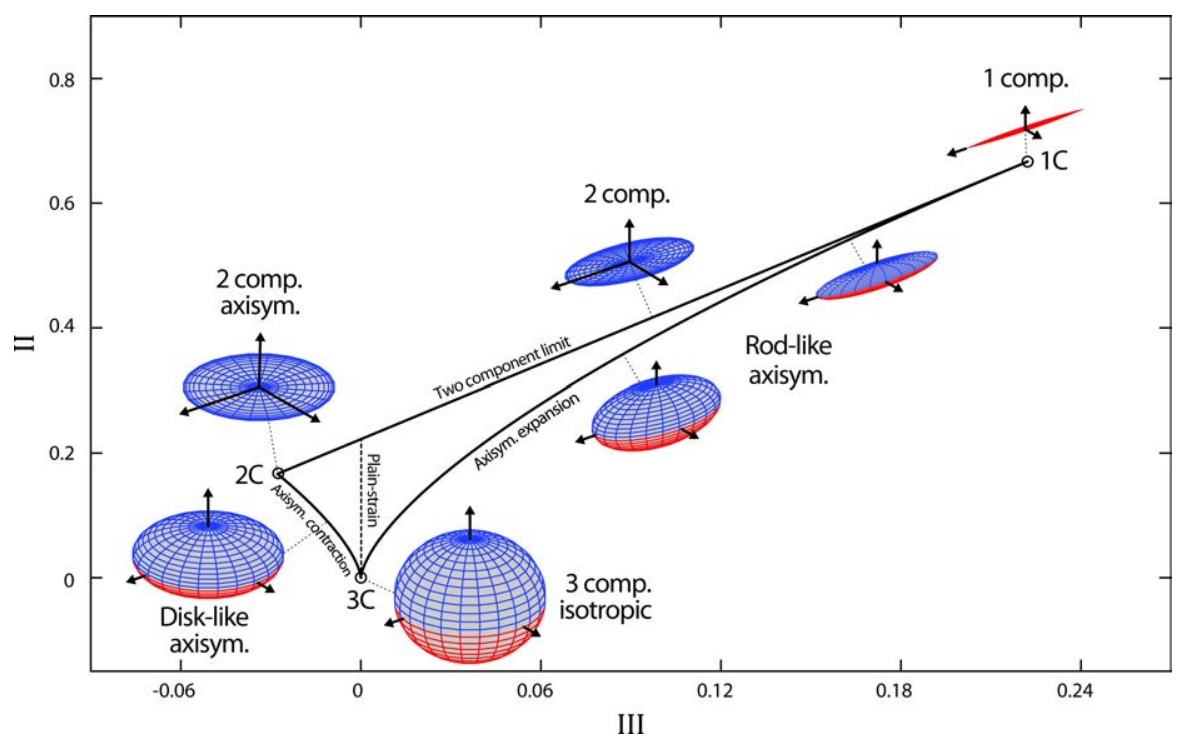

Figure 17: Turbulence states characterized from the anisotropy tensor in invariant coordinates. Within this triangle-like region (called Lumley triangle), all existing physical states of turbulence are found. Three extreme points exist: one-component (1C), two-component $(2 \mathrm{C})$, and three-component $(\mathrm{3C})$ isotropic turbulence, where the fluctuations are limited to the number of components. Reprinted from [77], with permission from Elsevier.

Another useful quantity to describe the unsteady flow behavior is the turbulent kinetic energy $k$ (TKE), as previously described in Equation 17. To evaluate SRS, it can be useful to determine the amount of resolved $k_{\text {res }}$ and modeled $k_{\text {mod }}$ TKE. The normalized resolved TKE is defined as

$$
\frac{k_{r e s}}{U_{\infty}^{2}}=\frac{0.5\left(\overline{u^{\prime 2}}+\overline{v^{\prime 2}}+\overline{w^{\prime 2}}\right)}{U_{\infty}^{2}}
$$

where $u^{\prime}, v^{\prime}$ and $w^{\prime}$ are the fluctuating velocity components in the $x, y$ and $z$ directions, respectively.

From the fluctuating velocity components, the symmetric Reynolds stress tensor can be established, Equation 58.

$$
R_{i j}=\overline{\rho u_{i}^{\prime} u_{j}^{\prime}}
$$

However, for even mildly complex geometries, it is difficult to interpret the distinction of the normal and shear stresses, making it difficult to understand the turbulent behavior. This can be avoided by considering the invariants of $R_{i j}$, which is independent of the coordinate frame. The first invariant, I, is the trace of $R_{i j}$

$$
I=R_{k k}
$$

and is analogous to TKE, Equation 17. By only regarding the traceless deviatoric part of the Reynolds stress tensor, Equation 60, it is also possible to unfold the anisotropic be- 
havior of the stress field from the relation of the second (Equation 61) and third invariants (Equation 62).

$$
\begin{gathered}
b_{i j}=\frac{R_{i j}}{R_{k k}}-\frac{\delta_{i j}}{3} \\
I I=b_{i j} b_{j i} \\
I I I=b_{i j} b_{j k} b_{k i}
\end{gathered}
$$

Lumley et al. [78] proposed the use of the second and third invariants as a coordinate system, resulting in a triangular-like region, known as the Lumley triangle, Figure 17. Within this Lumley triangle, all existing physical states of turbulence can be found, and describe the relative size of the principal normal stresses and hence turbulence componentality. Three extreme states exist: one-component (1C), two-component (2C) and three-component (3C) isotropic turbulence. In one-component turbulence, fluctuations are only present in a single direction. For two-component turbulence, the fluctuations only act in a plane. Three-component turbulence is fully isotropic, and fluctuations act randomly in all directions.

Some of the disadvantages with the Lumley triangle is that it is non-linear and remove the location information of the data, which makes interpretation of the turbulent behavior non-intuitive. This has been overcome by introducing an equivalent but linear representation, called a barycentric map [79], which Emory, et al. [80, 81] introduced a new visualization method for, making it possible to color each componentality states with a red-green-blue (RGB) color map, Figure 18. This makes it possible to color the turbulence componentality in the spatial domain.
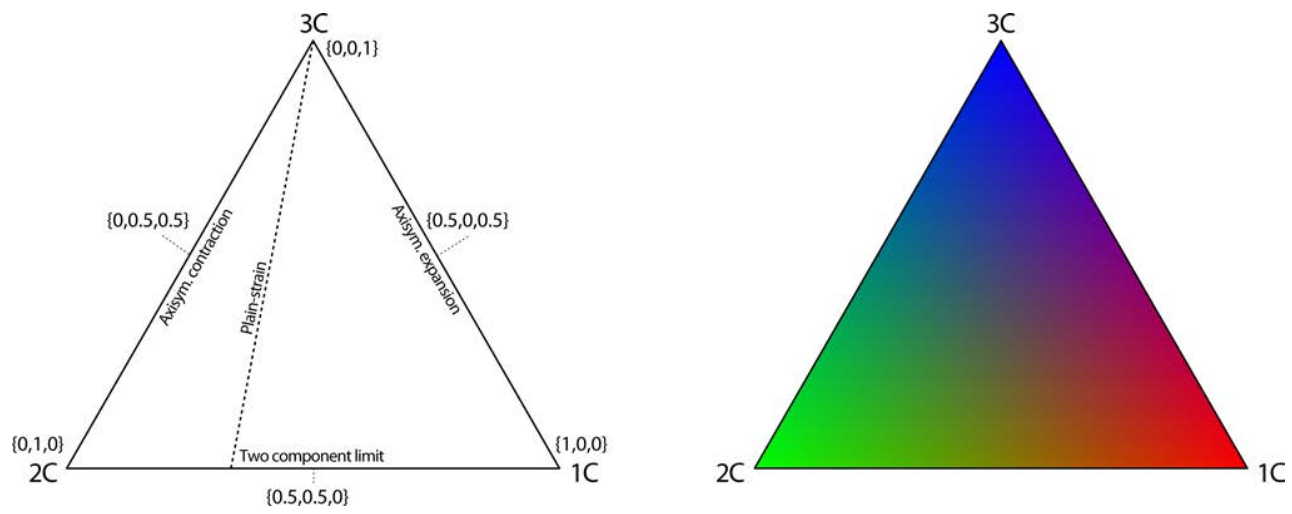

Figure 18: Barycentric colormap of the turbulence componentality, where the local state is governed by anisotropy tensor coordinates. 



\section{Results and Discussion}

\subsection{RANS for Simple Geometries}

At the beginning of the 2010-decade, automotive manufacturers started to consider SRS to achieve higher accuracy for aerodynamic simulations [82]. Before, and even still, many automotive manufactures mainly rely on steady-state RANS simulations, as it offers reasonable accuracy at reasonably low computational cost. One of the main problems with using RANS models for predicting the aerodynamic flow around vehicles is its unreliability to achieve results of high accuracy when compared to measurements consistently. This is investigated in Paper I, where several different RANS and URANS models are evaluated and compared to wind tunnel measurements from [23] for three different geometrical configurations of the Allan body, including both the sharp and rounded front together with two gap distances, Figure 19.

At the time of the experiments (1981), the sharp front was a reasonable representation of the current existing cab shapes, while the rounded version seemed unlikely due to its large radii, and was only considered as a possible shape for future trucks [23]. Today many cabs have large round a-pillars with a radius similar to the rounded version of the Allan body, corresponding to $200 \mathrm{~mm}$ for a full-scale truck. The sharp front is less representative of today's trucks and hence of less priority in this study, and therefore only investigated for a single gap distance, while two gap distances are investigated for the rounded front.

Two different near-wall mesh approaches are used in this study to follow the best practice for each turbulence model. The $y^{+} \approx 30$ approach is only used for the realizable $k-\varepsilon$ model simulations, while $y^{+} \leq 1$ is used for the other models. By the first look (Figure 19), an overall mixed and non-reliable accurate behavior is seen for all investigated models for the different geometrical configurations. Typically, some models are only close to the measurement for one gap distance or front radii, but far off for the other. For the sharp front, surprisingly, the SA model is closest to the measurements, even though it is mainly derived for attached boundary layers, and not separated flows. With the sharp edges, the location of separation is clearly defined by the geometry, which explains the similarly predicted drag for the other models. 


\section{Important Factors for Accurate Scale-Resolving Simulations of Automotive Aerodynamics}
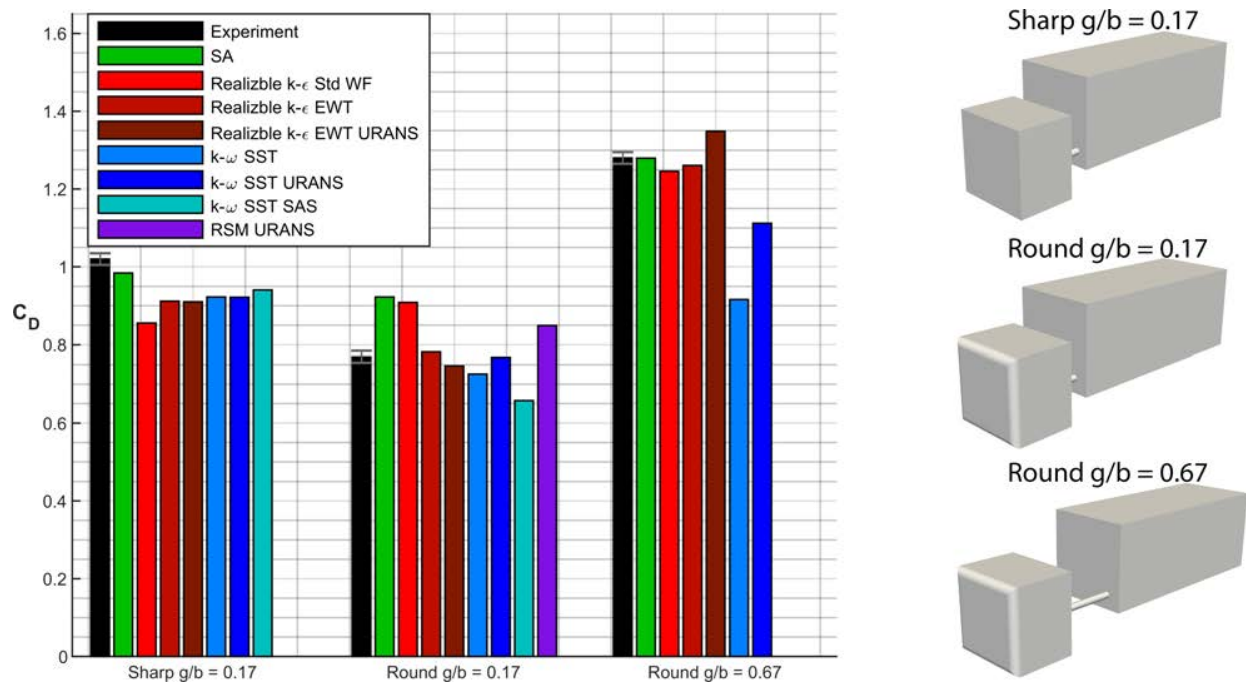

Figure 19: Comparison of the predicted drag coefficient of several RANS turbulence models to the wind tunnel measurements [23] for three geometrical configurations. Simulation data only exist for the $g / b=0.17$ gap distance for the $k-\omega$ SST SAS model, while only for the round $g / b=0.17$ body for the RSM model. A widespread of the predicted drag coefficient is seen among the Allan body configurations, as no model can predict the drag accurately for all configurations.

For the rounded front, more substantial discrepancies between the models are seen, particularly when considering both gap distances. However, the realizable $k-\varepsilon$ EWT model agrees well with the measurements for both gap distances with the rounded front. For the smaller gap distance $(g / b=0.17)$, the effect of the near-wall modeling is seen to make a significant difference for the realizable $k-\varepsilon$ model, as the Std WF results in much higher drag. This is explained by the pressure distribution on the front face of the rear box (denoted rb_ff), Figure 20. The rb_ff surface is responsible for the majority of the full body's drag force; thereby, the size of the stagnation region strongly affects the overall drag estimation for the models.

Two distinct types of stagnation pressure regions are seen for the models, where the SA, realizable $k-\varepsilon$ Std WF and RSM models result in a large stagnation zone on the top part of the surface, being the reason for the overpredicted drag. Significantly smaller stagnation region, and thereby lower drag, is seen for the $k-\omega$ SST based models, where the stagnation region is focused more at the top corners on rb_ff. The size of the stagnation region for the realizable $k-\varepsilon$ EWT model is in between the size of the other models and is the main reason for the more accurately predicted drag. The pressure distribution on the rb_ff is strongly connected to the flow over the front of the box, where flow separation is seen for some models, Figure 21. No or small separation results in more high-energy flow impinging the rb_ff, and thereby a large stagnation region. A substantial separation results in a recirculation region, directing the high-energy flow against the top part of the rb_ff, thereby acting similarly to a roof air deflector and reducing the drag. The latter 

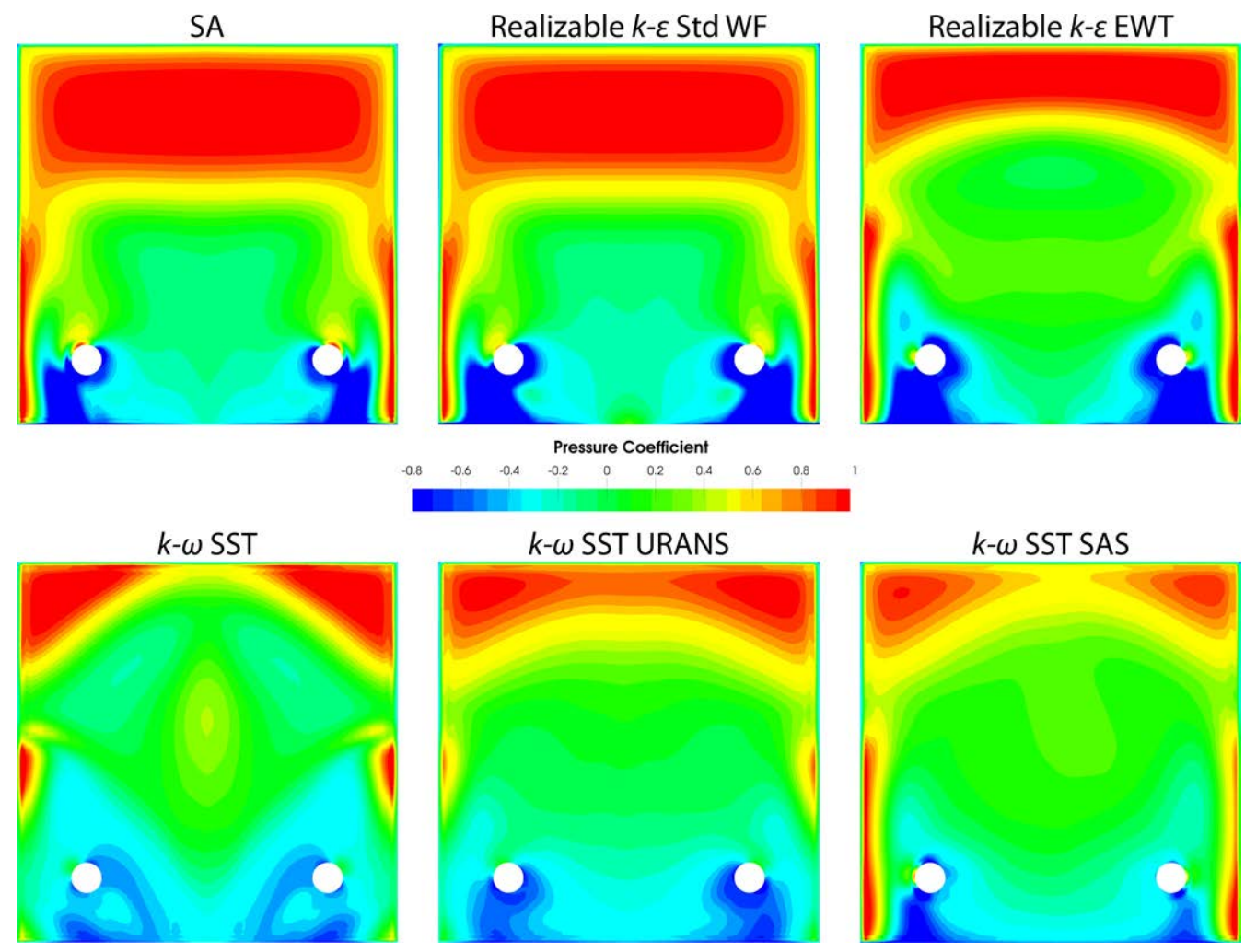

Figure 20: Time-averaged pressure coefficient for some of the investigated RANS models on the rear box front face ( $\left.r b \_f f\right)$ for the Allan body with rounded front and short gap distance $(g / b=0.17)$. A significant difference of the stagnation pressure for the top part of the surface is seen as an effect of differently captured separations around the front box.

behavior is seen for the $k-\omega$ SST based models, while no or small separation is seen for the SA and realizable $k-\varepsilon$ Std WF models.

Similar behavior is also seen for the large gap distance $(g / b=0.67)$, where the realizable $k-\varepsilon$ (including both wall functions) and SA models agree well with the measurements. Here as well, the drag is dependent on the pressure distribution on the rb_ff and hence the flow over the front curvature of the front box, Figure 22.

As for the smaller gap distance, it is seen that the SA and realizable $k-\varepsilon$ models predict a smaller separation than seen for the $k-\omega$ SST model. This is typical behavior for the realizable $k-\varepsilon$ and $k-\omega$ SST models and can be explained by investigation of how the models introduce turbulent viscosity in the near-wall region, Figure 23. Significantly higher turbulent viscosity in the near-wall region at the beginning of the front curvature is seen for the realizable $k-\varepsilon$ model compared to the $k-\omega$ SST model, resulting in more turbulence in the near-wall region and suppressed separation, which is a typical behavior of these models. 


\section{Important Factors for Accurate Scale-Resolving Simulations of Automotive Aerodynamics}
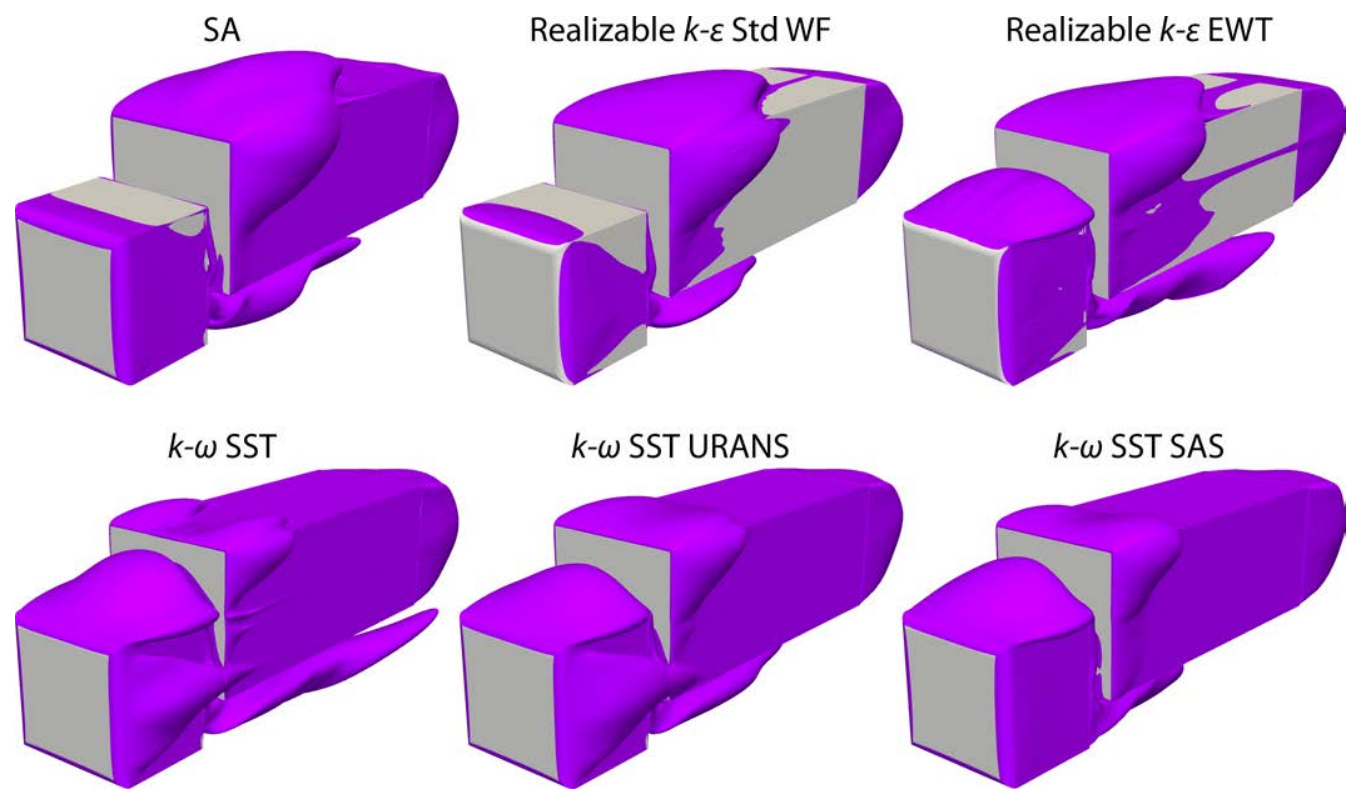

Figure 21: Iso-surface of the time-averaged zero total pressure for some of the investigated RANS models for the rounded $g / b=0.17$ Allan body configuration. Distinct differences of the pressure losses are seen around the front box of the Allan body. No or small separations are seen for the SA and realizable $k-\varepsilon$ Std WF models, while significantly larger separations are seen for the other models, especially the $k-\omega$ SST based models.

Running the RANS models in unsteady mode (URANS) does not make a significant difference in the model's main behaviors, except for the $k-\omega$ SST model at $g / b=0.67$ which slightly improved its behavior. The SAS model does, unfortunately, not bring any advantages either here and suffers from premature separation, just like the $k-\omega$ SST model it is based on. The SAS model's ability to switch to SRS mode when enough unsteadiness exists should theoretically be well suited for the Allan body, as unsteady separations and complex flow in the gap exists. To ensure sufficient temporal resolution of the unsteadiness, the used time-step size for the SAS simulations was fine enough to ensure values of $C F L_{\max } \leq 1$, being much finer than needed for the SAS model [45]. The URANS models ran for a larger time-step size $\left(C F L_{\text {max }} \approx 50\right)$, to capture the larger time-scales affecting the mean flow unsteadiness discussed in Section 2.3.2.

The Linear Pressure-Strain RSM model was also tested and showed reasonable accuracy for the rounded front and small gap distance. However, a typical problem with RSM models is their lack of robustness to reach convergence, especially for steady-state simulations. Therefore, transient mode with small time-steps was needed to reach sufficient convergence of the residuals and forces. This makes the RSM model expensive (for being a RANS model), and together with its lack of robustness, it is not suitable for industrial applications. 


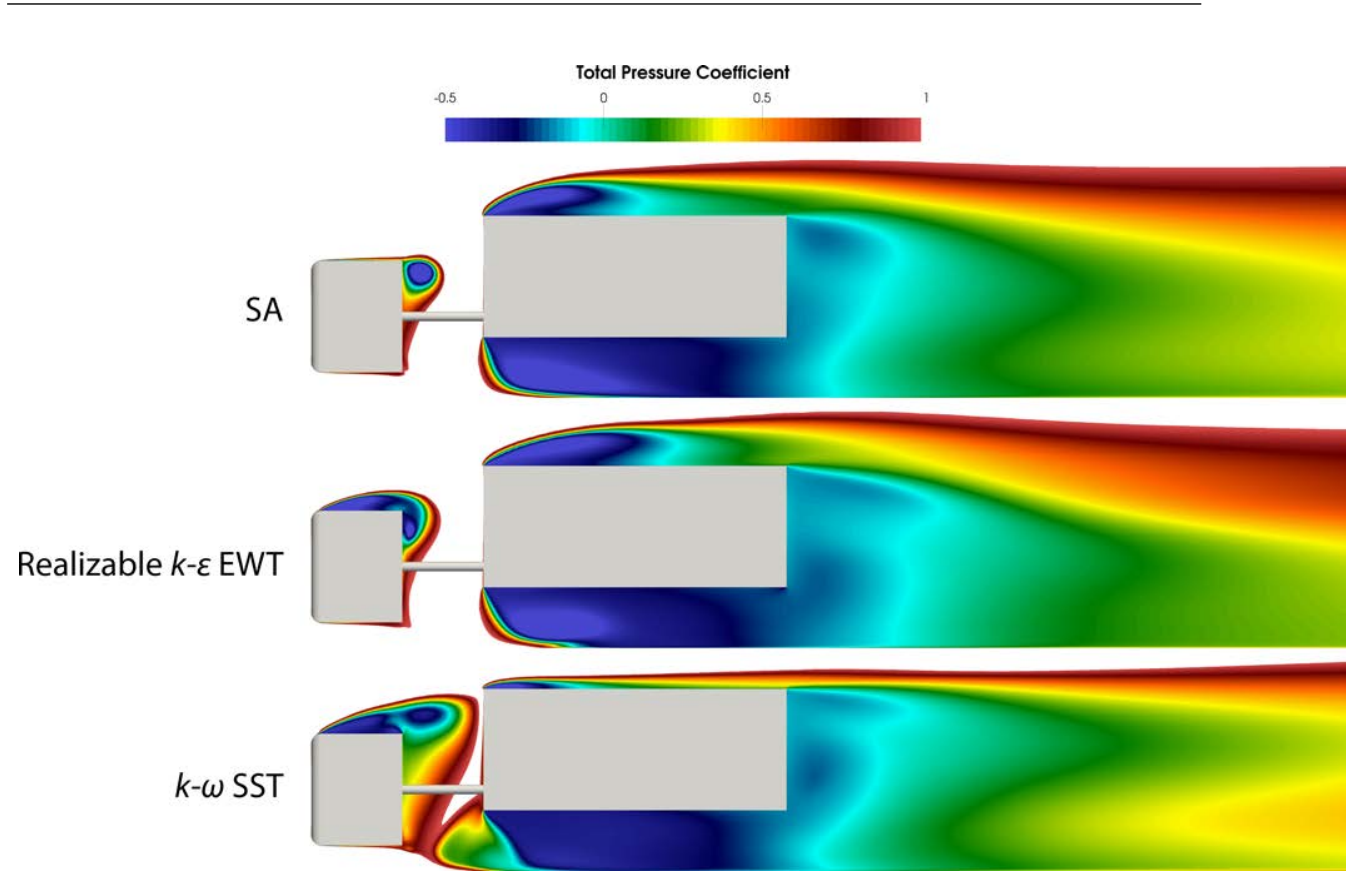

Figure 22: The time-averaged total pressure at the domain symmetry plane $(y=0)$ for the SA, realizable $k-\varepsilon$ EWT and $k-\omega$ SST models for the rounded $g / b=0.67$ Allan body configuration. The turbulence models predict different sizes of the flow separation around the front box. Note how the largest separation $(k-\omega$ SST model) acts as a roof air deflector and reduces the separation on the top part of the rear box.

From the RANS turbulence model investigation, the realizable $k-\varepsilon$ EWT model is in most agreement with the measurements and is therefore chosen for an extensive investigation of the gap distance for the rounded front, Figure 24 panel a. The realizable $k-\varepsilon$ EWT model captures the measured drag change well for the gap distance range of $g / b=0.17-0.45$. The distribution of drag for the surfaces in the gap rb_ff and the front box base face (denoted fb_bf) are also in good agreement with the measurements, Figure 24 panel b. Important to consider is that the measured drag is obtained by an area integration of the 49 pressure sensors (for each surface), which is a rather coarse estimation of the drag and therefore includes a significant error range in Figure 24 panel b.

Some interesting trends exist in the measurement data and is as well seen for the simulations, which is that drag mainly changes for rb_ff with increased gap distance, while the drag from fb_bf is rather constant with the gap distance. 


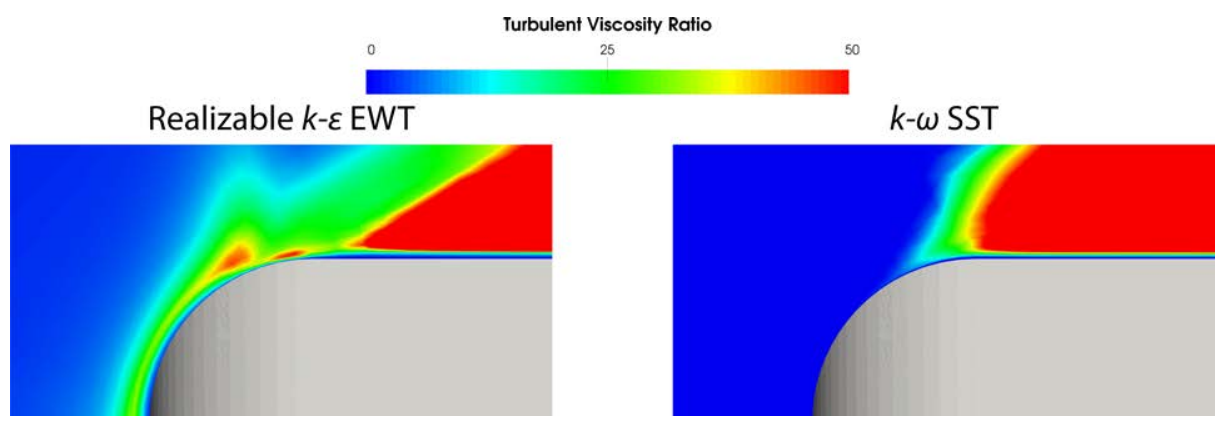

Figure 23: The turbulent viscosity ratio $\left(\nu_{t} / \nu\right)$ for the realizable $k-\varepsilon$ EWT and $k-\omega$ SST models for the rounded $g / b=0.67$ Allan body configuration. The realizable $k-\varepsilon$ EWT model generates significantly higher turbulent viscosity than the $k-\omega$ SST model, resulting in more introduced turbulent kinetic energy and thereby more attached flow.

In Paper I, the realizable $k-\varepsilon$ EWT model is used to investigate the effect of adding curvature to the top edge of rb_ff. From a parameter study of the curvature radii and gap distance, it is seen that a significant drag decrease is achieved for a radius of at least $100 \mathrm{~mm}$ for a full-scale truck. An $80 \mathrm{~mm}$ radius was then implemented on a full-scale truck fitted with a swap body, as a larger radius would require significant modification of the swap body walls. Over a testing period, a $3 \%$ fuel consumption reduction was achieved compared to the previous sharp-edged swap body. Showing that even small, but fundamental, geometrical changes can result in significant fuel savings.

(a)

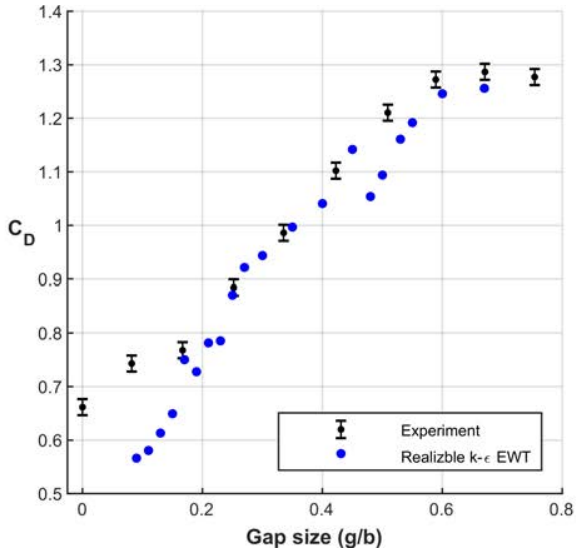

(b)

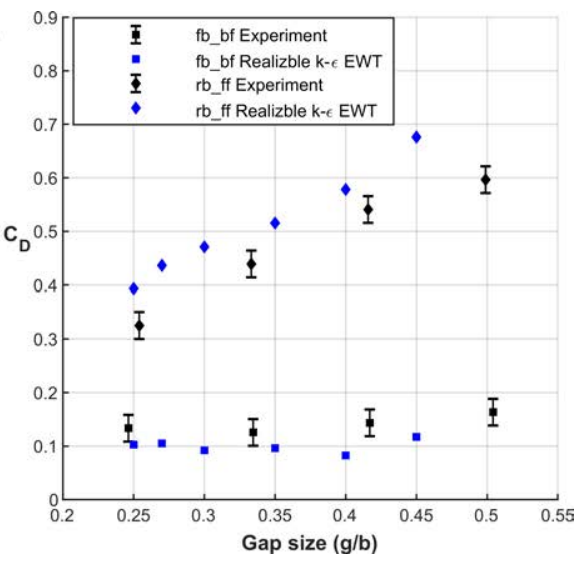

Figure 24: Drag coefficient against the gap distance for the rounded Allan body (a). The realizable $k-\varepsilon$ EWT model captures similar behavior as seen in the measurements [23], except for $g / b<0.17$ and $0.45>g / b<0.6$. In (b), good agreement between the realizable $k-\varepsilon$ EWT model and the pressure drag measurements are seen for the front box base face (fb_bf) and the rear box front face ( $\left.r b \_f f\right)$. Note how mainly the drag for the rb_ff is affected by the gap distance. 


\subsection{Resolving Turbulence - Less is More}

By modeling all the turbulent behavior (RANS) it is, as previously seen, difficult to achieve high accuracy for different vehicle geometries, but can work well for certain cases and conditions. However, for aerodynamic development of vehicles, high certainty of achieving accurate results is needed, especially since the introduction of WLTP and similar regulations for passenger cars and other vehicles, respectively. The investigated RANS models (except RSM) apply the isotropic eddy viscosity hypothesis proposed by Boussinesq [29]. As, especially, the larger eddies are not necessarily isotropic, thereby hard to model accurately, this assumption can introduce significant errors.

Even though SAS (and URANS to some degree) can resolve some of the larger turbulent structures, if scale separation exists, it is seemingly not enough to achieve high accuracy for the flow field around the Allan body. Using LES that resolves most of the turbulence (Figure 8) is, therefore of interest, to see how accurate, current available turbulence modeling methods are and where the current limits of commercial CFD software are. With more resolving of the turbulent structures, a finer mesh and temporal resolution are needed, greatly increasing the computational cost. Some modeling of the turbulence is needed, as the computational cost for DNS is far beyond the scope of this thesis and will likely not be available for industrial aerodynamic simulations before 2080 [83]. Wall resolving LES is also far from realistic within the industry for automotive aerodynamics. However, it is within reach, of reasonable computational costs, for generic bodies at low to moderate Reynolds numbers.

In Paper I, a comparison to LES simulations [53] is included. The LES is seen to be accurate for the smaller gap distance but far off the measurement for the large gap distance $(g / b=0.67)$. The used mesh size in [53] is in the same order as the one used for the RANS simulations, most likely being too coarse for wall resolving LES. To assess the needed eventual necessary mesh resolution, tests were performed with LES for mesh sizes up to 100 million cells. However, the near-wall mesh resolution (explained in Section 2.5) could still not be met, indicating a need for even finer mesh resolution, particularly in the near-wall region, and high computational costs. The Allan body also has a relatively low flow feature interaction for the lower gap distances, as the flow around the front curvature strongly determines the overall flow behavior, and the experimental measurements are rather coarse compared to more recent aerodynamic measurements and is therefore not suitable to test models where high accuracy is expected.

In 2015, Venning et al. [67] published high-resolution PIV measurements of the wake flow for the Ahmed body at a Reynolds number of $9.34 \cdot 10^{4}$, based on the length of the body. The low Reynolds number makes it possible to investigate the accuracy of current LES methods with sufficient mesh resolution for a reasonable computational cost. The low Reynolds number does, however, introduce a more substantial laminar part of the flow and thereby make the transition to turbulence important as well. The effect of laminar flow might also be of more importance in future car development, as cars aerodynamic design consistently becomes more efficient, and reducing the turbulence (with its pressure losses) is a step towards it. The Reynolds number can have a significant effect on the flow around vehicles and bluff bodies. However, the flow behavior over the rear part of the 


\section{Important Factors for Accurate Scale-Resolving Simulations of Automotive Aerodynamics}

Ahmed body is known to have low sensitivity to the Reynolds number and is, therefore, possible to investigate at low Reynolds numbers $[67,68,84,85]$.

To control the amount of diffusion from the smaller turbulent scales, scale-resolving models usually involve SGS models. The SGS model models the behavior and effects of these scales on the overall flow field and is therefore supposed to have a rather small effect on the overall flow field behavior. However, studies of flow around bluff bodies have shown it can cause significant differences [86, 87].

In Paper II, the effects of different SGS modeling approaches are investigated on the Ahmed body. The Ahmed body is a simple generic car body, which is one of the most investigated car bodies due to its complicated flow over the rear of the body. Three commonly used SGS
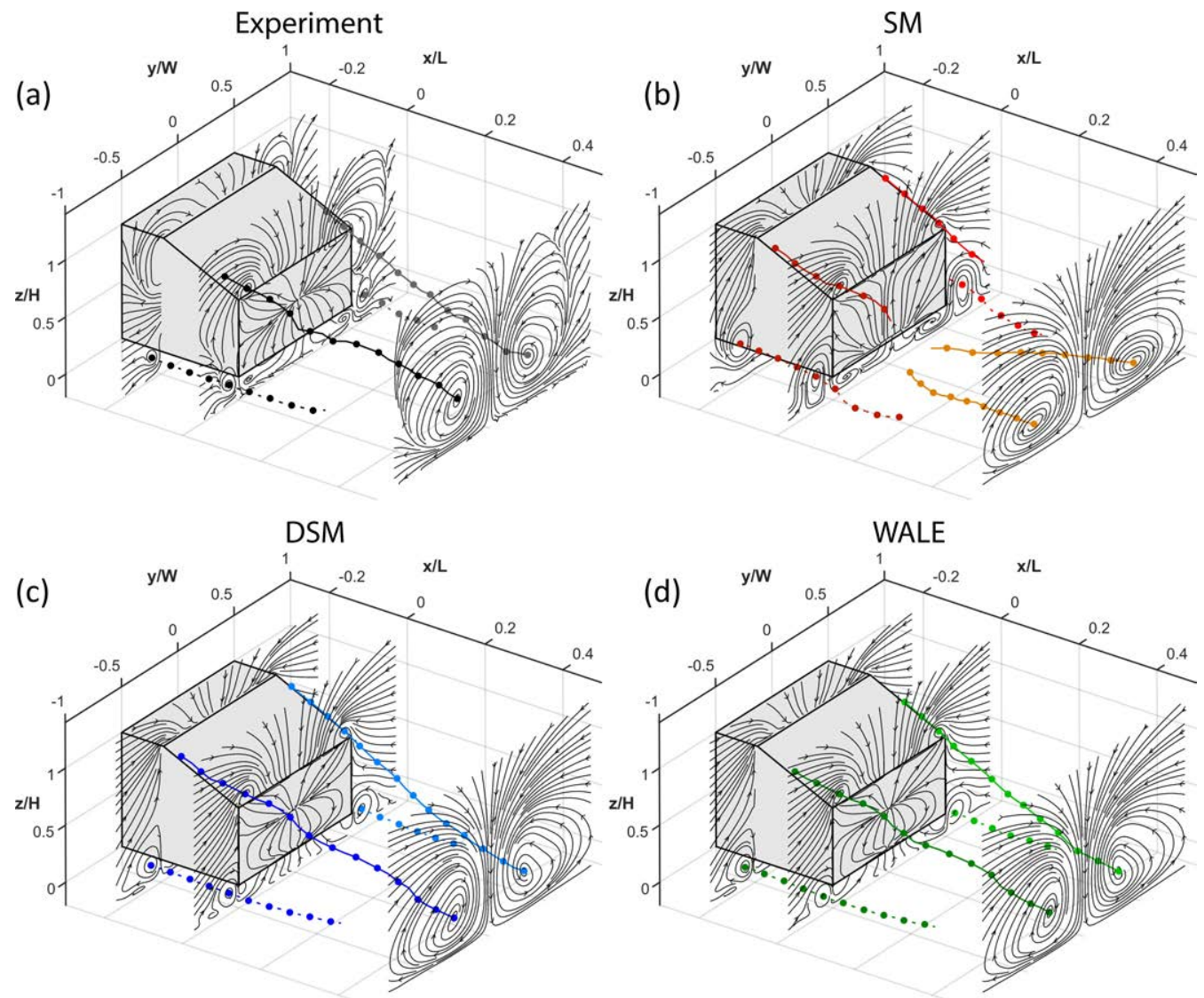

Figure 25: Time-averaged 2D streamlines in three yz-planes for the PIV measurement (a), SM (b), DSM (c), and WALE (d) for the rear of the Ahmed body. Solid lines represent the C-pillar vortex center, and the dotted lines represent the corner vortices. The orange dotted lines in panel b, represent the vortex center for the far wake of the SM SGS model. Reprinted from Paper II with permission from ASME. 
models (SM, DSM and WALE, see section 2.3.2) are compared against high-resolution PIV measurements of the flow over the rear part of the body. In Figure 25, the time-averaged vortex center from the C-pillar and corner vortices are seen together with 2D streamlines in three planes for the PIV measurement, and the three investigated SGS models. All the evaluated SGS models predict the behavior of the C-pillar vortices similarly to the PIV measurement until the end of the body. Here, the SM SGS models C-pillar vortices break down due to vastly different behavior of the rear wake. Strong similarities between the results from the DSM and WALE SGS models are seen, correlating well to the PIV measurement for the whole wake, more discussed in Paper II. Notably, the SM SGS model predicts similar flow behavior for the downstream plane $(x / L=0.5)$, but the origin of the large counter-rotating vortices origin from the underside of the lower part of the wake at $x / L=0.05$ (orange lines in Figure 25 panel b). Significantly larger corner vortices are also seen for the SM SGS model, while the ones for DSM and WALE SGS models are of similar size and trajectory, as seen in the experimental measurement.

The reason for the different wake behavior of the SM SGS model is caused by a too large separation of the front airfoil stilt, Figure 26 panel a. Unfortunately, no experimental data exist for the underbody flow, and hence full certainty of the underbody flow cannot be obtained. However, as both the DSM and WALE SGS models accurately predict the wake and its behavior, and, especially, the path of the corner vortices, there is high certainty these two models are capturing the underbody flow with high accuracy. The SM SGS model also overpredicts the separation at the front of the Ahmed body, compared to the DSM and WALE SGS model, Figure 26 panel c.

Due to the low Reynolds number $\left(R e_{L}=3.34 \cdot 10^{4}\right)$, a significant part of the flow upstream and around the front of the Ahmed body is laminar, Figure 27. The cause of the larger separations for the SM SGS model at the front stilt and front is explained by the lower instabilities inside the boundary layer than seen for the other two SGS models. The SM SGS model instead introduces more turbulent viscosity than the DSM and WALE SGS models, thereby dampening the instabilities. Comparing the turbulent kinetic energy (including both the modeled and resolved parts) inside the boundary layer at these regions reveals significant lower turbulent kinetic energy for the SM SGS model, compared to the DSM SGS model, even though its higher turbulent viscosity, Figure 27 panel b. This results in less momentum in the boundary layer and an earlier and thereby larger separation. Even though the Ahmed body consists of a long mid-body, some turbulent kinetic energy still exists at the beginning of the slant, Figure 27 panel a. As an effect of the larger separation at the front for the SM SGS model, more turbulence is present at the beginning of the slant, resulting in slightly smaller separation over the slant, than seen for the DSM and WALE SGS model, Figure 26. The DSM and WALE SGS models predict the flow over the slant very similarly, with only small differences in the skin friction field.

Both the DSM and WALE SGS models are formulated to achieve correct near-wall behavior, with the turbulent viscosity automatically going to zero in laminar flow, thereby at the wall, and does not need a damping function. The SM SGS model needs a damping function ( $\kappa d$ in Equation 34$)$ to achieve this and is seemingly not as accurate. 
High accuracy is possible to achieve with LES models existing in today's commercial CFD software. However, it is essential to consider the SGS modeling, especially if no or low turbulence is present in the near-wall region at curvatures for important regions, which can exist for small parts subjected to the freestream (e.g., mirrors). The DSM SGS model is from this investigation, the preferred model, as the dynamic property makes it more suitable for a wider variety of flows, even though it increases the already high computational cost of LES.

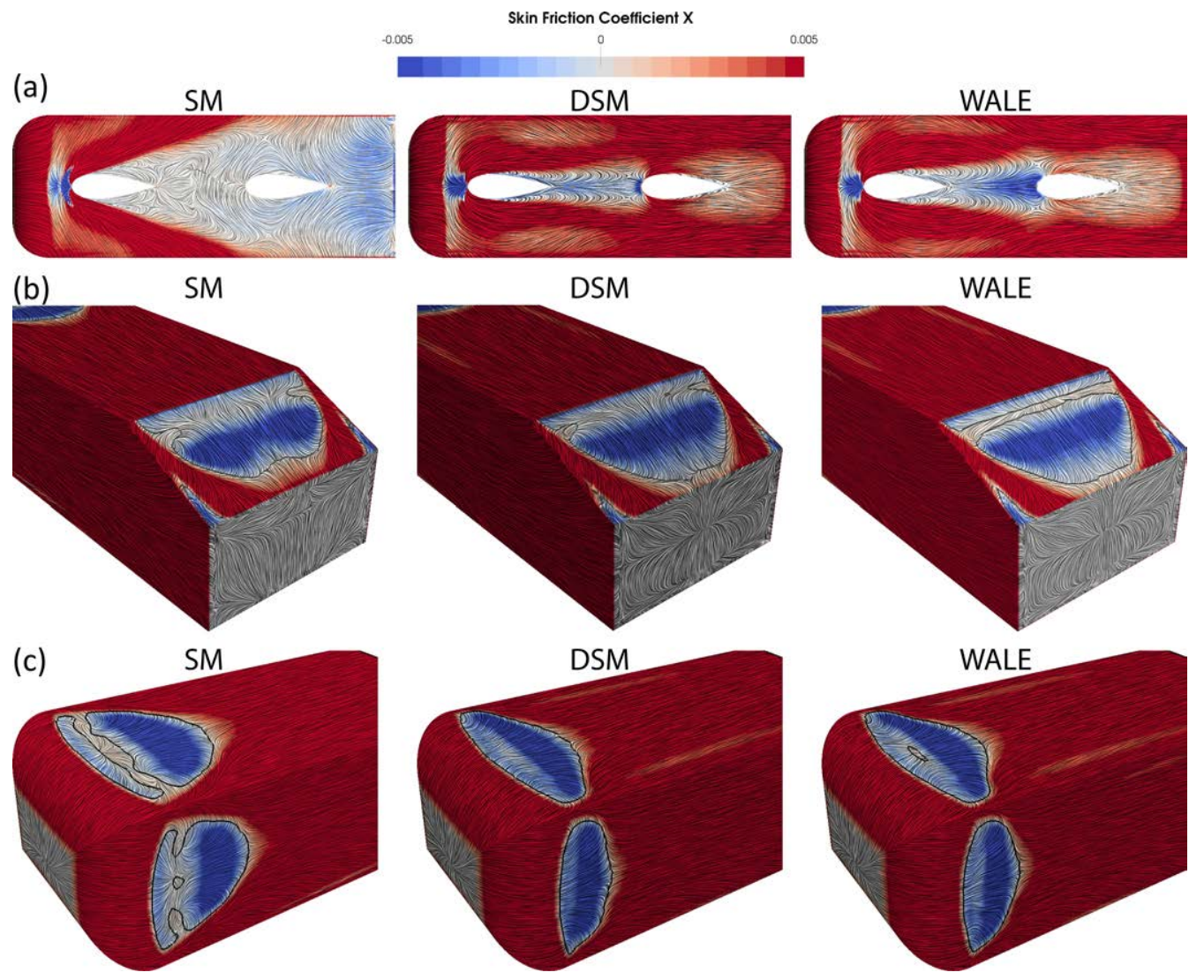

Figure 26: Skin frictions surface lines and the x-skin friction coefficient for the three investigated SGS models for the Ahmed body. (a) Significant differences are seen for the underbody flow field, where a large separation occurs for the SM SGS model. (b) Similar separation length (black lines are $\mathrm{x}$-skin friction $=0$ ) is seen at the slant for the DSM and WALE SGS models. More upstream turbulence results in a smaller separation at the slant for the SM SGS model. (c) The SM SGS model overpredicts the separation at the front, compared to the DSM and WALE SGS models. 


\section{Modeling Turbulence in Specific Regions}

(a) Normalized Resolved Turbulent Kinetic Energy
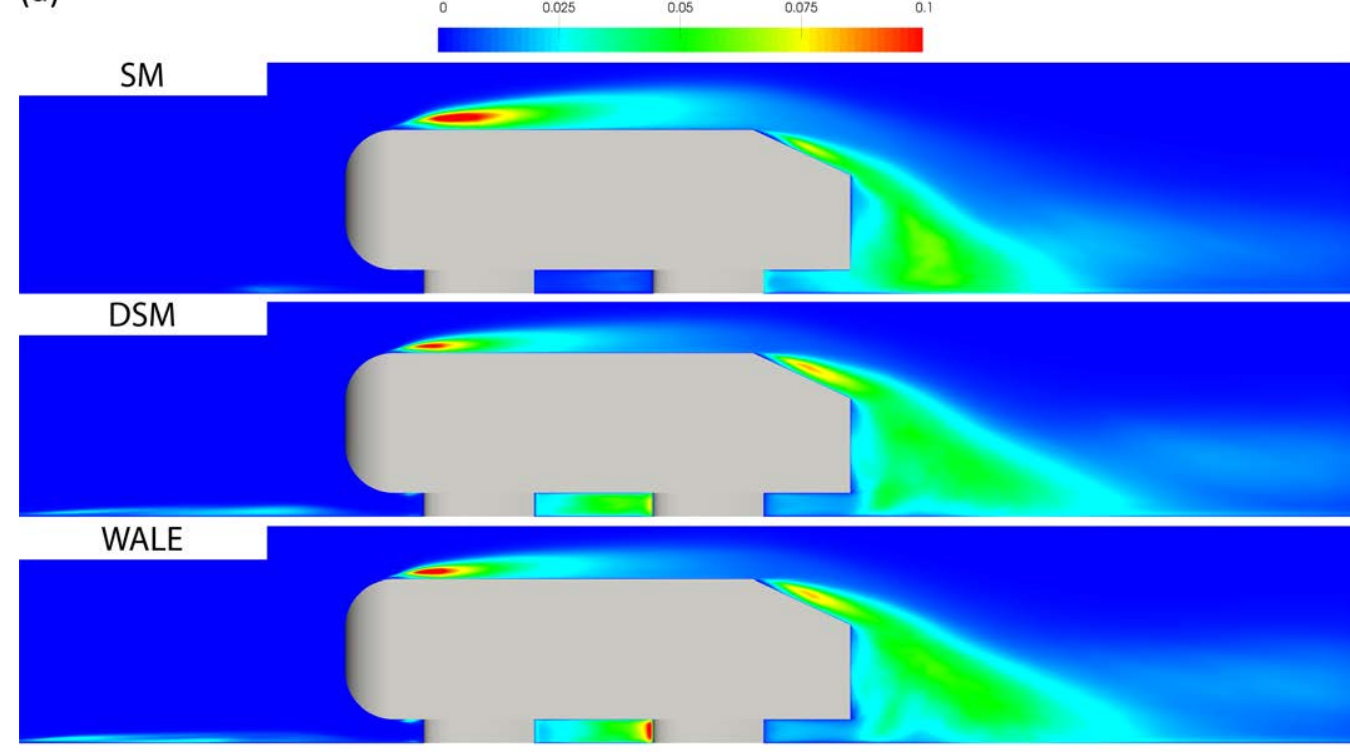

(b)

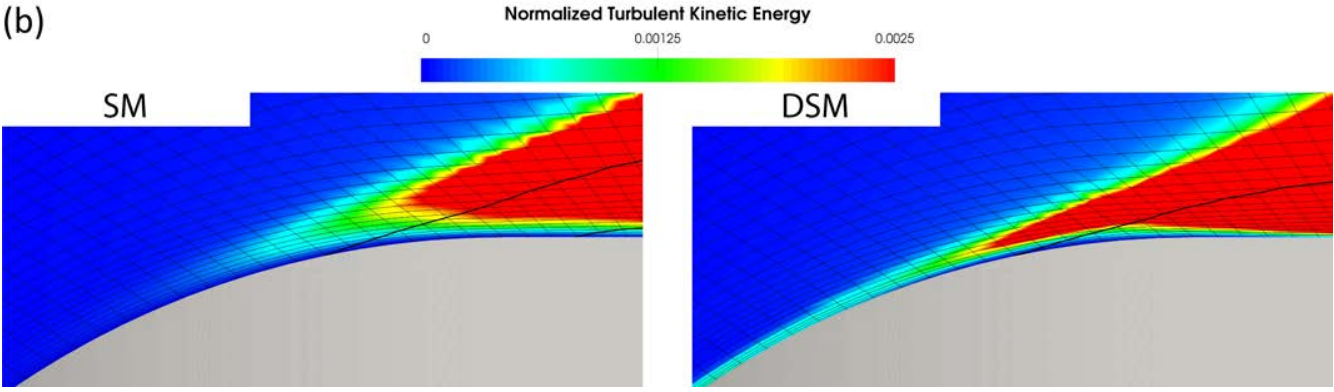

Figure 27: (a) The resolved turbulent kinetic energy for the three SGS models at the domain symmetry plane, $y=0$. Note the lack of resolved turbulent kinetic energy upstream of the body adjacent to the test-section floor for the SM SGS model. (b) Comparison of the turbulent kinetic energy (including both the resolved and modeled part) at the round front of the Ahmed body. Similar to upstream the body, no turbulent kinetic energy exists upstream and results in a larger separation. Thick black line is the time-averaged $x$-velocity equal to zero.

\subsection{Modeling Turbulence in Specific Regions}

The need for a fine mesh and temporal resolution, especially in the near-wall region, for LES makes it very computationally costly for the high Reynolds numbers vehicles operate in. A Simulation using LES was performed for the DrivAer car model at $R e_{L}=1.56 \cdot 10^{6}$, eight times lower than a full-scale car at $140 \mathrm{~km} / \mathrm{h}$. The mesh consisted of 458 million cells, with more than $80 \%$ of the cells in the near-wall region, and resulted in a computational cost of 2.8 million core-hours for simulation of 20 convective flow units on the Tetralith 


\section{Important Factors for Accurate Scale-Resolving Simulations of Automotive Aerodynamics}

supercomputer [88]. This amount of computational cost for a single simulation is now and for the foreseeable future unfeasible in the automotive industry, and therefore other computationally cheaper methods are necessary. As most cells are needed in the nearwall region for resolving the small turbulent structures, modeling this region with RANS makes it possible to reduce the mesh resolution, hence reducing the computational cost significantly. There exist several hybrid RANS-LES methods, where one of the most commonly used is DES [59], explained in Section 2.3.2.

There exist several variations of the originally proposed DES method, as different RANS models and/or shielding techniques of the RANS layer have been proposed. To better understand which DES models and methods might be suitable for automotive aerodynamic simulation, several variations are tested on the Ahmed body. The same mesh and numerical set-up as for the LES SGS modeling investigation is used, where the mesh might be unnecessary fine for hybrid RANS-LES models but thereby also test the RANS shielding properties of the models. Sufficient shielding of the RANS region for fine mesh resolution is critical to ensure that the model do not prematurely switches to LES for the near-wall region, thereby introducing under-resolved turbulent structures and stresses, which can reduce the accuracy significantly.
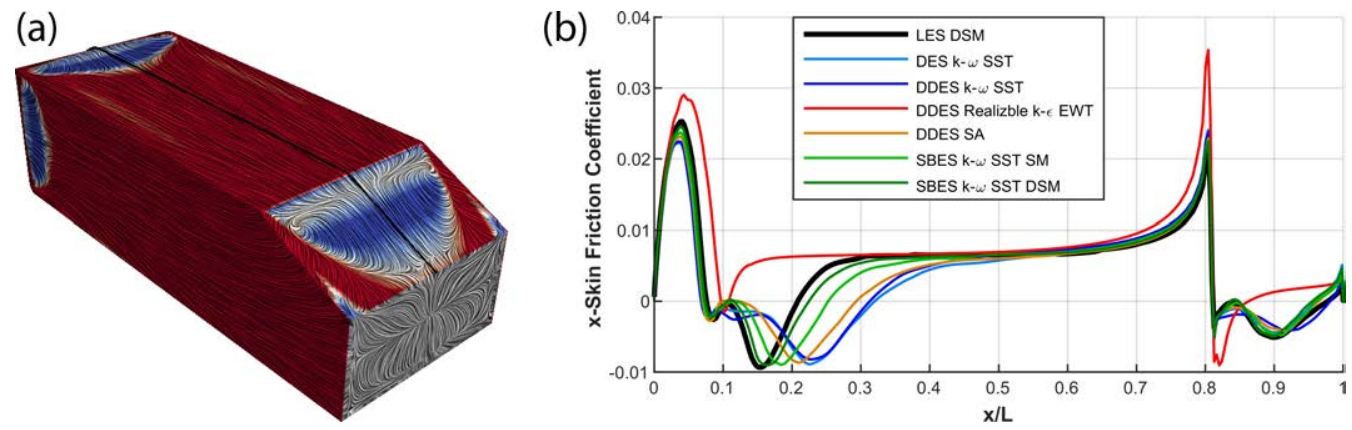

Figure 28: (a) Streamwise $(x)$ skin friction for the LES DSM SGS model for the Ahmed body. (b) Several DES methods and variations are evaluated for the x-skin friction coefficient for the black line seen in (a) and compared to the LES DSM SGS model. Significant differences for the separation at the front and over the rear is seen between the models.

In Figure 28 panel a, the streamwise (x) skin friction coefficient is seen for the Ahmed body simulated with LES. For the black line at the body symmetry line, the streamwise skin friction is evaluated for several DES methods and variations, Figure 28 panel b. Results from the LES with the DSM SGS model acts as a reference in this comparison, as it shows high accuracy in Paper II. Overall similar separation points are seen at the front of the body for all the models, except for the DDES realizable $k-\varepsilon$ EWT model, which barely predict any separation at all. The separation length differs significantly between the models, where the largest separation occurs for the DES and DDES with the $k-\omega$ SST model. The SBES models are, however, behaving very similar to the behavior of LES, especially when using the DSM SGS model in the LES region. Some differences are seen between using the SM or the DSM SGS models together with the SBES model. This indicates the importance of using a more advanced SGS model even for DES methods 
when laminar to turbulent transition is of importance.

As the LES model in DES and DDES models is analogous to the SM SGS model, an indication of sufficient and insufficient shielding can be seen between the SBES $k-\omega$ SST SM and the DES and DDES $k-\omega$ SST models. The much stronger shielding for the SBES model does even for this fine mesh ensure sufficient shielding of the RANS region. The small difference between the DES and DDES shows that even the "delayed" addition for the DDES model, is not enough to ensure RANS shielding for this type of fine meshes. As the SBES includes blending of the RANS model to the LES model, a faster transition from the RANS to LES region is possible, resulting in a smaller gray-area and thereby faster generation of resolved turbulence. The effect of this is seen in Figure 29, where the SBES $k-\omega$ SST DSM model achieve similar values of resolved turbulent kinetic energy as the LES DSM model for the upper part of the Ahmed body. The larger separation for DDES $k-\omega$ SST model is the reason for the model's higher resolved turbulent kinetic energy, which behavior is similar to the behavior of the LES SM model in Paper II. This is to be expected, as the SGS model in DES and DDES is analogous to the SM SGS model.

(a)

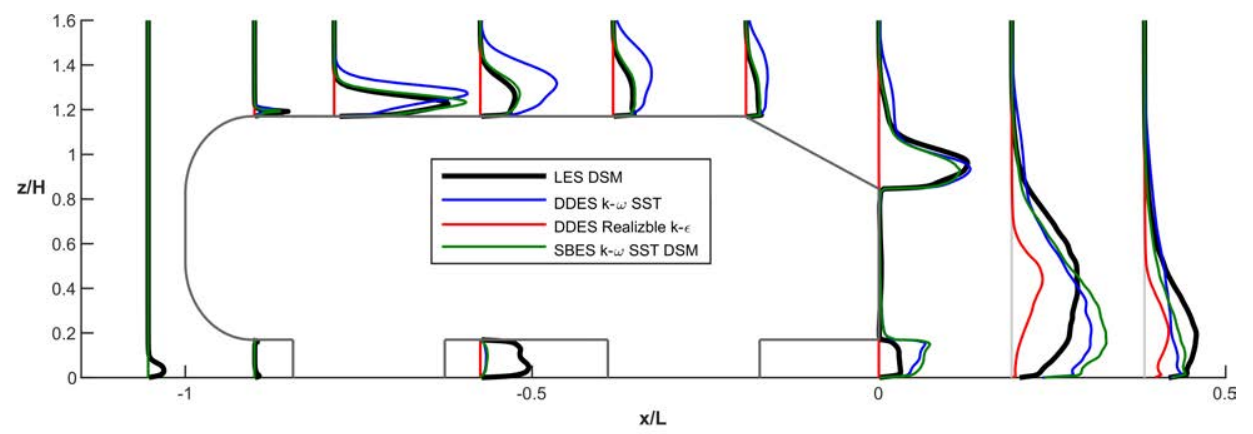

(b)
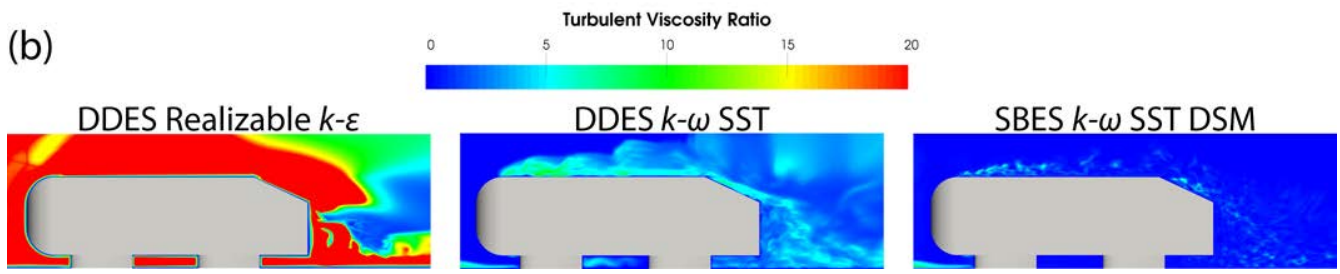

Figure 29: (a) Lines of resolved turbulent kinetic energy for the LES DSM SGS model and three hybrid RANS-LES models. Note that the DDES realizable $k-\varepsilon$ EWT model is not able to resolve any turbulence until downstream of the body. The SBES $k-\omega$ SST DSM model, is in good agreement with the LES DSM model, as an effect of faster transition between the RANS and LES regions. The different resolved turbulent kinetic energy for DDES $k-\omega$ SST and SBES $k-\omega$ SST DSM models at the underbody, and the lower part of the wake is in good agreement with the LES SM SGS model, seen in Paper II, as an effect of overpredicted separation at the front stilt. (b) Significant differences of the instantaneous turbulent viscosity ratio is seen for three hybrid RANS-LES methods. The lower turbulent viscosity ratio seen for the SBES $k-\omega$ SST DSM model, results in more rapid development of resolved turbulent structures. 


\section{Important Factors for Accurate Scale-Resolving Simulations of Automotive Aerodynamics}

Some differences of the resolved turbulent kinetic energy is seen for the wake, caused by overpredicted separation around the front stilt for the investigated hybrid RANS-LES models, except for the DDES realizable $k-\varepsilon$ EWT model which, as well here, predict attached flow around the stilts. Much higher values of turbulent viscosity is seen for the DDES realizable $k-\varepsilon$ EWT model, which suppresses the resolving of turbulent structures (hence resolved turbulent kinetic energy, Figure 29 panel a), and only some resolved turbulence is seen for the downstream part of the wake, Figure 29 panel b. Significantly lower turbulent viscosity is seen for the other DES and DDES models, as the SA and $k-\omega$ SST models introduce less turbulent viscosity in the near-wall region. Even lower turbulent viscosity is seen for the SBES model, which is an effect of the stress blending and faster transition to the LES region.

To evaluate if hybrid RANS-LES models also results in higher accuracy for other geometries, the SBES $k-\omega$ SST SM and DDES $k-\omega$ SST models are tested on the Allan body with a rounded front and gap distance of $g / b=0.50$. For this gap distance, the measured pressure distribution of the fb_bf and rb_ff exist, making it possible to determine more in detail where the models are accurate and not. For this gap distance, the realizable $k-\varepsilon$ EWT model is also seen to not perform as well of predicting drag, Figure 24, making it possible to see where this inaccuracy originates. This gap distance also cause more flow interaction for the Allan body, as not only the separation of the front determines the prediction of drag.

(a)

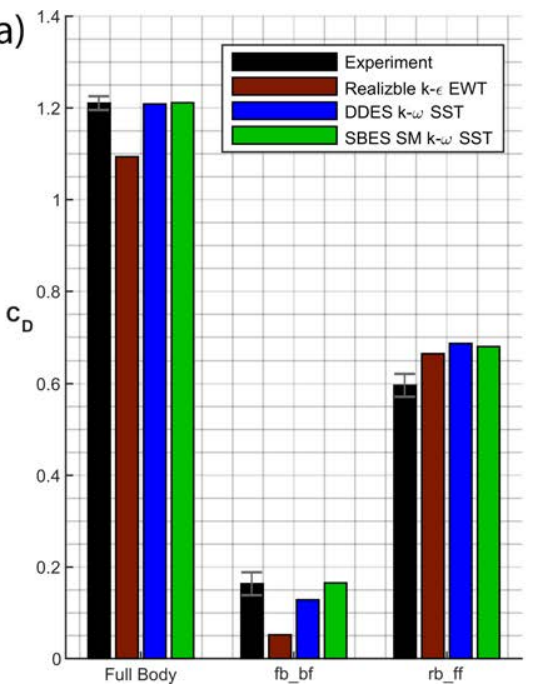

(b)

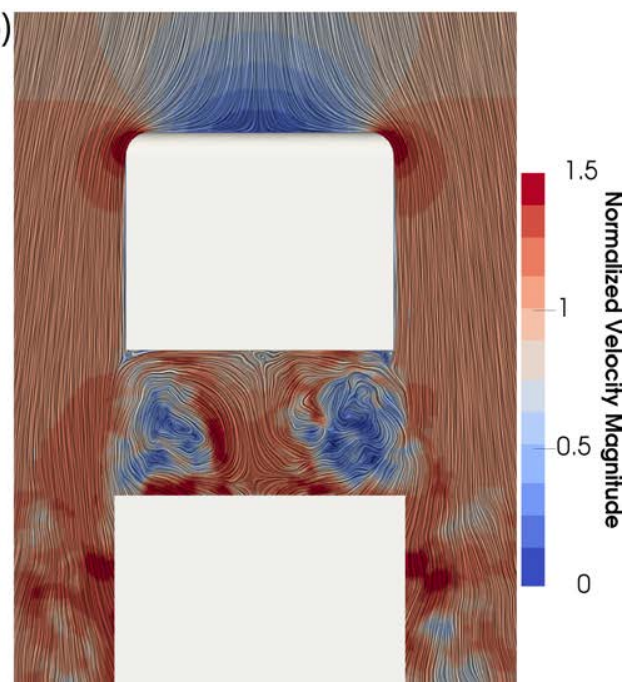

Figure 30: (a) Full body drag and for the fb_bf and rb_ff surfaces for the realizable $k-\varepsilon$ EWT, DDES $k-\omega$ SST and SBES $k-\omega$ SST SM models, compared to wind tunnel measurement [23]. A significantly better agreement with the measurements is seen for the hybrid RANS-LES models, especially for the fb_bf. (b) The better agreement is due to the realizable $k-\varepsilon$ EWT models' inability to accurately model the complex flow in the gap of the boxes.

In Figure 30 panel a, the drag from the full body and the fb_bf and rb_ff surfaces are compared to the measurements from [23]. Better correlation to the measurements is seen 
for the hybrid RANS-LES models, compared to the realizable $k-\varepsilon$ EWT model. This is especially true for the fb_bf surface, where the realizable $k-\varepsilon$ EWT model greatly under predicts the drag.
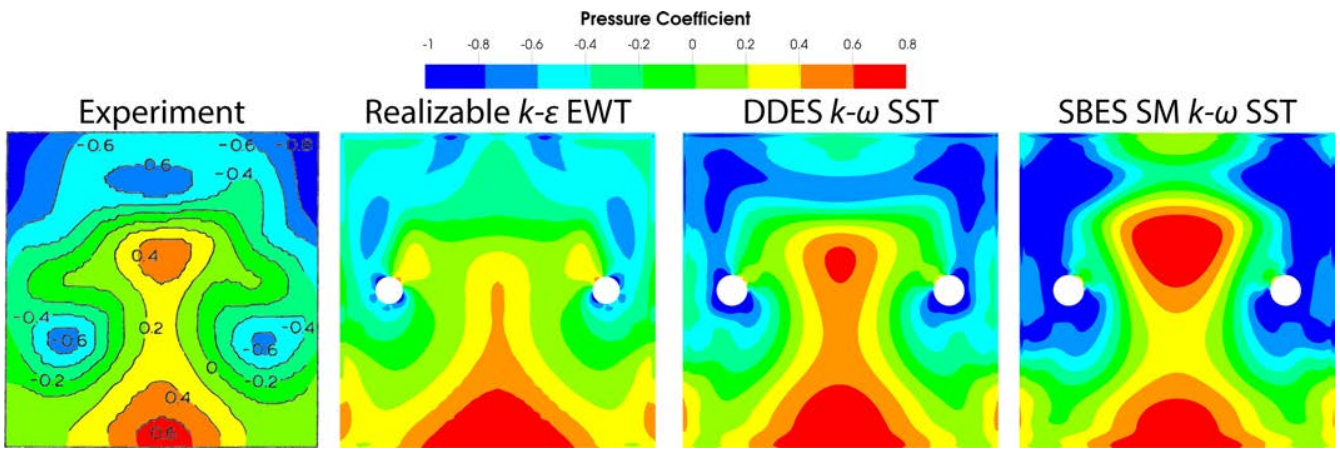

Figure 31: The pressure distribution of the fb_bf. Experimental data is from [23] and only consists of 49 pressure probes and does not account for the cylinders holding the boxes together. Better agreement with the measurement, especially for the top part, is seen for the DDES $k-\omega$ SST and SBES $k-\omega$ SST SM models.

The flow in the gap is very complex and consists of highly unsteady flow with turbulent scales of a variety of sizes, Figure 30 panel b. This is difficult for the realizable $k-\varepsilon$ EWT model to accurately predict and results in poor agreement to the measurement for the pressure distribution of fb_bf, Figure 31. Here, the hybrid RANS-LES models are more closely aligned with the measurement, in particular for the top part of the surface. For this surface, the DDES $k-\omega$ SST model is more closely matched to the measured pressure distribution, while the SBES $k-\omega$ SST SM model is closer to the measured drag. It is important to note that the pressure measurement is performed with only 49 pressure sensors, making it unreasonable to investigate the details in further depth.

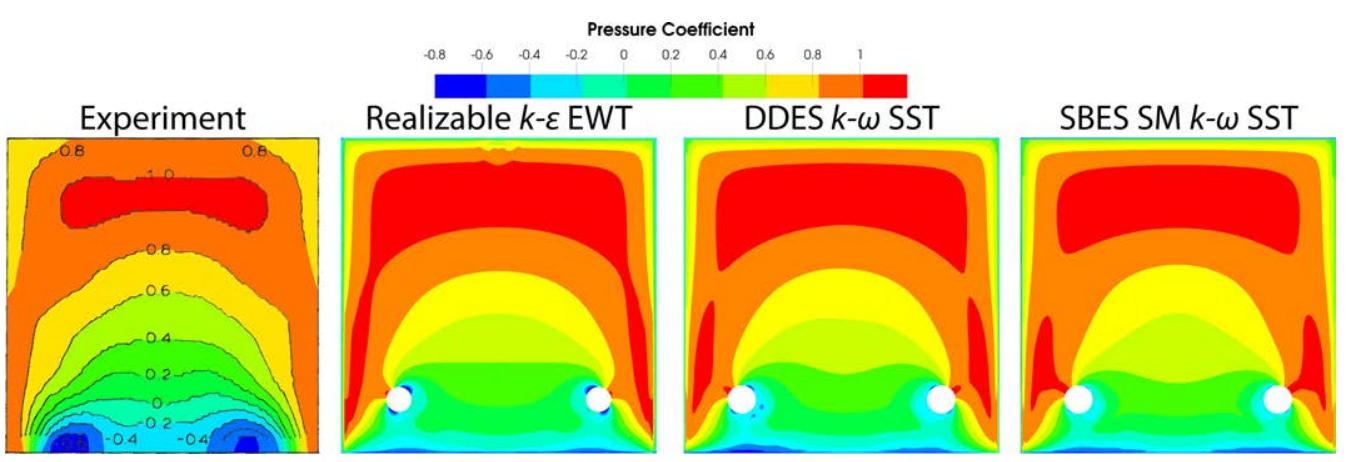

Figure 32: The pressure distribution of the rb_ff. Experimental data is from [23] and only consists of 49 pressure probes and does not account for the cylinders supporting the boxes. Similar behavior, and good agreement, is seen for all models, as the pressure distribution on this surface mainly is determined by the flow field around the front box. 


\section{Important Factors for Accurate Scale-Resolving Simulations of Automotive Aerodynamics}

For the rb_ff, all the models (including the realizable $k-\varepsilon$ EWT) are more closely matched to the measurement for both drag and pressure distribution, Figure 32. However, as previously discussed, this surface is much dependent on the separation on the curvature of the front box. A significant difference for the $k-\omega$ SST model is that it does not overpredict this separation when used together in a hybrid RANS-LES model, as large stagnation zones are seen on rb_ff for both the DDES $k-\omega$ SST and SBES $k-\omega$ SST SM models. Only a small separation on the top part of the front box is seen for these models and aligning with what is reported in [23].

\subsection{A Reality Check: Drag Reduction}

Light trucks are responsible for a large part of the greenhouse gas emissions from road transport [1, 89], Figure 1. Like lorries and tractor-trailers, a complete light truck can include parts from several manufactures, which influence the exterior shape and often results in less efficient aerodynamics. This is addressed in Paper III, where the aerodynamics of a light truck fitted with a swap body is investigated and improved.

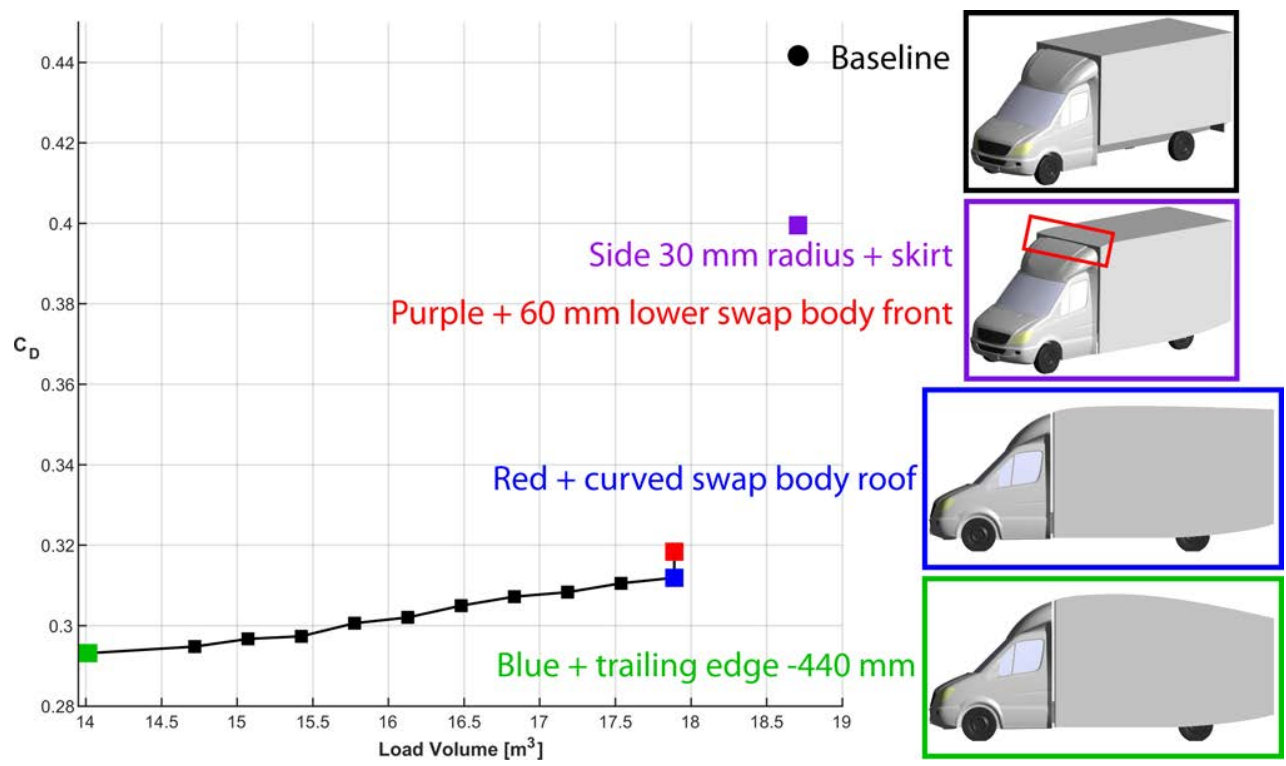

Figure 33: The drag coefficient and the usable loading volume of the swap body for the evaluated light truck concepts. Significant drag reduction with small losses to the volume loading is seen for small geometrical changes to mainly the top front part of the swap body. Streamlining the swap body results in even more reduced drag but to significant losses of the usable loading volume. 


\section{A Reality Check: Drag Reduction}

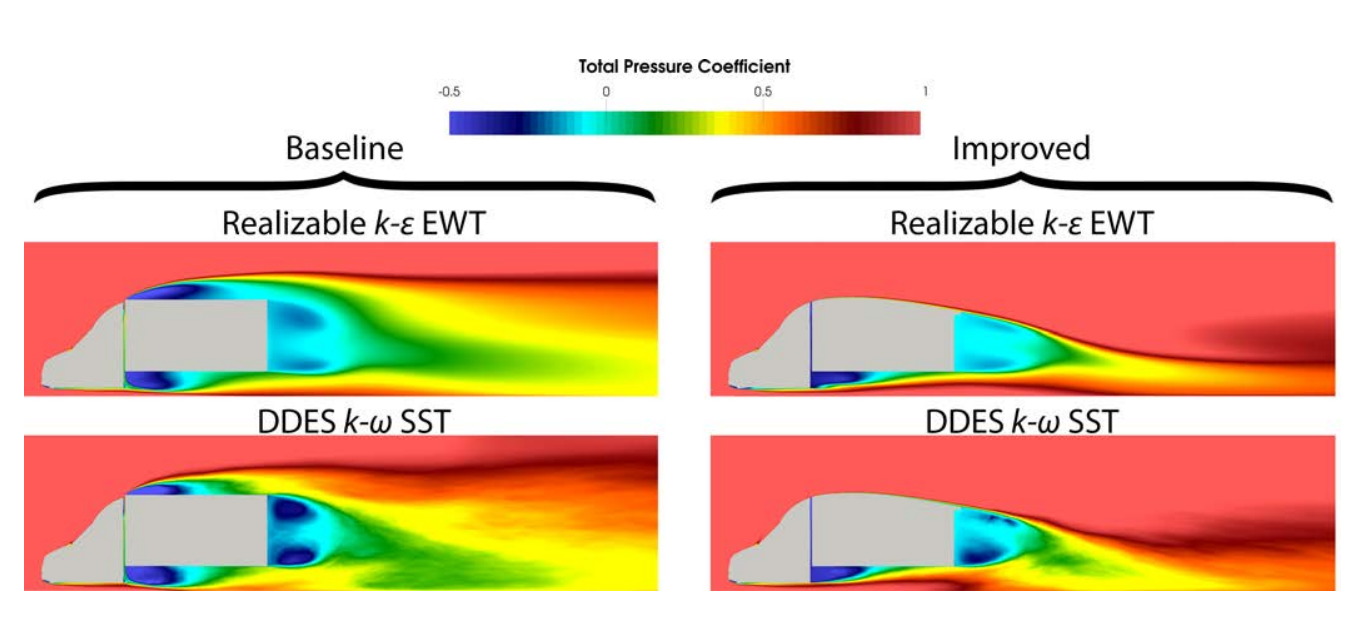

Figure 34: Comparison of the time-average total pressure coefficient for the baseline and improved concept with loading volume of $14.7 \mathrm{~m}^{3}$ (blue square in Figure 33) simulated with the realizable $k-\varepsilon$ EWT and DDES $k-\omega$ SST models. Similar behavior is seen between the turbulence models, but with greatly different magnitudes, especially in the rear wake. Added modifications remove the significant pressure losses seen for the top front part of the swap body and in the rear wake.

The realizable $k-\varepsilon$ EWT model is used for evaluating different geometrical changes of the light truck, as this model can capture the essential trends when investigating the gap distance for the Allan body (Figure 24) at a low computational cost.

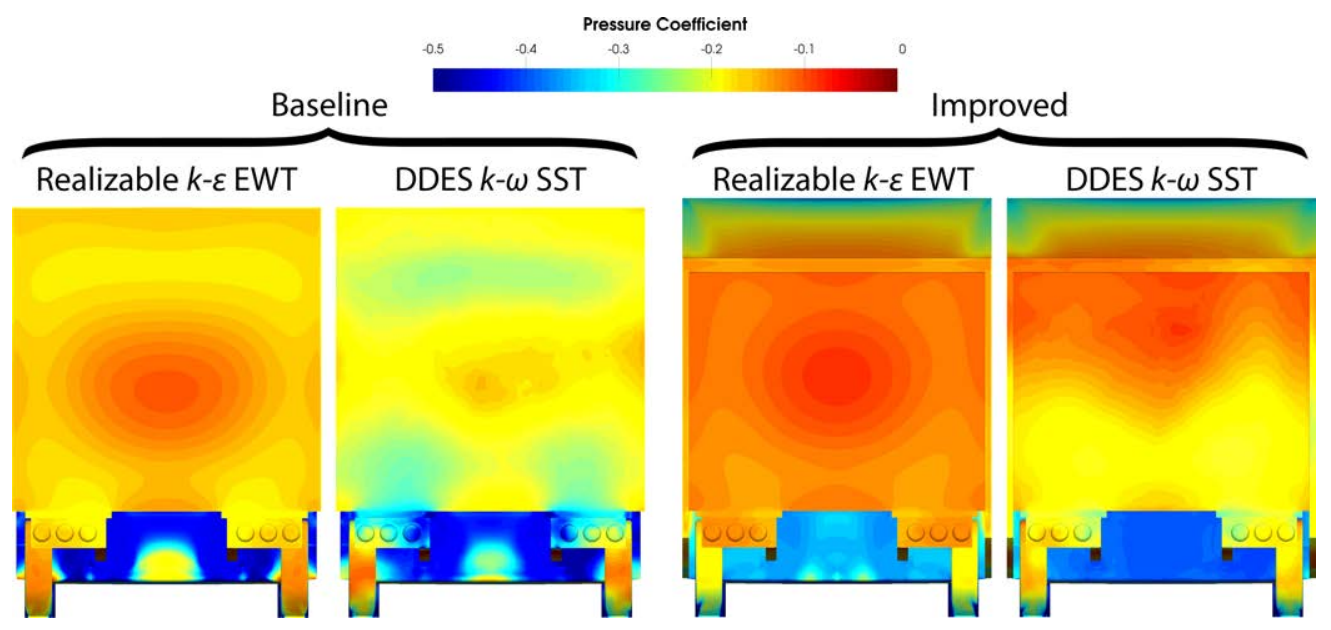

Figure 35: Comparison of the time-average base pressure for the baseline and improved concept with loading volume of $14.7 \mathrm{~m}^{3}$ simulated with the realizable $k-\varepsilon$ EWT and DDES $k-\omega$ SST models. The realizable $k-\varepsilon$ EWT model captures most of the high-pressure regions well, while significantly missing the magnitude of the low-pressure regions, compared to the DDES $k-\omega$ SST model. 


\section{Important Factors for Accurate Scale-Resolving Simulations of Automotive Aerodynamics}

The light truck cab, chassis, and roof air deflector is assumed to be fixed in the study, thereby is only modifications to the swap body allowed. In the investigation of aerodynamic concepts, affecting the swap body, the available loading volume inside the swap body is of high priority, to ensure a practical operation solution. The usable loading volume is conservatively decided as a function of the length, width and the lowest height inside the swap body. Adding skirts and improving the front part of the swap body by aligning the front part with the roof air deflector and $30 \mathrm{~mm}$ radius on the side of the swap body results in a significant reduction of drag with only a small loss to the load volume, Figure 33. This removed separations, thereby significant total pressure losses, from the front top part of the swap body, Figure 34. Streamlining the top part of the swap body resulted in further, but not as significant, reduction of drag but with a higher cost of load volume. This resulted in less pressure losses for the rear wake and thereby increasing the base pressure, Figure 34 and 35.

To ensure that the drag reduction predictions from the RANS simulations are valid and sufficiently accurate, as RANS rarely can offer reliable accuracy, two simulations with the DDES $k-\omega$ SST are performed. Similar percentual reduction of drag is seen for the RANS (33.3\%) and DDES (35.5\%) models for the baseline and concept with 14.7 $\mathrm{m}^{3}$ load volume, respectively. However, the absolute values are significantly different and almost show 50 drag counts $\left(\Delta C_{D}=0.050\right)$ difference between the models. Investigation of the total pressure losses, Figure 34, shows similar behavior of the overall total pressure losses. However, the magnitude of the total pressure is different between the turbulence models in specific regions, e.g. in the wake, causing significant differences for the base pressure, Figure 35. Significantly higher pressure is seen for the realizable $k-\varepsilon$ model compared to DDES $k-\omega$ SST. The realizable $k-\varepsilon$ EWT model capture most of the high-pressure regions well while missing, especially the magnitude of the lower pressure regions, seen for the DDES $k-\omega$ SST model. This results in a severe difference between the models of the $\Delta C_{D}$ for the base between the baseline and improved light truck, where a $40\left(\Delta C_{D}=0.040\right)$ and $65\left(\Delta C_{D}=0.065\right)$ drag counts reduction are seen for the RANS and DDES models, respectively.

From the investigation, a prototype swap body was manufactured (corresponding to the blue square in Figure 33) and tested for eight months. From the testing period, a fuel reduction of $12 \%$ was reported, corresponding to a $24 \%$ reduction of drag by the rule of thumb (where half the percentage of reduced drag equals the percentage reduced fuel consumption), being in reasonable agreement with the predicted drag reduction of $29 \%$.

The drag from the base is a significant part of the drag from timber trucks, especially when unloaded. In Sweden, around 2000 timber trucks exist, which have more than $30 \%$ higher fuel consumption than tractor-trailer combinations [90]. No encouraging improvement of the fuel consumption has been seen during the last 20 years, and one of the main reasons behind it can be the lack of improvement of the aerodynamic drag.

In Paper IV, an investigation of a straight boat tail-like shield is fitted to the bulkhead of an unloaded timber truck to reduce drag, Figure 36 panel a. The device is meant to reduce the pressure losses in the wake, thereby increasing pressure on the base without compromising the loading procedure and the loading capacity of the vehicle. This, together with high interaction between wake from the cab and downstream components like bunks 


\section{A Reality Check: Drag Reduction}

and stakes, makes RANS unreliable for accurate predictions of the drag change, Figure 36 panel b. Therefore, the SBES $k-\omega$ SST DSM model is used in this study.
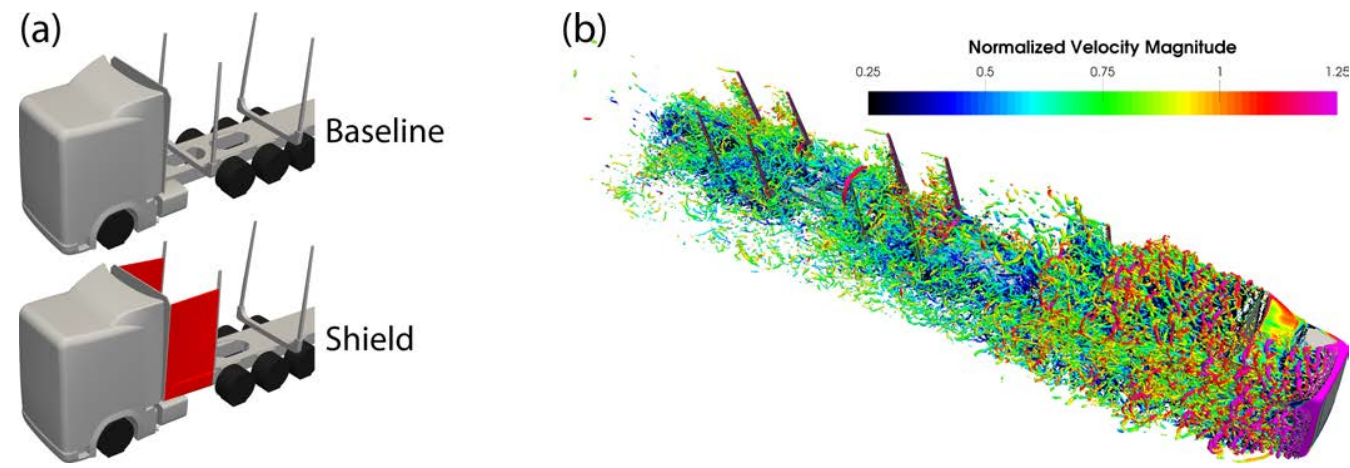

Figure 36: (a) Geometry of the front part of the unloaded timber truck with and without the drag reduction shield. The shield extends from the bulkhead to the first stake pair to not disturb the operating of the truck. (b) The flow around timber trucks is highly complex and involves much flow interaction, as seen for the coherent turbulent $\left(Q=5 \cdot 10^{5} \mathrm{~s}^{-2}\right)$ structures at $5^{\circ}$ yaw.

In Figure 37, the static pressure is seen to be low around the stakes and in the two large counter-rotating vortices for the baseline configuration. This results in lower pressure on the base surface of the bulkhead, seen in Figure 38. By adding the drag reduction shield, the lower pressure around the stakes is removed, weakening the counter-rotating vortices in the wake and resulting in a pressure increase of the base. The shield results in a drag reduction for all three investigated yaw angles $\left(0^{\circ}, 5^{\circ}\right.$ and $\left.10^{\circ}\right)$, Table 4 .

Table 4: The drag coefficient for the baseline and drag reduction shield configurations at $0^{\circ}, 5^{\circ}$ and $10^{\circ}$ yaw. Significant drag reduction is seen when adding the shield for yaw angles up to $5^{\circ}$, and only a small benefit is seen for $10^{\circ}$ yaw.

\begin{tabular}{lccc} 
Configuration & $0^{\circ}$ & $5^{\circ}$ & $10^{\circ}$ \\
\hline Baseline & $C_{D}=0.665$ & $C_{D}=0.740$ & $C_{D}=0.872$ \\
Shield & $C_{D}=0.620$ & $C_{D}=0.713$ & $C_{D}=0.863$ \\
\hline$\Delta C_{D} \cdot 1000$ & -45 & -37 & -9 \\
\hline
\end{tabular}

The drag reduction is mainly achieved from the front part of the timber truck, where the shield is located. However, the components downstream (bunks, stakes and wheels) see an increase of drag as an effect of the reduced wake. This is due to more flow with high energy impinging these components, showing the possibility for further gains with modifications to the vehicle. 
Important Factors for Accurate Scale-Resolving Simulations of Automotive Aerodynamics
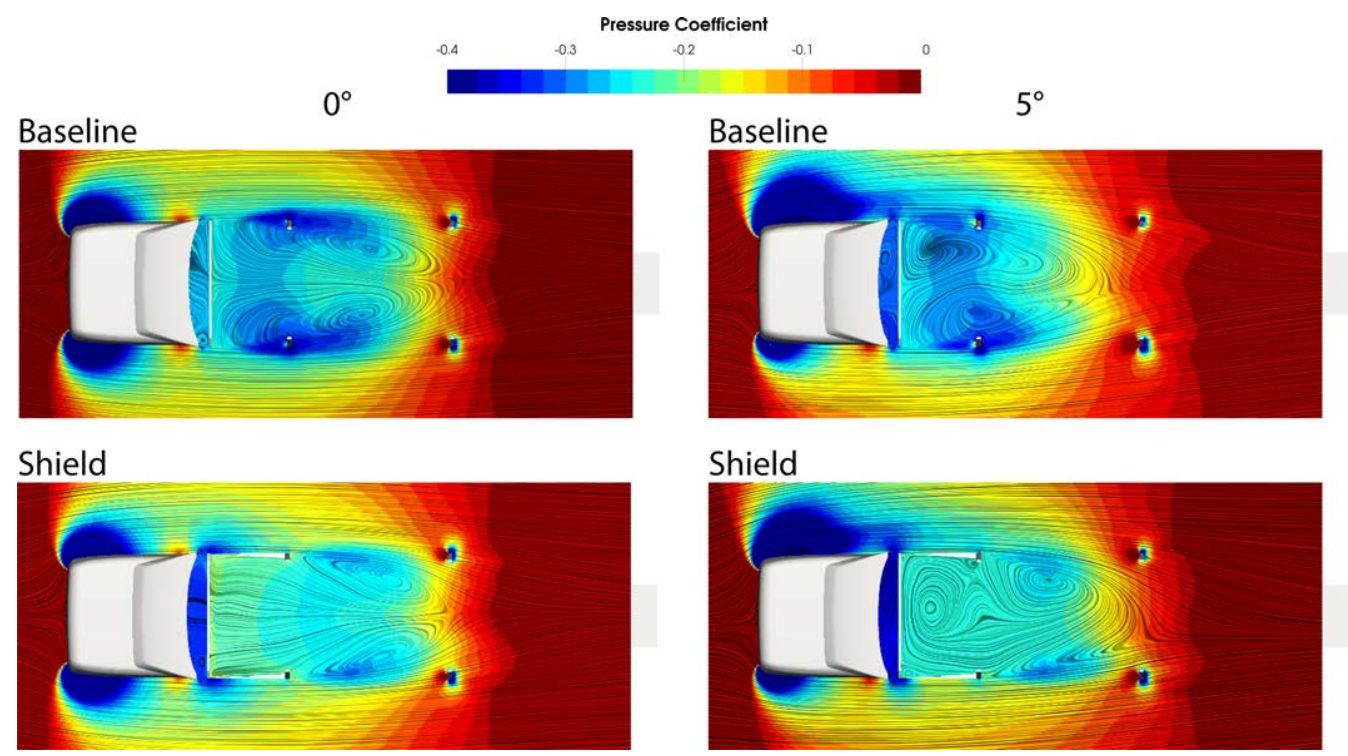

Figure 37: Time-averaged pressure coefficient and streamlines at a plane of $40 \%$ height of the vehicle for the baseline and drag reduction shield configurations at $0^{\circ}$ and $5^{\circ}$ yaw. The wake mainly consists of two counter-rotating vortices with low pressure, which are weakened and moved further downstream when adding the drag reduction shield.

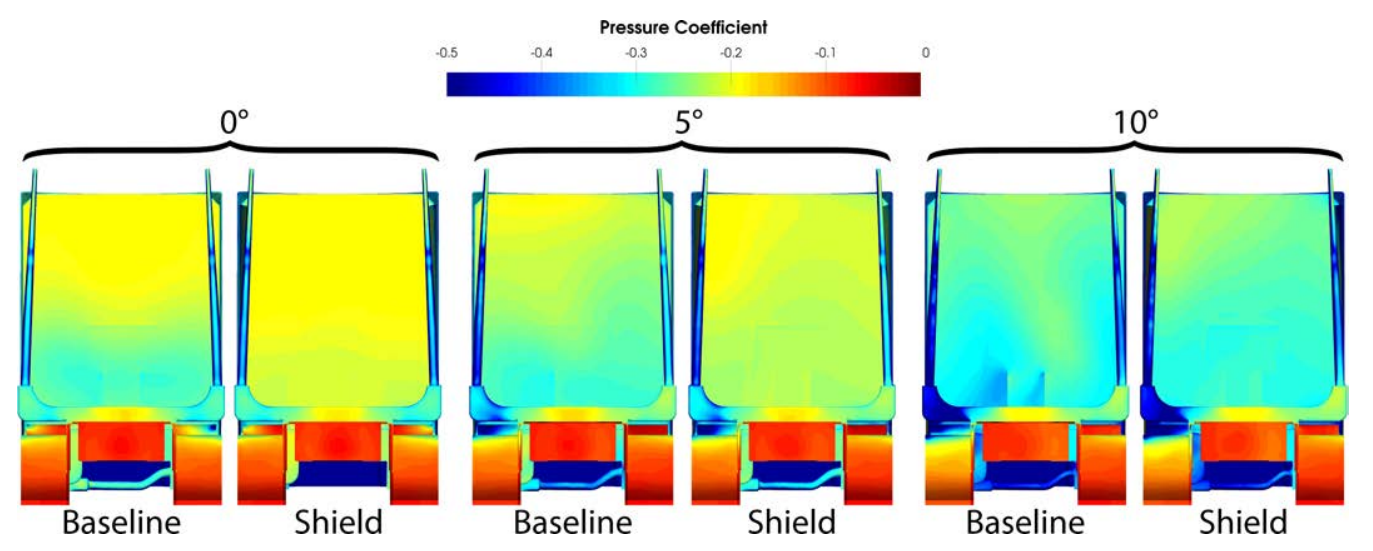

Figure 38: Time-averaged pressure coefficient for the base of the baseline and drag reduction shield configurations at $0^{\circ}, 5^{\circ}$ and $10^{\circ}$ yaw. Significant pressure increase is seen for the lower part of the bulkhead when adding the shield. 


\subsection{Reducing Computational Cost}

The previous scale-resolving simulations are performed with a fine temporal resolution, following best practice guides [45, 64, 69] of keeping the maximum CFL value below unity in the LES region. This results in that many ten thousand, and even several hundred thousand time-steps, are needed for achieving a properly time-averaged solution. With between three to five inner loop iterations per time steps needed, even more iterations are necessary. This can be compared with RANS, where efficient solvers today needs between 200-500 iterations to achieve a well-converged solution. With increasing needs of virtual development and higher accuracy, e.g., to meet the WLTP requirements, faster and more accurate simulations are of high need.

Reducing the computational cost can be performed with more efficient numerics, faster hardware, coarser mesh resolution and larger time-steps. The first is very software dependent, and CFD software is continually getting faster and more efficient. Changing/improving software and increasing hardware resources can often be difficult and expensive, leaving the mesh and time-step resolution as the only real candidates. Reducing the mesh resolution is an efficient way to reduce the computational cost, as it does not only affects the computational cost per iteration but also makes it possible to increase the time-step size (and still following best practice guides), as the CFL value is a function of the local cell size. However, reducing the mesh resolution may not always be possible, as the geometrical representation and accuracy most often suffer. Few studies exist in the literature on the effects of increasing the time-step size for the hybrid RANS-LES methods.

In Paper $\mathrm{V}$, the effects of changing the time-step size is investigated for the generic car body DrivAer at $5^{\circ}$ yaw. The notchback rear-end configuration is chosen, as a complex flow over the rear window is seen in wind tunnel measurements performed at TU Berlin [65, 66]. For accurate comparison with these measurements, a significant part of the wind tunnel is included in the simulation domain, Figure 10 panel c and Figure 11 panel a. The smallest investigated time-step size ensures that only 595 cells within the LES region (corresponding to $5.65 \cdot 10^{-4} \%$ of the full mesh) do not follow the $C F L_{\max } \leq 1$ criterion, and that 20850 time-steps are needed for a single flow unit. Four larger time-step sizes are investigated to see the effects on computational cost and accuracy. All simulations were run for the same physical time, which ensured the moving mean (time-averaged) drag coefficient to fluctuate less than half a drag count $\left(\Delta C_{D} \pm 0.0005\right)$. To ensure convergence of the instantaneous solution, at least three inner loop iterations together with the requirement to reach below $10^{-5}$ for the normalized residuals is required.

Significant reduction in simulation time (and hence computational cost) is achieved when increasing the time-step size, Figure 39 panel a. Time-step size factor $50(\Delta t \cdot 50$, notated CFL50 in Paper V) only takes $3.9 \%$ of the simulation time compared to the smallest investigated time-step size $(\Delta t \cdot 1)$. Slightly longer simulation time is seen for the timestep factor $100(\Delta t \cdot 100)$, as a result of needing more inner loop iterations per time-step. Only small differences $\left(\Delta C_{D} \leq 0.001\right)$ is seen for the drag when increasing the time-step up to a factor of $20(\Delta t \cdot 20)$, while slightly larger difference and increasing trend is seen for the larger time-step sizes, Figure 39 panel b. For time-step sizes up to $\Delta t \cdot 50$, the predicted drag is in good agreement with the measurements [65], with less than one drag 


\section{Important Factors for Accurate Scale-Resolving Simulations of Automotive Aerodynamics}

counts difference to the measurement error range.

More substantial differences are seen for the lift force when changing the time-step size, Figure 39 panel c, in agreement with [91]. Small effects are seen for the front lift force $\left(C_{L f}\right)$, as the changes mainly occur for the rear lift force $\left(C_{L r}\right)$, with the same behavior seen for a similar study [92]. The best correlation to $\Delta t \cdot 1$ is seen for $\Delta t \cdot 100$, which is a result of pressure differences canceling each other on both the top and bottom side of the body. A more considerable difference to the measurement is seen for the lift force (17 lift counts), which is difficult to predict accurately and more sensitive to the car body set-up in the test-section.
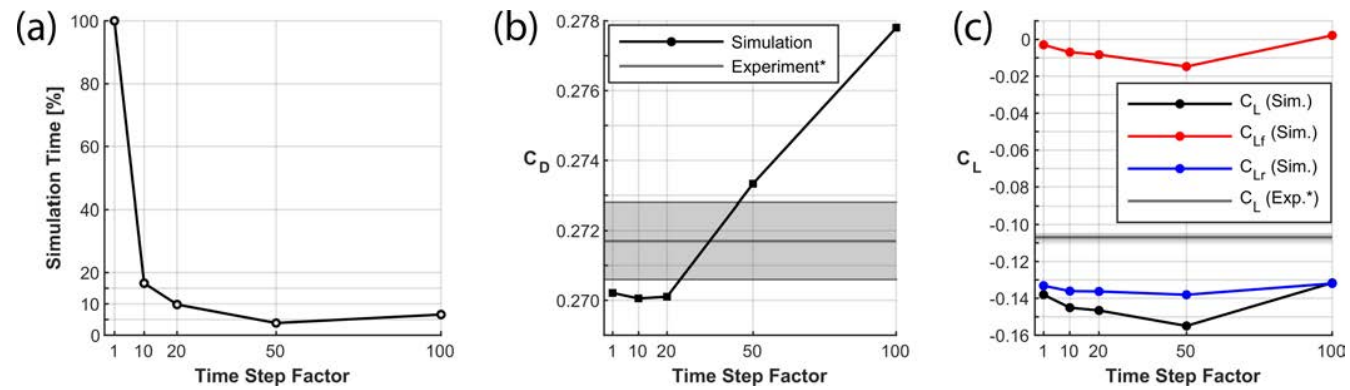

Figure 39: (a) A significant reduction in simulation time is achieved with increased time-step size. (b) Relative small differences are seen for the time-averaged drag when changing the timestep size, especially up to $\Delta t \cdot 20$. (c) Good agreement to wind tunnel measurements (where grey region indicate measurement error) [65] are for time-step sizes up to $\Delta t \cdot 50$, while less agreement is seen for the lift force. Higher sensitivity is seen for the rear lift force when changing the time-step size. The results are for the DrivAer notchback car body $5^{\circ}$ yaw. Note the different scales for $C_{D}$ and $C_{L}$.

The difference for the accumulated drag is seen to mainly originates from the wheels, mirror/A-pillar region, rear window and the base, Figure 40 panel a, all being highly unsteady regions. As for the full-body drag, only small differences are seen when increasing the time-step size with a factor up to 20. A similar trend is seen for the accumulated lift force along the car body, Figure 40 panel b. Here, the differences mainly occur from and downstream the mirror/A-pillar region, explaining the sensitivity of $C_{L r}$ in Figure 39 .

Pressure is the dominating part of the total force acting on a vehicle, therefore explaining where the differences of the forces originate from is of importance. In Figure 41, the time-averaged pressure difference to the $\Delta t \cdot 1$ is compared for the four larger time-step sizes. An increase of the pressure difference is seen when increasing the time-step size, with differences mainly originating from the wheels, mirror/A-pillar region, rear window and base, being regions where differences of the drag and lift forces are seen.

Especially at the wheels and mirror/A-pillar region, the small turbulent structures with small time-scales cannot be captured with larger steps in time and causing slight differences to the flow field. This results in a different behavior for the A-pillar vortices for the larger time-step sizes as they reattach later on the side window, which is seen by the existence of both low and high-pressure differences. The A-pillar vortices affect the flow over the rear 

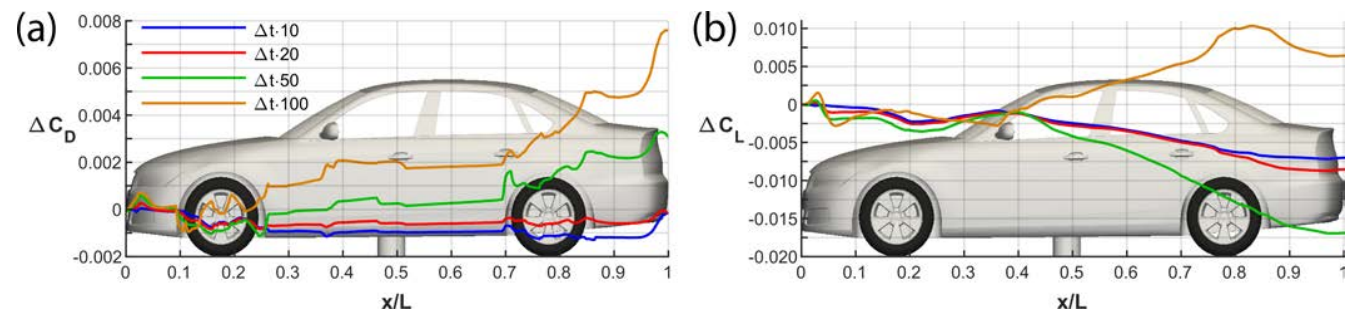

Figure 40: Accumulated drag (a) and lift force (b) differences for the larger time-step sizes against the $\Delta t \cdot 1$ time-step size for the DrivAer notchback car body $5^{\circ}$ yaw. Higher sensitivity to the time-step size is seen for the lift force, as larger difference occurs even for $\Delta t \cdot 10$. Reprinted from Paper IV with permission from SAE International.

window significantly and are partly the reason for the complex near-wall flow at the rear window. With these vortices slightly differently captured, which for the larger time-step sizes achieve a slightly different trajectory, this causes pressure differences on the rear window and trunk. This is the reason for the lift force difference, especially for $\Delta t \cdot 50$ and $\Delta t \cdot 100$. Overall, only small pressure differences are seen for $\Delta t \cdot 10$ and $\Delta t \cdot 20$, with only more considerable differences seen at the wheels, which indicate the importance of fine temporal resolution for studies of these regions.

Similar behavior, as seen for the surface pressure, is seen for the skin friction when increasing the time-step size, Figure 42. However, a general increase of the skin friction occurs with larger time-steps, as an effect of changing the behavior of the separations. Larger time-step sizes result in longer transport of the flow field per time-step and can lead to a more diffusive transportation of flow field information. This can be of high importance for separating flows and especially for the initialization of the scale-resolving when transition from the RANS to the LES region in Hybrid RANS-LES methods. However, different behavior for different separation characteristics are seen. Smaller and/or less separation is seen for regions with strong separations, e.g. at the windward rear wheel. Increased size for weaker separations is seen, typically occurring at curved surfaces, as seen for the windward a-pillar vortex. Only some slight differences in the near-wall flow structures at the rear window is seen in Paper V, as slightly smaller recirculation regions occur at the rear window for the larger time-step sizes (blue isosurface in Figure 42). No significant changes to the rear wake are seen for the outer region of the recirculating flow.

Less of the smaller turbulent structures are seen when increasing the time-step size, Figure 43, where the instantaneous Q-criterion is colored with the CFL value. Especially for the A-pillar vortices, wakes of the mirrors and wheels larger time-steps results in diminishing of the smaller turbulent structures. The time-step $\Delta t \cdot 100$ is seen to break the CFL $\leq 1$ criterion for almost all regions, and values below unity can only be seen in the central section of the rear wake. The flow field information, therefore, convects many cells for each time-step, resulting in more diffusive behavior, and suppression of all turbulent structures of higher frequencies than the time-step size $(\Delta t \cdot 100 \approx 7142 \mathrm{~Hz})$. For $\Delta t \cdot 20$, much more of the resolved turbulent structures are within the CFL $\leq 1$ criterion, with only some exceptions in the regions with strong flow acceleration and mesh refinements (e.g. 


\section{Important Factors for Accurate Scale-Resolving Simulations of Automotive Aerodynamics}
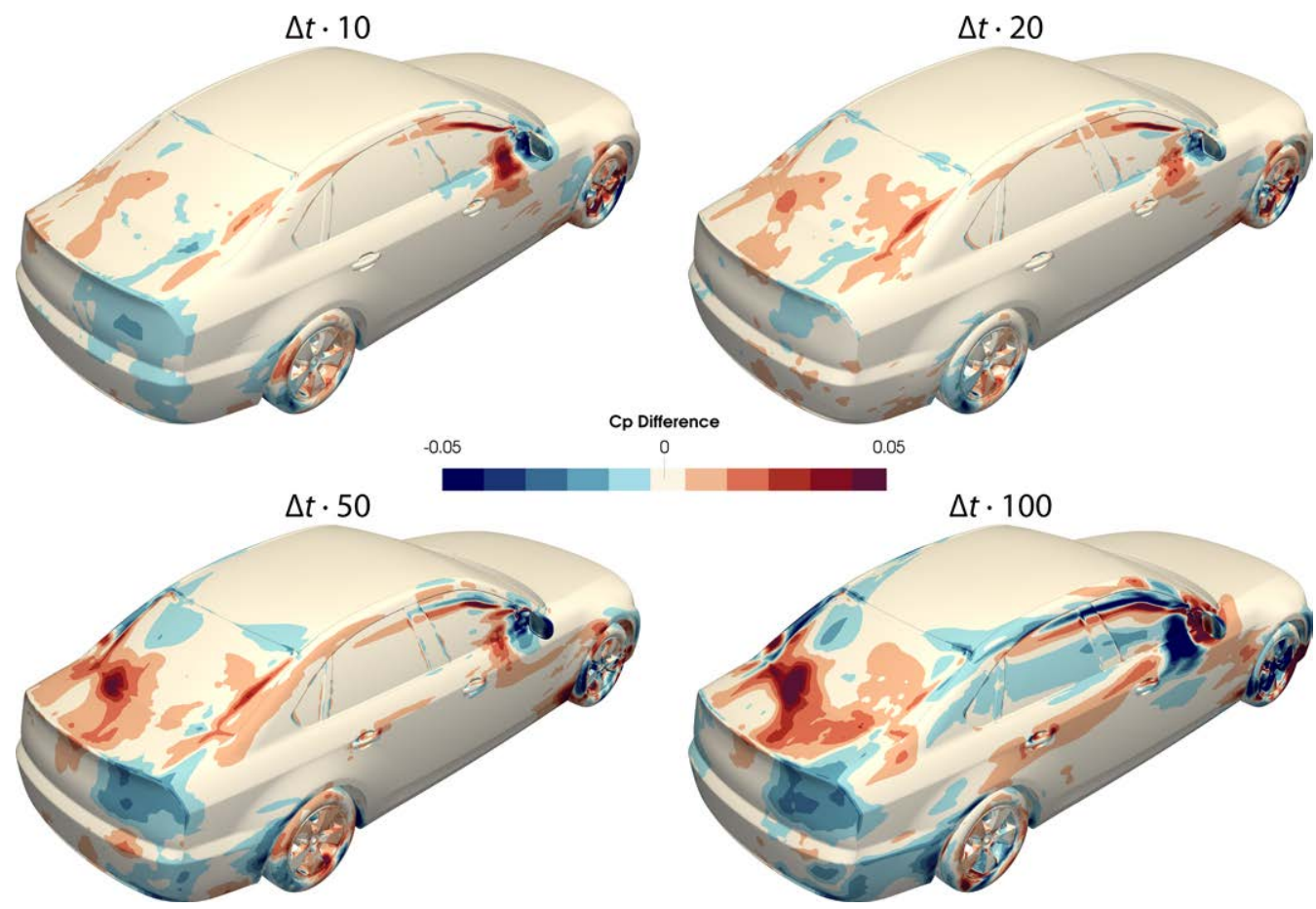

0.05

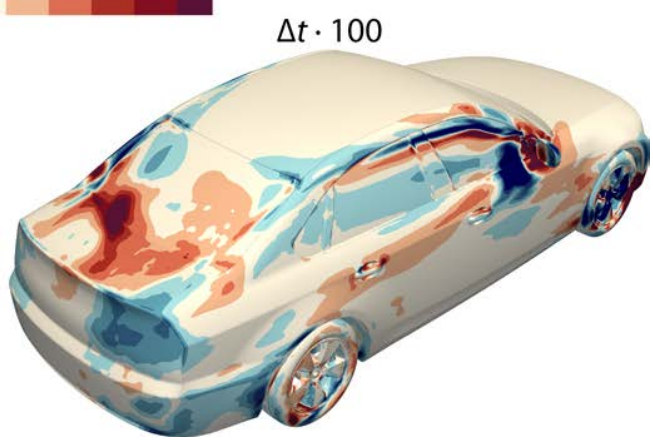

Figure 41: Pressure differences for the larger time-step sizes against the $\Delta t \cdot 1$ time-step size for the DrivAer notchback car body $5^{\circ}$ yaw. Note the small pressure coefficient difference range, for easier distinguishing of the differences. Pressure differences are seen to mainly arise from the unsteady regions, e.g. wheels and mirrors.

A-pillars, mirrors, C-pillars and the wheels). Almost no differences in terms of size and locations are seen for the turbulent structures for the time-step $\Delta t \cdot 20$ when compared to $\Delta t \cdot 1$, indicating that most of the turbulent time-scales are lower than $35714 \mathrm{~Hz}$. For $\Delta t \cdot 1$, the turbulent structures are well within the CFL $\leq 1$ criterion in almost the whole domain, as only 595 cells violate it.

The time-step size has rather small effects on the time-averaged forces but seemingly affect the resolved structures and might, therefore, affect and possibly reduce pressure fluctuations. To see this effect on the forces, the RMS drag and lift forces are calculated from the RMS pressure on the car surface. The car body is divided into 500 sections, where each sections drag and lift force RMS is visualized in Figure 44. Only the $\Delta t \cdot 1$ and $\Delta t \cdot 100$ is shown for clarity, as the other time-step showed minor difference to $\Delta t \cdot 1$. Similar behavior of the RMS forces is seen between the time-step sizes, with only differences to the magnitude of the fluctuations. For the RMS drag, mainly these magnitude differences are seen for the front and rear wheelhouses and rear window. More significant differences are seen for the RMS lift, and especially at the front bumper and rear window. This might be important to consider for studies of aerodynamic stability, as it might cause magnitude changes of the instantaneous $C_{L f}$ and $C_{L r}$. 

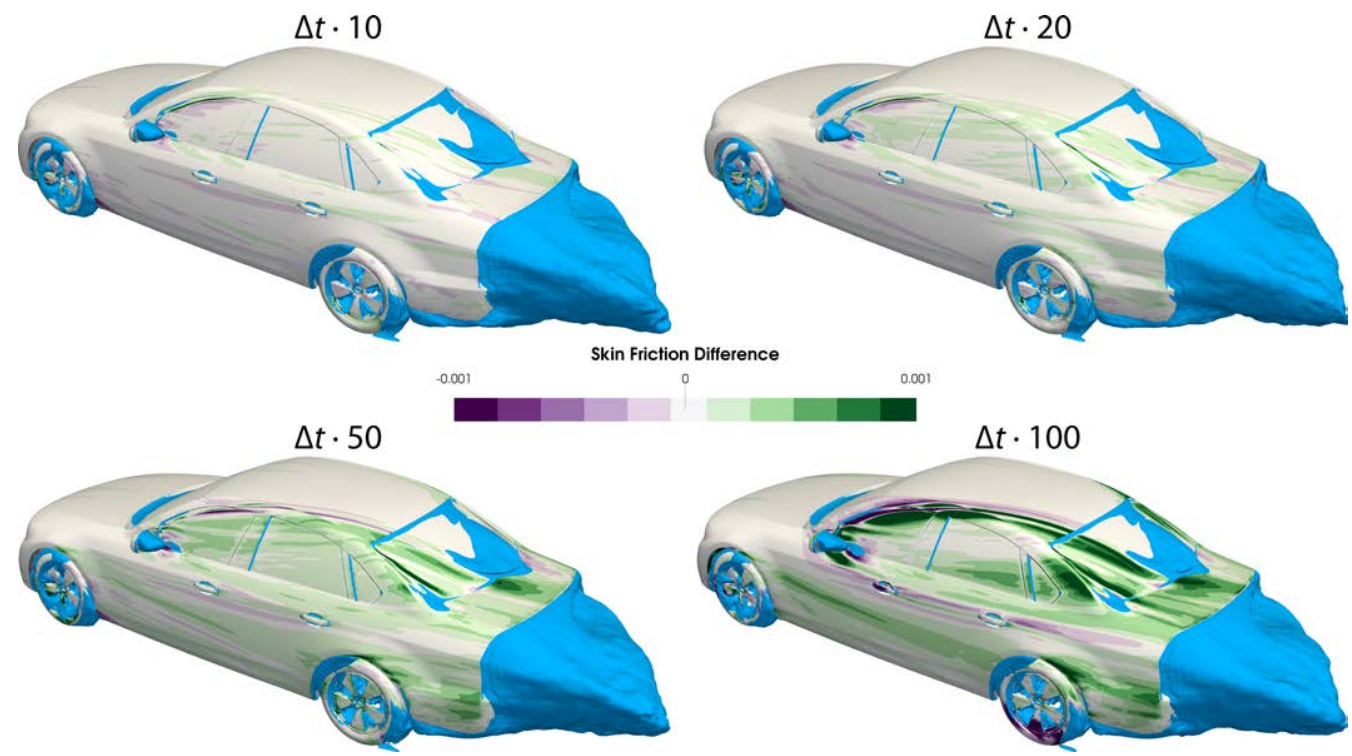

Figure 42: Generally, higher skin friction occurs compared to the $\Delta t \cdot 1$ time-step size when increasing the time-step size for the DrivAer notchback car body $5^{\circ}$ yaw. Only some slight differences are seen for the recirculating flow with increased time-step sizes (shown with the blue isosurface equaling $x$-velocity equal to zero), especially around the windward rear wheel.

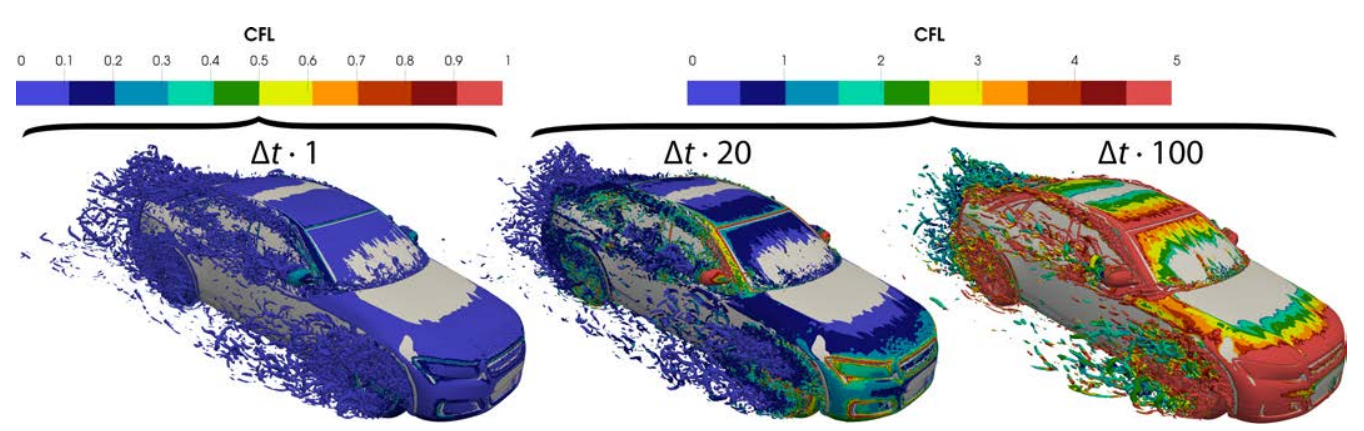

Figure 43: Visualization of the coherent turbulent structures $\left(Q=3 \cdot 10^{6} \mathrm{~s}^{-2}\right)$ colored with the CFL value for three time-step sizes for the DrivAer car body $5^{\circ}$ yaw. Note the different colorbar range for $\Delta t \cdot 1$ and the larger time-step sizes. Significantly less small turbulent structures are seen for $\Delta t \cdot 100$, while the similar size of the turbulent structures is seen for $\Delta t \cdot 1$ and $\Delta t \cdot 20$.

With less resolved turbulent structures, effects on the resolved turbulent kinetic energy is to be expected, at least for regions with high CFL values. In Figure 45, the resolved turbulent kinetic energy is compared at the $y=0$ plane and RMS pressure at the car surface for $\Delta t \cdot 1, \Delta t \cdot 20$ and $\Delta t \cdot 100$. No significant difference between the time-step sizes can be seen for the resolved turbulent kinetic energy in the wake, as the smaller turbulent 

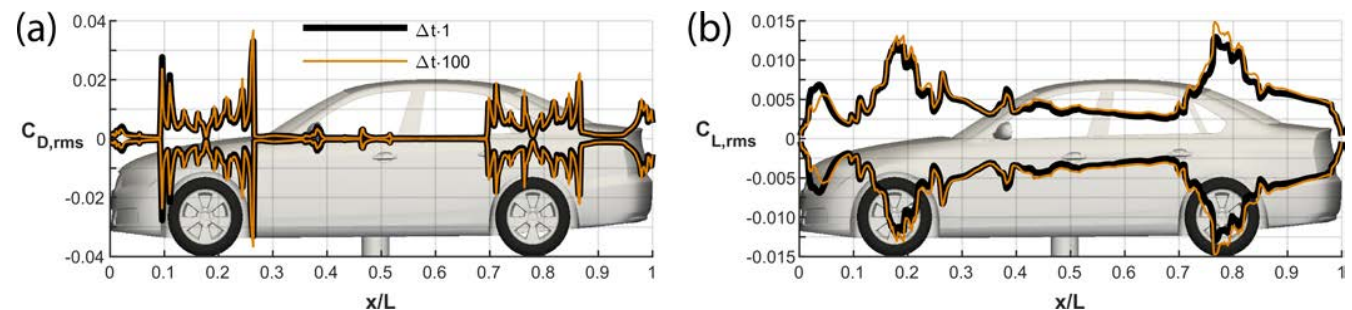

Figure 44: RMS drag (a) and lift (b) pressure forces for each section of the DrivAer notchback car body at $5^{\circ}$ yaw for $\Delta t \cdot 1$ and $\Delta t \cdot 100$. Similar behavior is seen for the two time-step sizes, where only differences to the magnitude occur for some of the most unsteady regions.

scales not represent a significant part of the turbulent kinetic energy. For the rear wake, even the $\Delta t \cdot 100$ time-step size is within, or at least close to, the CFL $\leq 1$ criterion, and therefore reasonably well-resolved in time. However, for regions less resolved in time (higher CFL values), e.g. mirrors, more significant differences are seen for the resolved turbulent kinetic energy, Figure 46. Here the resolved turbulent kinetic energy is occurring much later for $\Delta t \cdot 100$ than for $\Delta t \cdot 1$, as it takes a longer distance to generate the resolved turbulence, causing a more downstream high RMS pressure on the side window. Only small differences to $\Delta t \cdot 1$ is seen for $\Delta t \cdot 20$, suggesting that this time-step size is enough for capturing even small details of the flow field.

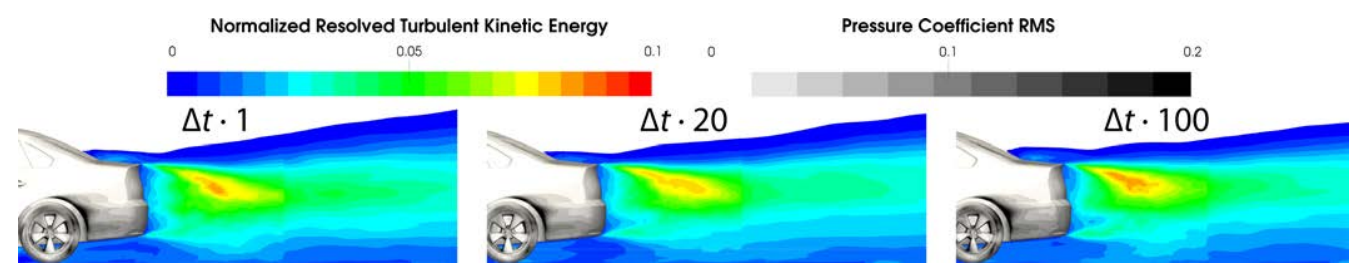

Figure 45: Resolved turbulent kinetic energy for the wake of the DrivAer notchback car body at $5^{\circ}$ yaw for three time-step sizes. No significant differences are seen between the timestep sizes, as even the $\Delta t \cdot 100$ is reasonably well-resolved in time for this region. No significant differences are either seen for the pressure coefficient RMS of the car surface for the time-step sizes.

It should be noted that the default under relaxation settings (see Paper $\mathrm{V}$ for details) for the SIMPLEC solver is used for the time-step size investigation. Therefore, there exist great possibilities to reduce the computational cost even more. Optimizing the solver relaxation settings is outside the scope of Paper $\mathrm{V}$, as these settings can be software dependent. Using a coupled solver may also reduce possible convergence instabilities when using larger time-step sizes. However, no difference in accuracy and trends are expected with a different solver scheme or relaxations, as the temporal resolution causes the differences. Tests were performed with the pressure-velocity coupled solver for the $\Delta t \cdot 20$ time-step size, resulting in less than a drag count difference. 


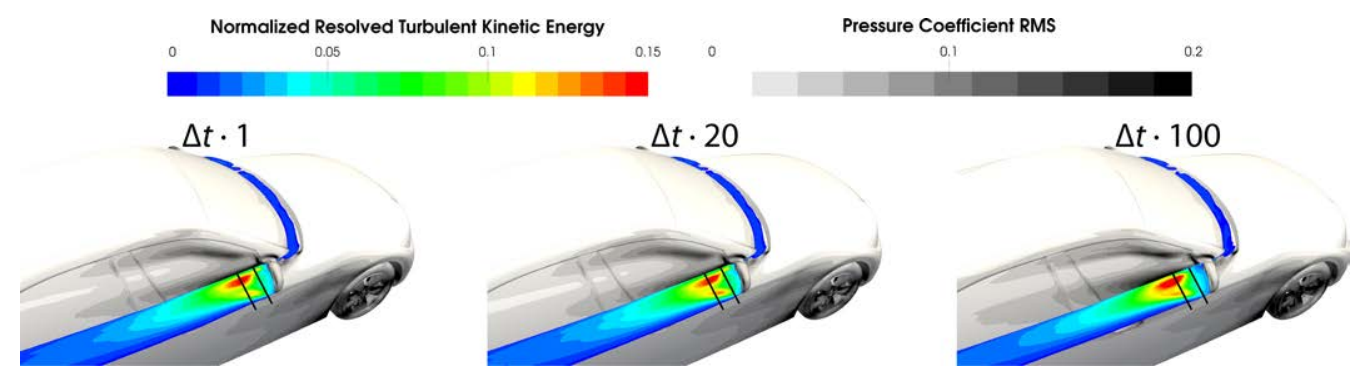

Figure 46: Resolved turbulent kinetic energy for the leeward mirror region of the DrivAer notchback car body at $5^{\circ}$ yaw for three time-step sizes. Significant differences are seen between time-step sizes, as the resolved turbulent kinetic energy occurs much further downstream for $\Delta t \cdot 100$ compared to $\Delta t \cdot 1$, resulting in a higher RMS pressure coefficient on the side window for $\Delta t \cdot 100$. Black lines are support lines for easier identification of where the scale-resolving starts.

\subsection{Reliable Accuracy: Hybrid RANS-LES Models}

The SBES $k-\omega$ SST DSM, together with the time-step size $\Delta t \cdot 20$, seems to be a reasonable compromise between accuracy and computational cost. To ensure that reliable accuracy is achieved, it is investigated and compared against wind tunnel measurements for both the notchback and fastback DrivAer configurations for yaw angles up to $7^{\circ}$. This to see if the SBES $k-\omega$ SST DSM model is an improvement compared to both DDES $k-\omega$ SST and IDDES $k-\omega$ SST, as these models have shown good correlation to measurements $[93,94,95,96,97,98]$ and is stated as best practice for some commercial software's for automotive aerodynamic simulations.

Reasonable agreement for the investigated models to the measurements is observed for the drag force of the notchback configuration during the yaw sweep, Figure 47 panel a. Both the DDES and IDDES models struggle for consistent agreement with the measurements over the yaw sweep. In contrast, the SBES model is consistent with the measurement and within the error range throughout the yaw sweep. This results in a high accuracy prediction of the $\Delta C_{D}$ for the SBES model when changing the yaw angle. This is especially important with the introduction of WLTP certification of the CFD methodology, as high accuracy of changes, although geometrical, needs to be demonstrated for approval of the certification. Less agreement with the measurements is seen for the lift force for all the models, Figure 47 panel b. The DDES model is closest to the measurement for the absolute values but is not able to follow the convex behavior of the lift force seen in the measurement. The IDDES and SBES models (except for $2^{\circ}$ yaw) capture the convex shape and do for the higher yaw angles achieve similar $\Delta C_{L}$ against $0^{\circ}$ yaw, see Figure 3 in Paper VI.

Investigating the accumulated drag and lift forces, Figure 48, show very similar behavior for the investigated models, as only significant differences exist for the rear part of the car body $x / L \geq 0.7$. Some slight differences for the lift force are also seen for the front part of the body as a result of slightly different predicted separation for the flow around the bottom lip of the front bumper. This is a sensitive region for the DrivAer car body, 


\section{Important Factors for Accurate Scale-Resolving Simulations of}

Automotive Aerodynamics

(a)

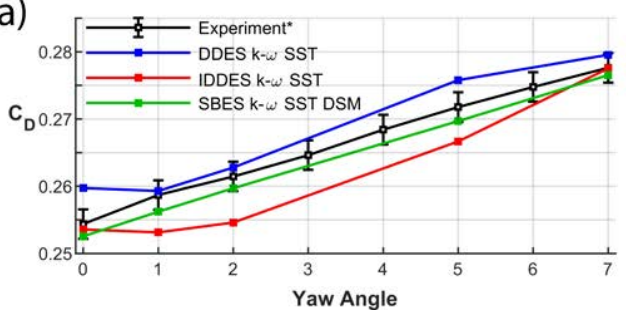

(b)

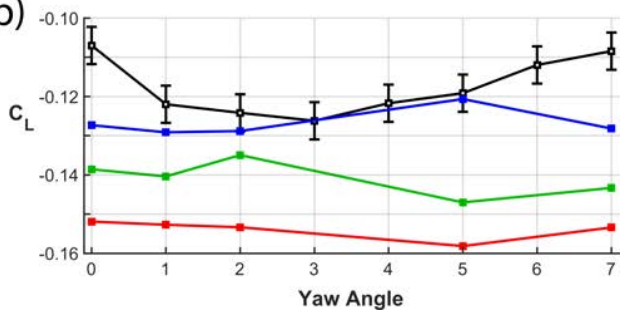

Figure 47: The drag (a) and lift (b) force coefficients for the investigated hybrid RANS-LES models and wind tunnel measurement [65] for the notchback DrivAer body for a yaw sweep. Generally, good agreement to the measured drag force is seen for the models, where particularly the SBES $k-\omega$ SST DSM is consistently close to the measurement. More substantial differences are seen for the lift force, where the DDES $k-\omega$ SST is in best agreement with the measured lift force.

where many turbulence models predict different separation size and pressure distribution [99]. Seen in Figure 13 in Paper VI, similar separation length is seen for the IDDES $k-\omega$ SST and SBES $k-\omega$ SST DSM models, while more significant separation is seen for the DDES $k-\omega$ SST model. The differences between the models are caused by the ability to rapidly transit from the RANS to the LES region (thereby minimizing the gray-area) and the initialization of resolving the turbulence.

(a)

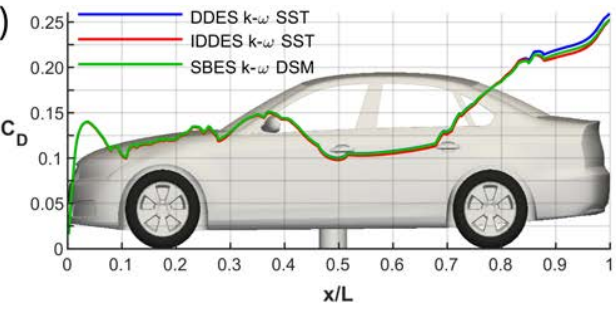

(b)

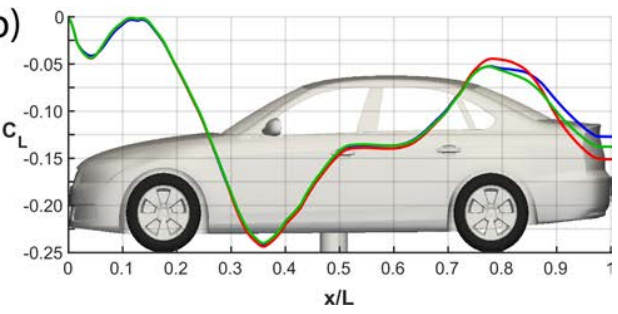

Figure 48: The accumulated drag (a) and lift (b) forces for the three investigated hybrid RANS-LES models for the DrivAer notchback body at $0^{\circ}$ yaw. Mainly differences from the rear part of the body exist, as only slight differences between the models are seen for $x / L<0.7$.

The SBES model manages this transition very well, as more resolved turbulent kinetic energy is present directly from the outer RANS region of the separation point, Figure 49 panel a. The DDES model requires a significantly longer time and distance to achieve the unsteadiness, resulting in less turbulence and a later reattachment. Another effect is also that the RANS shielding fails at the radius of the lip for the DDES model, leading to a LES like model for the near-wall region. As the mesh is not of LES resolution $(\approx 1.5 \mathrm{~mm}$ surface elements), as it is designed for RANS in the near-wall region, this leads to underresolved turbulent structures and Reynolds stresses, thereby GIS. The IDDES model has WMLES possibilities and can, therefore, in this case, manage the collapsed RANS region better. Even when the RANS shielding is sufficient for the DDES model, it cannot achieve the immediate scale-resolving as seen for the SBES model, particularly for the shear layer 
of the lower rear wake, Figure 49 panel b. Shear layers are a common flow feature for vehicles, occurring at separations, the lower turbulent viscosity achieved with Equation 45 , and the $C_{S D E S}$ shear layer tuned constant results in this faster turbulence resolving behavior for the SDES and SBES models, making them well suited for these behaviors. These settings can also be implemented for the DDES and IDDES models but would result in even weaker RANS shielding, as explained in Section 2.3.2, and likely result in even worse results.

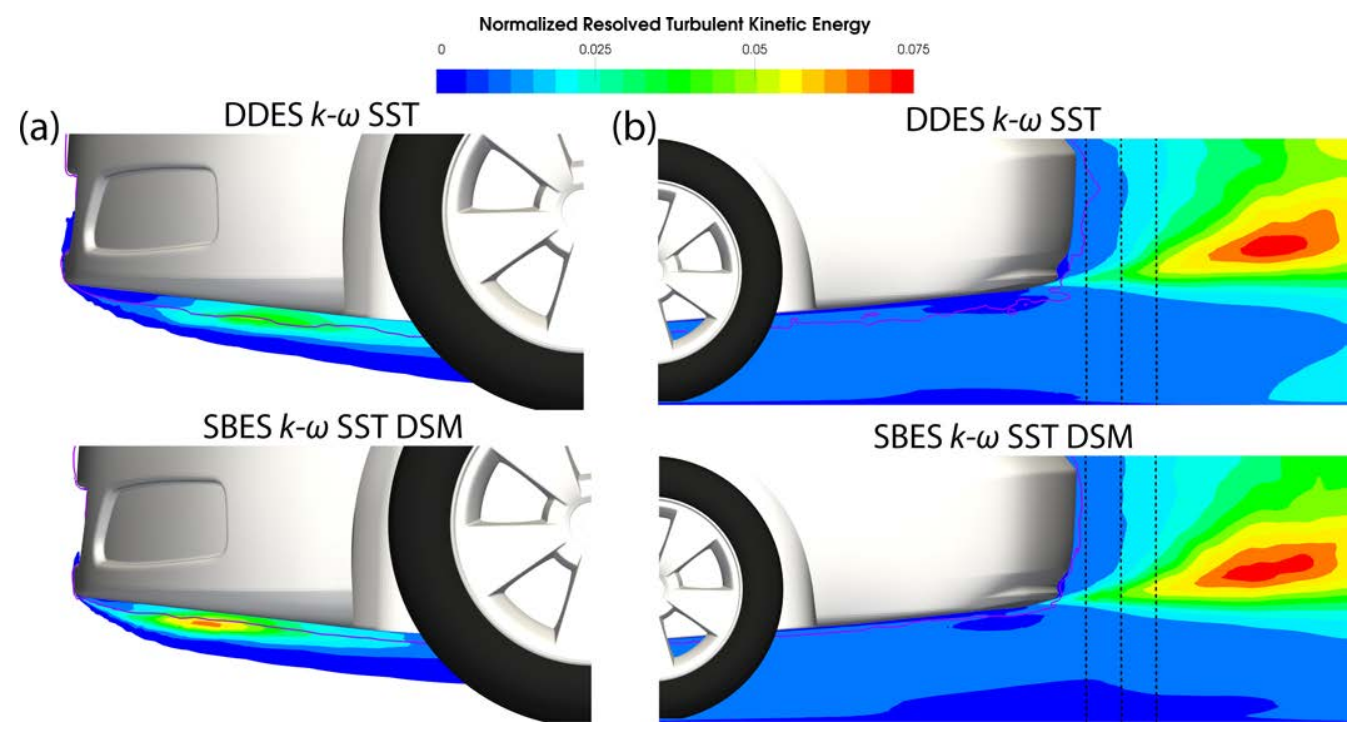

Figure 49: Resolved turbulent kinetic energy at the bottom part of the front bumper (a) and rear bumper (b) for the DDES $k-\omega$ SST and SBES $k-\omega$ SST DSM models, for the DrivAer notchback body at $0^{\circ}$ yaw. The purple line represents the RANS region limit and is seen to fail at the front bumper lip for the DDES $k-\omega$ SST model, as it coincides with the surface of the car. Quicker generation of resolved turbulence kinetic energy is seen for the SBES $k-\omega$ SST DSM model when the flow is separating. Black dashed lines are support lines for easier identification of where the scale-resolving starts.

For the accumulated forces, Figure 48, the differences between the turbulence models mainly originate from the rear part, and especially the rear window, of the DrivAer notchback car body, which also is the region where most differences are seen in the investigation of time-step size, Figure 40. These differences indicate that the flow field over the rear part, and especially the rear window, is a sensitive region of the DrivAer notchback body. Thorough wind tunnel measurements have been performed for investigation of the rear part of the DrivAer car body $[75,65,76,66]$. Oil film visualization over the rear part of the notchback configuration shows complex asymmetric behavior of the near-wall flow, even for symmetric inflow $\left(0^{\circ}\right.$ yaw $)$, Figure 50 and 51 . This is not a specific behavior for the DrivAer notchback body, as it has been observed for other notchback bodies [100, 101, 102]. The rear part of the DrivAer notchback body has some geometrical similarities with the Ahmed body, as the mean rear window angle is $23.4^{\circ}$, being not far from the $25^{\circ}$ slant 


\section{Important Factors for Accurate Scale-Resolving Simulations of Automotive Aerodynamics}

angle of the Ahmed body. However, the lack of sharp edges and more progressive change of the rear window angle results in a significant different flow behavior over the rear window, compared to the flow over the rear slant of the Ahmed body. The presence of the A-pillar vortices, together with the curved C-pillars, results in a more complex flow behavior, which is not dictated and strongly stabilized by the C-pillar vortices as for the Ahmed body. This causes the flow over the rear window of the DrivAer body to be affected more of the upstream flow features and thereby increasing the impact of flow feature interaction, compared to the Ahmed body.

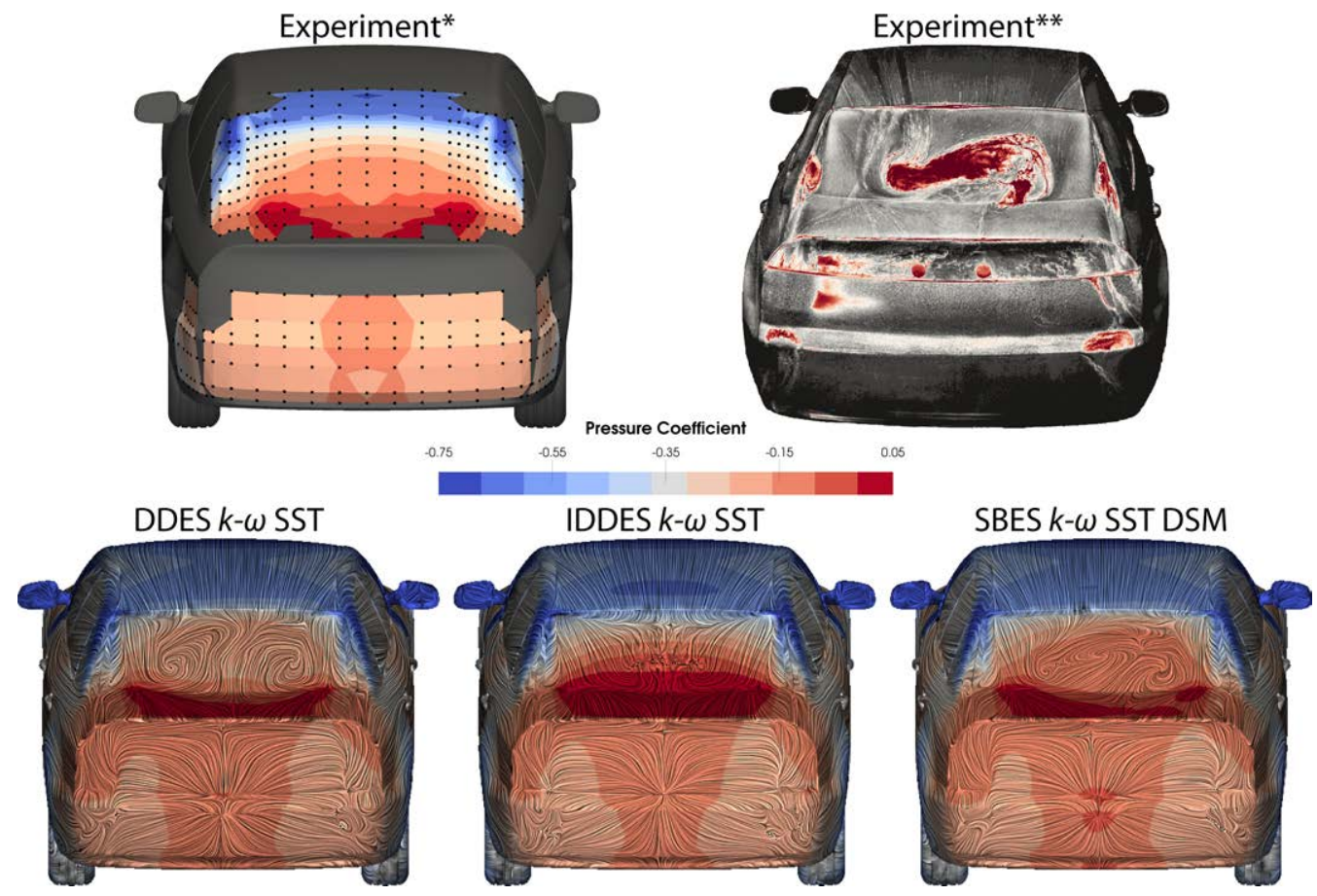

Figure 50: The near-wall flow structures and pressure distribution of the rear of the DrivAer notchback body at $0^{\circ}$ yaw for the measurements, and the three investigated hybrid RANS-LES models. The near-wall flow is achieved from oil film visualization $(* *)$ in the measurement and the wall shear stress field for the simulations. Black points represent pressure probe locations in the measurements. Symmetric pressure distribution is enforced from the measurement $(*)$, as an effect of measurement from only half the body. Good agreement to the measurement for both near-wall flow patterns and pressure distribution is seen for the SBES $k-\omega$ SST DSM model, as it is the only model to predict the complex asymmetric flow structure on the central part of the rear window.

For achieving a finer spatial resolution of the pressure measurements, only half the model was covered with pressure probes (black points in Figure 50 and 51), with some on the other half for references. Pressure measurements were then performed for both positive and negative yaw angles to achieve the full pressure distribution. However, this cannot be performed for $0^{\circ}$ yaw, and therefore a single side measurement is mirrored into symmetric 
pressure distribution. This might neglect any asymmetric behavior of the pressure field, which might be expected due to the asymmetric oil film visualization.

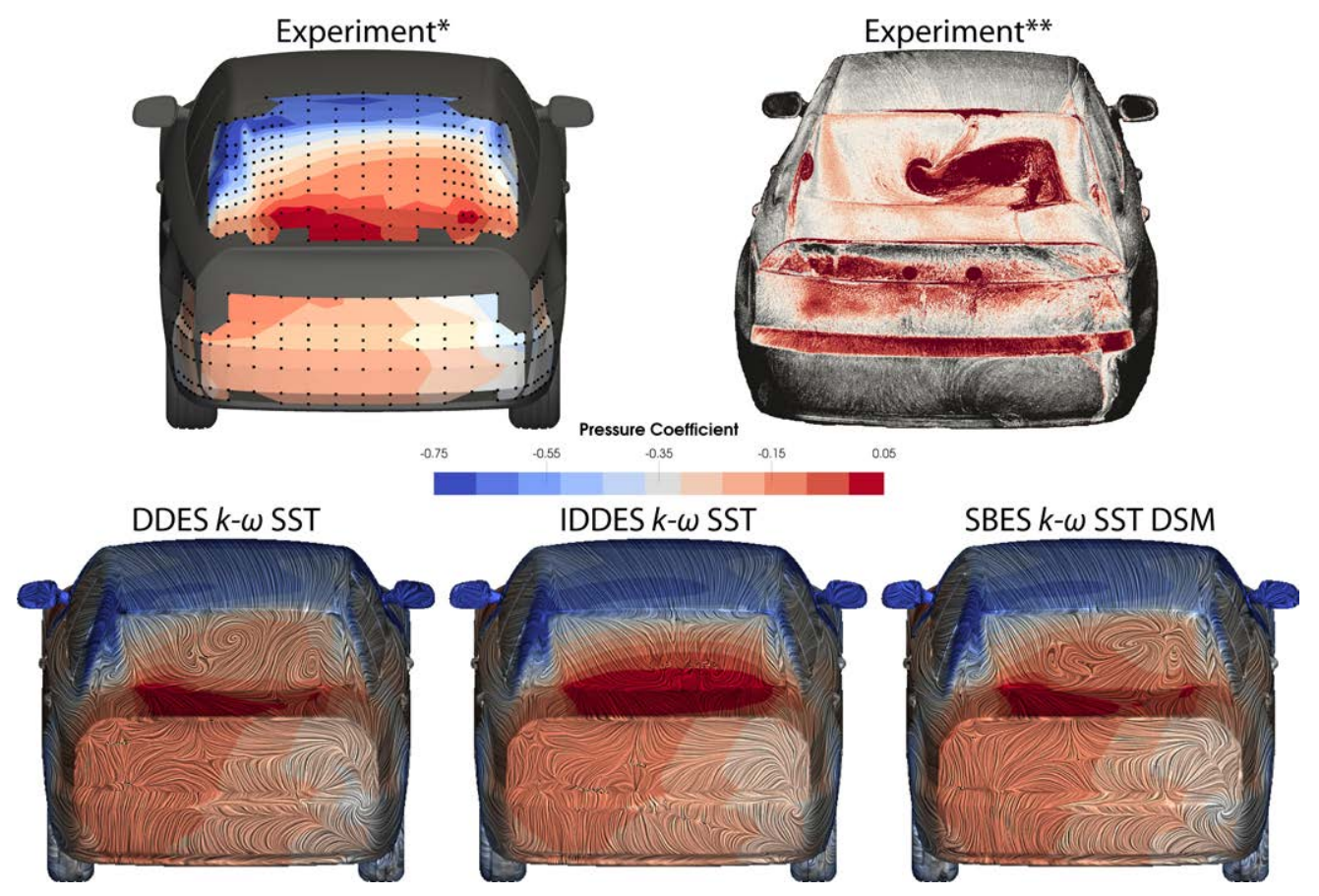

Figure 51: The near-wall flow structures and pressure distribution of the rear of the DrivAer notchback body at $5^{\circ}$ yaw for the measurements, and the three investigated hybrid RANS-LES models. The near-wall flow is achieved from oil film visualization $(* *)$ in the measurement and the wall shear stress field for the simulations. Black points represent pressure probe locations in the measurement $(*)$. Good agreement to the measurement for both near-wall flow patterns and pressure distribution is seen for the SBES $k-\omega$ SST DSM model.

Both the DDES and IDDES models achieve symmetric pressure distribution and near-wall flow for $0^{\circ}$ yaw, although very different, Figure 50. The DDES model overpredicts the separation at the rear window, resulting in two counter-rotating vortices for the nearwall region and lower pressure over the rear window, which is the reason for the higher drag and lift forces. The opposite behavior is seen for the IDDES model, where only a slight separation is seen for the middle part of the rear window, resulting in higher pressure and lower lift force. Asymmetric pressure distribution and near-wall flow behavior is seen for the SBES model. The near-wall flow behavior is in good agreement with the oil film visualization, as the large asymmetric flow structure, with all its rotating focis, in the central part of the rear window is captured. This results in slightly higher pressure than for the DDES model, over the central section of the rear window, agreeing with the measurement. For the base, all the models achieve similar results and are in good agreement with the pressure measurement. This indicates low sensitivity of the rear 


\section{Important Factors for Accurate Scale-Resolving Simulations of Automotive Aerodynamics}

window flow field for the near wake behavior of the DrivAer notchback car body, which is also seen when comparing the near wake behavior between the notchback and the fastback DrivAer configurations. Similar behavior is seen for the models at $5^{\circ}$ yaw, Figure 51, and throughout the yaw sweep. The DDES and SBES models achieve similar pressure and near-wall flow behavior for the leeward side (right) of the rear window, as also seen in the measurement, which is strongly affected by the separation over the central section of the rear window and the leeward side A-pillar vortex.

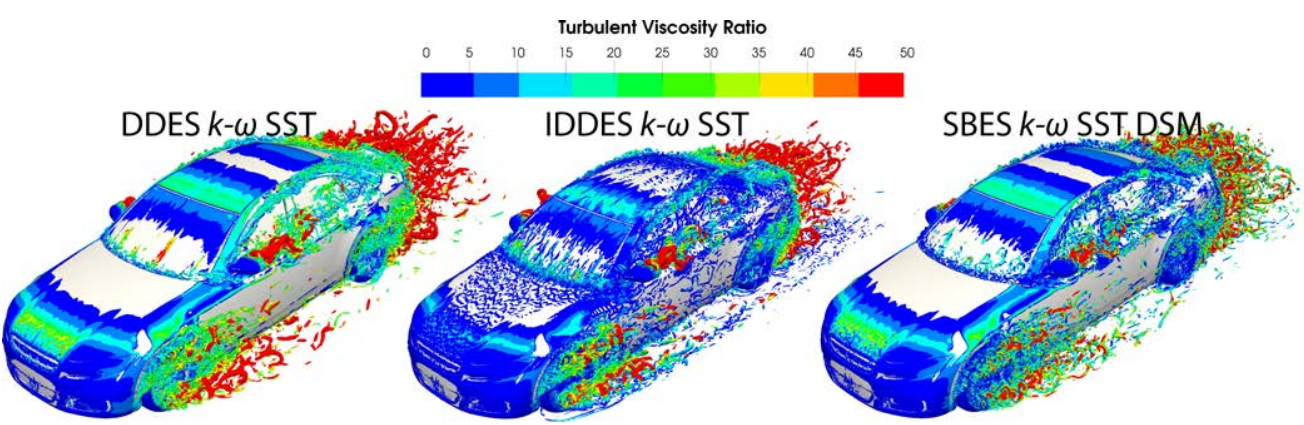

Figure 52: Coherent turbulent structures $\left(Q=3 \cdot 10^{6} \mathrm{~s}^{-2}\right)$ colored with the turbulent viscosity ratio for the three investigated hybrid RANS-LES models for the DrivAer notchback body at $0^{\circ}$ yaw. More turbulent structures are seen for the IDDES $k-\omega$ SST in the near-wall region at the bonnet, as it prematurely switches to WMELS mode. Significantly lower turbulent viscosity is seen in the wakes for the SBES $k-\omega$ SST DSM model, as it more rapidly can switch between the RANS and LES regions.

The reason for the SBES models well agreement to the wind tunnel measurement is believed to be an effect of the more rapid transition between the RANS and LES regions and the strong shielding of the RANS region, resulting in more resolved turbulent structures, as the model otherwise is similar to the IDDES model. The faster transition to the LES region for the SBES model results in lower turbulent viscosity, making it possible to resolve more turbulent structures in the LES region, which otherwise would be suppressed by the turbulent viscosity, Figure 52. More turbulent structures (visualized with the Q-criterion) is seen for the IDDES model when compared to the DDES model. This is due to the IDDES model's ability to switch to WMLES mode when enough upstream turbulence exists. However, the IDDES model, especially with the $k-\omega$ SST model, is seen to be very sensitive and switches early to the WMLES mode. This can, and does, for this case, lead to underresolved turbulence in the near-wall region if the mesh is not fine enough, resulting in incorrect near-wall and turbulence behavior (seen as too low skin friction in Figure 13 in Paper VI). Unfortunately, it does not only lead to incorrect Reynolds stresses but also lead to an incorrect prediction of the sizes for the turbulent structures, which can lead to both under- and over predictions of separations [103]. To achieve sufficient resolving of the near-wall turbulence, the near-wall mesh needs to fulfill, or at least be significantly closer to, the recommended LES near-wall resolution for $s^{+}$and $l^{+}$[63], meaning, for this case, a need of 15 times smaller edge length of each surface elements and as a result a much higher computational cost. These mesh resolution requirements are usually fulfilled, or at least 
close to, with hybrid RANS-LES meshes in regions downstream of separations consisting of recirculating flow with low velocities. For these regions, the meshes are typically refined for better capturing of the resolved turbulence, which also ensures more accurate WMLES behavior. This is also the rationale with the IDDES model, to maintain the unsteadiness where otherwise a RANS model may suppress it, and thereby increasing the accuracy of the model [61].

It should be noted that the mesh sensitivity study is performed with the SBES $k-\omega$ SST DSM model, which might favor the results for the SBES model compared to the DDES and IDDES models. However, as the SBES model almost always can guarantee sufficient shielding of the RANS region, there is a much lower risk that the GIS might affect the results. Hence, the convergence of the results of the mesh sensitivity, without changes in the turbulence models behavior, is achieved for both the RANS and LES regions, which most likely would not be possible to obtain with the DDES and IDDES models.

\subsection{Calibration of IDDES}

To delay the premature switch to WMLES for the IDDES $k-\omega$ SST model the $C_{d 1}$ constant is increased from its default value of 20 [104, 105] in step of 5 up to 40. In Figure 53 , the instantaneous skin friction magnitude for the notchback DrivAer body at $5^{\circ}$ yaw is seen for the IDDES $k-\omega$ SST model with default settings $\left(C_{d 1}=20\right)$ and increased with $C_{d 1}=40$, and compared to the SBES $k-\omega$ SST DSM model.

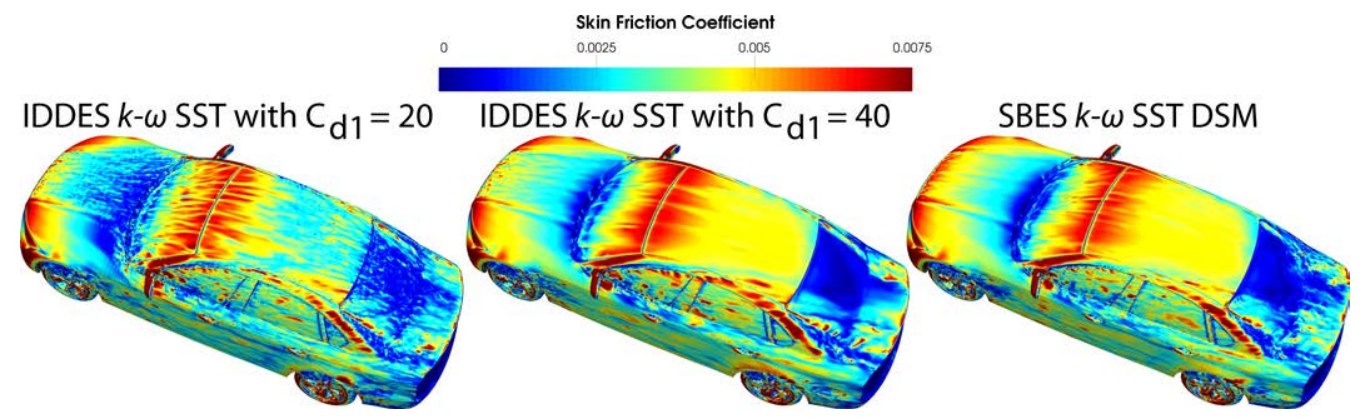

Figure 53: The instantaneous skin friction for the IDDES $k-\omega$ SST model with two different values for the $C_{d 1}$ constant, compared to the SBES $k-\omega$ SST DSM model for the DrivAer notchback body at $5^{\circ}$ yaw. A higher value of the $C_{d 1}$ constant delay the premature switch to WMLES but, unfortunately, result in less unsteadiness for the rear part of the car body where the WMLES mode can be useful. The SBES model can enforce the RANS region and switch to WMLES for the rear part of the car body.

High unsteadiness and lower skin friction is seen for the IDDES $k-\omega$ SST model with $C_{d 1}=20$, especially for the bonnet and roof, compared to the other two. Increasing $C_{d 1}$ reduces the unsteadiness, as it delays the WMLES switch, and increase the skin friction for these regions, but also reduce the unsteadiness in the near-wall region at the rear of 


\section{Important Factors for Accurate Scale-Resolving Simulations of Automotive Aerodynamics}

the car body, where it essentially is wanted. This makes the IDDES model behavior move towards the DDES model, which does not have a WMLES mode, thereby reducing the possible benefit of it. The SBES model, however, achieves both strong RANS shielding and unsteadiness over the rear part of the car body, although not as pronounced as seen for the IDDES model with default settings $\left(C_{d 1}=20\right)$.

\subsection{Sensitivity of Stress Blending and SGS Modeling}

SGS modeling can, as previously seen, have a significant effect on the near-wall flow for wall resolved LES. As mainly RANS modeling is applied for the near-wall region for hybrid RANS-LES models, less effect of the SGS modeling is expected. However, some differences can be seen for the Ahmed body at low Reynolds number, Figure 28 panel b, where significant effects of the laminar-turbulent transitioning may be the main reason for its sensitivity. Therefore, less effect is also to be expected for the higher Reynolds numbers used within the automotive industry.

The SGS modeling investigated for the notchback DrivAer car body at $5^{\circ}$ yaw with the SBES $k-\omega$ SST model, Figure 54. Only small differences are seen for the flow field and the accumulated drag, as less than 0.2 and 4.1 drag counts are seen for the SM and WALE SGS models, respectively, compared to the DSM SGS model. Almost all of the drag force difference for the WALE SGS model is from a slight pressure difference at the leeward side of the base surface. This is due to a rear wake vortex is slightly closer to the base when using the WALE SGS model, migrating its lower pressure onto the base surface. This is believed to be due to the DSM SGS model is slightly less diffusive, as an effect of the dynamic variable $C_{d s}$, resulting in slightly more resolved turbulent kinetic energy upstream the wake on the leeward side of the car body. This causes a slightly narrower wake and force the vortex further away from the base than seen for the WALE SGS model, Figure 54 panel b.

The stress blending is seen to have a marginal effect on the results, as less than 2 drag counts difference occurs between the SDES and SBES models, Figure 54 panel a. The difference mainly originates from the wheels where small turbulence structures exist. This indicates that the strong RANS shielding for the SDES and SBES models is the essential feature for these models. The shielding function, $f_{S D E S}$, is the main secret behind these models, making it possible to more quickly transit between the RANS and LES regions without losing the RANS shielding. Another important feature, especially for industrial use, is that the model is much more robust and reliable in its behavior than seen for the DDES and IDDES models. The SBES model will almost always ensure a protected RANS region and does not prematurely switch to WMLES, even when using with LES fine meshes. This makes it easier for the user to understand what the turbulence model will deliver. The DDES and IDDES models are good and can provide high accuracy. However, these models require more of the user to ensure that they are operating in the state they are designed for and that the user expects and wants, as the risk for suppressed RANS region and premature WMLES switching exists. 

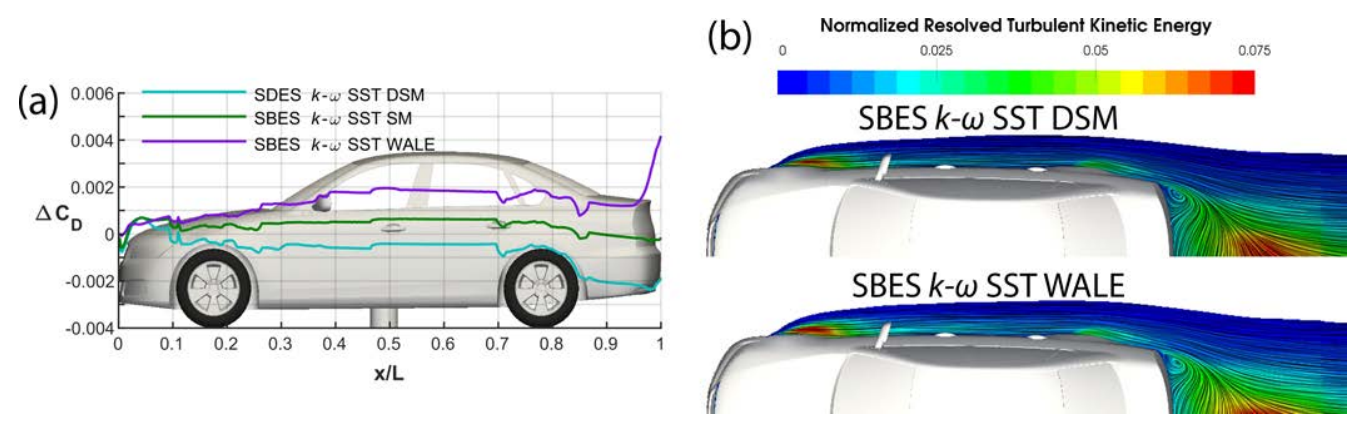

Figure 54: (a) The difference for the accumulated drag against the SBES $k-\omega$ SST DSM model for the SDES $k-\omega$ SST DSM model and the SBES $k-\omega$ SST with SM and WALE SGS models. The simulations are performed for the DrivAer notchback body at $5^{\circ}$ yaw. Only small differences for drag are seen for different SGS modeling, except at the base when using the WALE SGS model. (b) This is due to slightly less resolved turbulent kinetic energy is seen upstream the base on the leeward side of the body, causing a vortex to move closer to the base and lower the base pressure.

\subsection{Accurate Predictions of Small Geometrical Changes}

Even though the accuracy for predicting, especially, drag is good for the DDES, IDDES and SBES models, significant differences during aerodynamic development can still occur. To highlight this, a shark fin antenna is added just upstream the rear window of the DrivAer notchback car body and is investigated for $5^{\circ}$ yaw, Figure 55 . The shark fin will affect the flow over the rear window and is investigated with the DDES $k-\omega$ SST and SBES $k-\omega$ SST DSM models. The results are compared against the original geometrical configuration for each turbulence model.
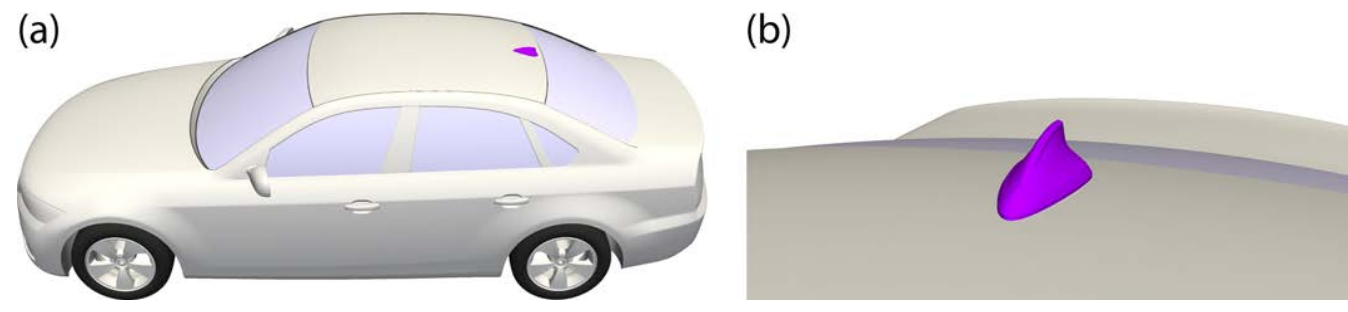

Figure 55: (a) Addition of a shark fin antenna, colored in purple, on the DrivAer notchback body just upstream the rear window. (b) Detailed view on the shark fin antenna.

The models predict opposite drag changes, as the DDES model predicts 3.4 drag counts reduction, while the SBES model sees a 2 drag counts increase when adding the shark fin antenna, Figure 56 panel a. The small shark fin acts as a vortex generator upstream, causing less separation for the DDES model, being the reason for the seen drag reduction for the model, Figure 57. Similar behavior, as for the drag change, is seen for the change of the lift force, Figure 56 panel b. 

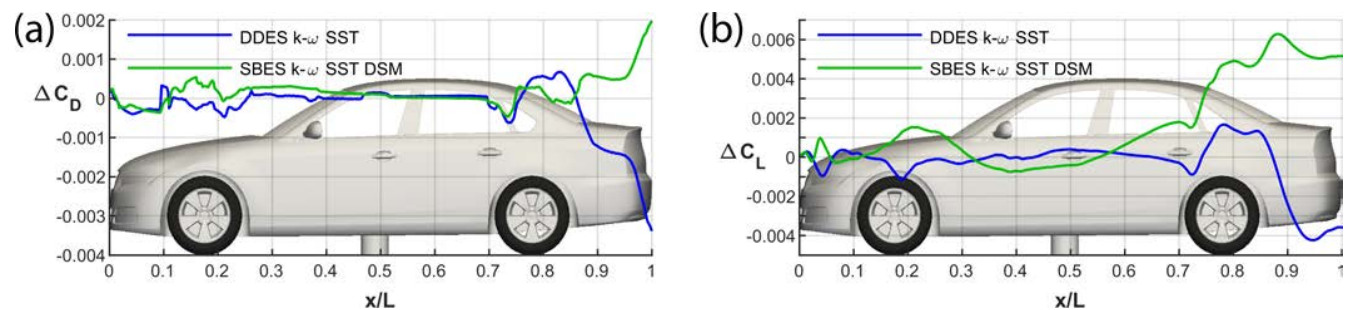

Figure 56: Differences of the accumulated drag (a) and lift (b) forces when adding the shark fin antenna upstream the rear window for the DrivAer notchback body at $5^{\circ}$ yaw. Different prediction of the changes of both drag and lift is seen for the DDES $k-\omega$ SST and SBES $k-\omega$ SST DSM models.

Even though the actual change of the drag force is small, the prediction of the trend is of high importance. This kind of small geometrical changes may be common during aerodynamic development, especially in the later stages, and different trend predictions can lead engineers into making wrong decisions and to late and costly design changes.

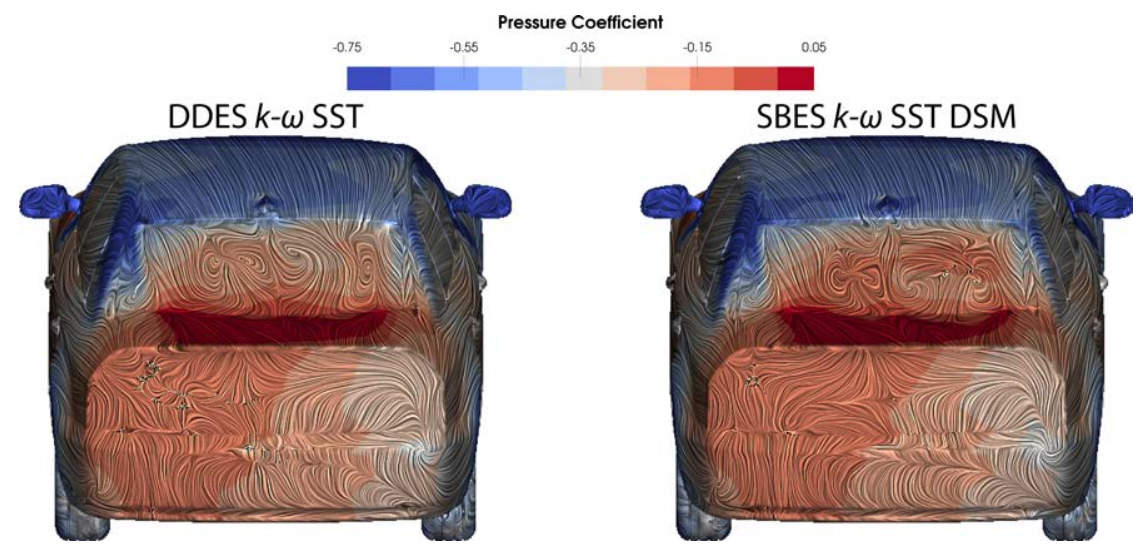

Figure 57: Near wall flow structures and pressure distribution of the rear of the DrivAer notchback body at $5^{\circ}$ yaw fitted with the shark fin antenna for the DDES $k-\omega$ SST and SBES $k-\omega$ SST DSM models. Less separation occurs for the DDES $k-\omega$ SST model with the addition of the shark fin antenna, resulting in less drag. 


\subsection{Intuitive Visualization of Turbulence}

Compared to RANS, SRS enables more valuable flow field information with accurately described flow physics. E.g., from the Reynolds stresses, it is possible to calculate the states of the turbulence for better understanding of the turbulent flow field behavior, thereby better understanding of the flow physics around vehicles. This also enables a better understanding of the performance for the turbulence modeling, and where weaknesses might exist. One of the main reasons behind the lower accuracy for RANS models is connected to the Boussinesq assumption of isotropic behavior of the Reynolds stresses, which might often not be an accurate assumption for aerodynamic flows.
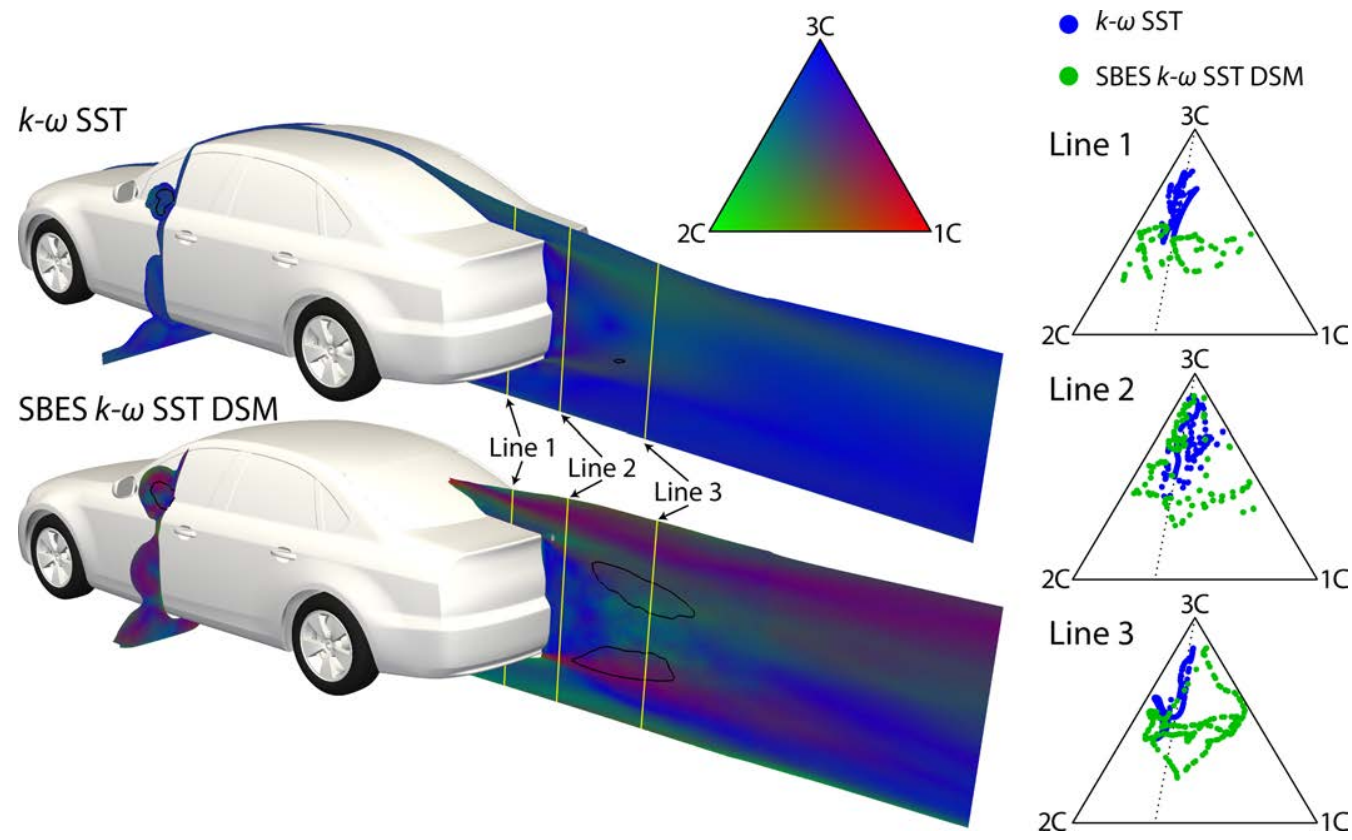

Figure 58: The time-averaged turbulence anisotropy colored with a barycentric colormap for the DrivAer notchback body at $0^{\circ}$ yaw, simulated with both the $k-\omega$ SST (URANS) and SBES $k-\omega$ SST DSM models. Only the LES region is included for the SBES $k-\omega$ SST DSM model. Much more anisotropic behavior of the turbulence is seen for the SBES $k-\omega$ SST DSM model in the $y=0$ and $y z$ planes and the three lines in the wake. For the $k-\omega \mathrm{SST}$ model, mainly isotropic turbulence exist. Black lines show the region with high turbulent kinetic energy $\left(k / U_{\infty}^{2}=0.05\right)$, mainly occurring in the anisotropic turbulence for the SBES $k-\omega$ SST DSM model. The $k-\omega$ SST greatly underpredicts the turbulent kinetic energy in the wakes.

In Figure 58, the turbulent componentality, colored with a barycentric colormap, is seen for the $y=0$ plane and a $y z$ plane just downstream the mirrors for the $k-\omega$ SST and SBES $k-\omega$ SST DSM models. The $k-\omega$ SST is simulated as URANS with the $\Delta \cdot 100$ time-step size. This visualization technique makes it possible to see the behavior of the turbulent 


\section{Important Factors for Accurate Scale-Resolving Simulations of Automotive Aerodynamics}

flow field directly with its location. Few studies have investigated the anisotropic behavior of turbulence for flows around vehicles. Where it has been done [106], it has been done with visualization within the Lumley triangle, making it difficult to interpret the results. Isotropic (3C) behavior is seen for the $k-\omega$ SST model, where only low anisotropy is seen for the shear layers of the rear wake. Even though somewhat green regions exist, it can be seen for the three lines in the wake that the $k-\omega$ SST model mainly acts in the isotropic corner of the barycentric map and also only follows the plain-strain line (dotted line). Similar behavior is also seen in [80] for contracting-diverging nozzle flow simulated with RANS.

For the SBES $k-\omega$ SST DSM model, only the LES region is post-processed, thereby removing the near-wall behavior of the $k-\omega$ SST model from the results. Significantly more turbulence anisotropy is seen, compared to URANS simulation with the $k-\omega$ SST model, especially for the shear layers that are in an intermediate state of $3 \mathrm{C}$ and $1 \mathrm{C}$ turbulence. Only the near wake is seen to be fully isotropic, surrounded by the anisotropic shear layers. Similar behavior is also seen in [106], where the Ahmed body was simulated with LES. In the $y z$ plane just downstream the mirrors, a broad mix of the turbulent states are seen. Interestingly, the higher turbulent kinetic energy (black lines in Figure 58 ) occurs mainly in the anisotropic part of the turbulence, where the shear layers are spreading. Here, the $k-\omega$ SST model significantly underpredicts the turbulent kinetic energy compared to the SBES $k-\omega$ SST DSM model, as only a small region of high turbulent kinetic energy exists in the wake.

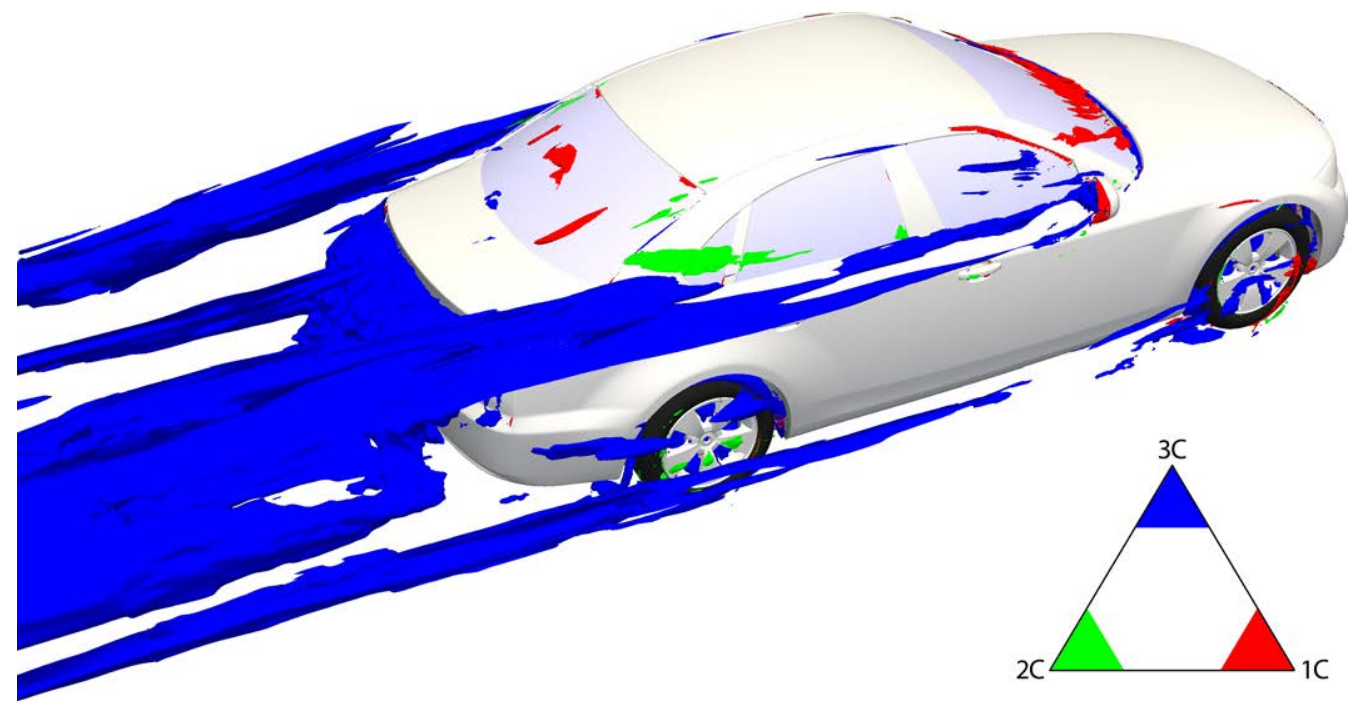

Figure 59: Isosurfaces of the top $30 \%$ of each turbulence componentality for the DrivAer notchback car body at $0^{\circ}$ yaw, simulated with the SBES $k-\omega$ SST DSM model. Isotropic turbulence (3C) is the dominating turbulence componentality, especially in the wakes. However, significant regions of $1 \mathrm{C}$ and $2 \mathrm{C}$ turbulence also exist. 
By creating isosurfaces of the regions with the top $30 \%$ of each turbulent componentality, the dominating turbulent componentalities can be seen. In Figure 59, this is done for the SBES $k-\omega$ SST DSM model, and it is seen that the isotropic turbulence is the dominating turbulent component in terms of volume of the turbulent kinetic energy. Significant regions of $1 \mathrm{C}$ turbulence also exist, especially for the windshield and A-pillar vortices. Some general behavior of the turbulence field around bluff bodies can be seen for the turbulence behavior around the mirror, Figure 59, where the near wake is dominated by isotropic turbulence (3C) and is covered by $1 \mathrm{C}$ shear layers; further downstream, the wake consistently becomes more isotropic (3C).

The behavior of turbulence may have a significant impact on the transport of particles, and might, therefore, be of use for aerodynamic soiling applications. Turbulence can also cause the flow to stay more attached but is also responsible for pressure losses. A specific componentality of the turbulence might, therefore, be more suitable for achieving attached flow with reduced pressure losses. With this visualization tool, a better understanding of the flow physics around vehicles is possible, enabling more possibilities for potential development and tuning of turbulence models suited for automotive aerodynamics. 



\section{Concluding Remarks}

\subsection{Conclusions}

Turbulence modeling is an essential factor in achieving accurate predictions of the external flow around vehicles. Even though only a small part of the turbulence is modeled in LES, the modeling can cause significant differences in the results. This is especially true for the near-wall region, being the holy grail of turbulence modeling, where the SGS modeling is crucial to achieve correct laminar and/or turbulent behavior. The WALE and DSM SGS models are indeed able to predict laminar to turbulent transition, which is shown to be of significant importance for predicting the flow field around the bluff bodies at low Reynolds numbers. LES with either of these SGS models can achieve very high accuracy, as excellent agreement to high-resolution PIV measurements is seen. However, the computational resources needed for running LES at industrially relevant Reynolds numbers for automotive aerodynamics are, unfortunately, at present and even in the near future unfeasible. For the other side of the turbulence modeling spectrum, where all turbulence is modeled, the computational cost is rarely an issue. Instead, the RANS models are based on fundamental assumptions of the turbulence physics, which severely limits their accuracy. Reasonable accuracy is, however, achievable under certain conditions and for specific geometries, making it difficult to achieve consistent and thereby reliable accuracy. This does not mean that RANS models are useless during aerodynamic development but need to be carefully used for conditions where they are reasonably accurate and trustworthy. This is demonstrated in Paper III, where significant geometrical changes to the swap body resulted in a substantial reduction of drag and fuel consumption. For analysis of both geometrical and flow details, especially in regions with and/or affected by high unsteadiness, SRS is needed for reliable predictions.

The small turbulent structures within the near-wall region is the main reason for the high computational cost with LES. By modeling this region with RANS, a much coarser mesh can be used, while still keeping reasonably high accuracy and reducing the computational cost. The temporal resolution does, unfortunately, still keep the computational cost rather high compared to RANS. However, using larger time-step sizes than recommended in 


\section{Important Factors for Accurate Scale-Resolving Simulations of Automotive Aerodynamics}

general best-practice guides, reduces the computational cost greatly. Accuracy is not especially sensitive to the time-step size, as even very large time-steps results in satisfying accuracy for predictions of the drag force. Significant changes locally can, however, be seen when being aggressive with the time-step size, which more often show effects on the prediction of the lift force. Larger steps in time is, therefore, an efficient approach to reduce the computational cost and simulation turnaround time, without significant losses of the accuracy. Reducing the mesh size can also reduce the computational cost greatly, but with more significant deficits to both accuracy and geometrical representation.

Many hybrid RANS-LES models and variations of different complexity exist. The RANS model used for the near-wall region is of high importance. RANS models that generate high turbulent viscosity in the near-wall region, e.g., the realizable $k-\varepsilon$ model, are not suitable, as too much suppression of the resolved turbulence occurs. The shielding of the RANS region is also of high importance, to prevent the LES model from being active within the near-wall region and causing under-resolved turbulence, possibly leading to GIS. This is an issue for models based on the DES methodology, and even shielding improvements as the DDES models struggle with conserving the RANS regions for appropriate hybrid RANSLES meshes. The shielding function in SDES and SBES is, however, able to conserve the RANS region, even on LES-like meshes, making it much stronger than seen for the DDES model. The transitioning between the RANS and LES regions is also of high importance, to minimize the gray-area where the model is neither in full RANS (modeling) nor LES (resolving) mode. The SDES model and, in particular, the SBES model can achieve this faster than the DDES model, as the strong RANS shielding enables a more aggressive definition of the grid length scale, being exceptionally efficient for shear layers. The IDDES model can also achieve a rapid transition to the LES region, but only when being in the WMLES mode as resolved scales then already exist within the near-wall region. Premature switching to WMLES mode is, however, seen for the IDDES model, resulting in underresolved turbulence and too low skin friction. The SDES and SBES models also include a WMLES mode, but no premature switching to it is observed. Which SGS model to use in the LES region is of less importance, as only small differences are seen.

The SBES model achieves high accuracy, and, possibly more important, reliable accuracy for several different vehicle geometries and flow conditions, ranging from simplified generic models (Ahmed body and Allan body) to the industrial realistic DrivAer car body. The strong RANS shielding and the rapid switch to the LES region for the SBES model make it robust and easy to understand for users, as it most likely will behave as expected. The SAS, DDES and IDDES models cannot guarantee this robustness, as especially the mesh resolution may cause different behavior (with too early switching to LES or WMLES) of the models. The DDES and IDDES models can deliver very accurate results, sometimes even more accurate than the SBES model, but result in the uncertainty of not knowing if the model is behaving as expected. This makes the SBES model well suitable for use in an (in particular automated) industrial aerodynamic development process.

Accurate SRS methods also enable a lot more valuable information compared to RANS models, the behavior and componentality of the turbulence, variables not usually used in aerodynamic development. A better understanding of the turbulent flow field may enable for more efficient design, not just for reducing aerodynamic drag but also for other 
applications like soiling and aeroacoustic noise.

With the continuing introduction of electric vehicles, efficient aerodynamics is even more important to ensure vehicles with a satisfying range. With more and more efficient aerodynamic design and introduction of regulations such as WLTP, there is a high need for accurate and fast aerodynamic simulations, which can capture small and large changes of the geometry and flow conditions.

\subsection{Outlook}

The future will continuously need more accurate and faster simulations, and the automotive industry is a driving force for this in their strive to design more efficient and better vehicles. Further and stricter regulations of emissions, like WLTP, will also put higher demands on the accuracy of simulations. For meeting these demands, virtual development will need to keep increasing, and higher prediction certainties from simulations is needed to maintain the increase.

With increasing efficiency and complexity in vehicle design, continuously more design targets need to be addressed simultaneously during the development phase. This can include factors such as cooling, soiling, aeroacoustic noise, aeroelasticity, moving geometries, and upstream turbulence that needs to be considered during the aerodynamic design phase. Many of these factors include transient phenomena, urging the need for continuingly less computationally costly and still accurate SRS methods. In particular, as many of these factors put even higher demands on the predictions of the turbulence behavior as it affects the transport of heat and particles.

Uncertainty quantification (UQ) may be a step towards achieving fewer uncertainties, both in aerodynamic design and even in simulation methodology development. However, UQ can require a tremendous amount of data points, thereby simulations. Steady-state RANS simulations may, for a long time coming to be the only option for achieving this and raise questions of how to improve the reliable accuracy of the turbulence modeling. Using the turbulent behavior from accurate SRS and experiments to move away from isotropic Reynolds stresses by the use of Reynolds stress perturbation may be one option, while the use of artificial intelligence for improving or define new RANS models is another interesting approach.

There exist several other approaches to solve these problems, where many show promising trends in terms of low computational cost and high accuracy. For example, the lattice Boltzmann method show improving trends, especially on GPU's, but also needs to rely on the modeling of the turbulence for the near-wall region, still being (and likely will be) the holy grail of CFD. 



\title{
Review of Appended Papers
}

\section{Paper I}

\begin{abstract}
Aerodynamic Drag Reduction - from Conceptual Design on a Simplified Generic Model to Full-Scale Road Tests, Petter Ekman, Roland Gårdhagen, Torbjörn Virdung, Matts Karlsson, SAE Technical Paper 2015-01-1543, 2015.

This paper investigates several different RANS turbulence models with different wall functions for the prediction of the drag force of a simplified truck model in different geometrical configurations. The results are compared to wind tunnel measurements and show a wide mix of accuracy between the turbulence models and wall functions. The realizable $k-\varepsilon$ model with the EWT shows good correlation to the wind tunnel measurements for a specific range of gap distances and is used for a design of experiment study of adding a radius on the top leading edge of the swap body for different gap distances. The modification is added to a full-scale truck and showed a $3 \%$ fuel reduction during a test-period.
\end{abstract}

Author Contributions: Ekman did all the simulation work, post-processing, analysis and writing. Gårdhagen, Virdung and Karlsson have contributed by providing comments and discussion through the work.

\section{Paper II}

Importance of Sub-Grid Scale Modelling for Accurate Aerodynamic Simulations, Petter Ekman, James Venning, Torbjörn Virdung, Matts Karlsson, Accepted for Publication in ASME Journal of Fluids Engineering, March 2020.

In this paper, the flow around the Ahmed body is investigated with LES and compared to high-resolution PIV measurements. Particular emphasis is on the ability of three commonly used SGS models to capture the near-wall behavior, small-scale dissipation, and the behavior of the rear wake. It is seen that this is crucial for achieving good correlation to the PIV measurements, thereby showing the importance of the SGS modeling. The 


\section{Important Factors for Accurate Scale-Resolving Simulations of Automotive Aerodynamics}

best agreement is for the dynamic Smagorinsky-Lilly SGS model, while the Wall-Adopting Local Eddy-Viscosity SGS model is not far off, as both SGS models can capture correct near-wall flow behavior.

Author Contributions: Ekman did all the simulation work, post-processing of simulations and experiment (from Venning), analysis and writing. Venning, Virdung and Karlsson have contributed by providing comments and discussion through the work.

\section{Paper III}

Aerodynamic Drag Reduction of a Light Truck - from Conceptual Design to Full Scale Road Tests, Petter Ekman, Roland Gårdhagen, Torbjörn Virdung, Matts Karlsson, SAE Technical Paper 2016-01-1594, 2016.

There exist significant possibilities to reduce the aerodynamic drag and hence the fuel consumption for light trucks consisting of parts from several manufacturers. Rarely is the aerodynamics of the final shape considered for these vehicles. In this paper, the swap body of a light truck is modified in order to reduce the aerodynamic drag but still contain sufficient loading volume, by use of CFD. With small modifications, the drag is reduced with $28.4 \%$, with only small losses to the loading volume. A prototype swap body was manufactured and resulted in a $12 \%$ reduced fuel consumption during a testing period.

Author Contributions: Ekman did all the simulation work, post-processing, analysis and writing. Gårdhagen, Virdung and Karlsson have contributed by providing comments and discussion through the work.

\section{Paper IV}

\section{Aerodynamics of an Unloaded Timber Truck - A CFD Investigation \\ Petter Ekman, Roland Gårdhagen, Torbjörn Virdung, Matts Karlsson \\ SAE International Journal of Commercial Vehicles. 9(2):2016, 2016.}

One special case of goods transport is the transport of timber with the use of timber trucks. Timber trucks have $30 \%$ higher fuel consumption than ordinary tractor-trailer configurations. One possible reason for this is the lack of aerodynamic development of these trucks. This paper investigates the flow around an unloaded timber truck and how much the aerodynamic drag can be reduced with the addition of a shield between the bulkhead and first stake pair. The drag reduction shield resulted in weaker vortical structures in the wake, thereby increased base pressure and leading to a $6.7 \%$ reduction of drag.

Author Contributions: Ekman did all the simulation work, post-processing, analysis and writing. Gårdhagen, Virdung and Karlsson have contributed by providing comments and discussion through the work. 


\title{
Paper V
}

\author{
Accuracy and Speed for Scale-Resolving Simulations of the DrivAer \\ Reference Model, Petter Ekman, Torbjörn Larsson, Torbjörn Virdung, Matts Karlsson, \\ SAE Technical Paper 2019-01-0639, 2019.
}

In the move to increase the accuracy of aerodynamic simulations within the automotive industry, many automotive manufacturers switch from steady-state RANS simulations to scale-resolving simulations, resulting in considerably increased computational cost. A significant contributor to the increased cost is the need for small time-step sizes. In this paper, the effect of using a much larger time-step size than recommended in best practice guides is investigated for computational cost and accuracy. A significant reduction of computational cost $(>90 \%)$ is possible with kept accuracy of aerodynamic forces and flow field.

Author Contributions: Ekman did all the simulation work, post-processing, analysis and writing. Virdung wrote a section of the introduction and have, together with Larsson and Karlsson contributed by providing comments and discussion through the work.

\section{Paper VI}

\begin{abstract}
Assessment of Hybrid RANS-LES Methods for Accurate Automotive
Aerodynamic Simulations, Petter Ekman, Dirk Wieser, Torbjörn Virdung, Matts Karlsson, Submitted for Publication, December 2019.

In this paper, three commonly used hybrid RANS-LES models are compared to wind tunnel measurements of the DrivAer notchback and fastback car bodies. A yaw angle sweep up to $7^{\circ}$ yaw is conducted, to see how accurate the models are for changing conditions. Particular focus is on the complex flow over the rear window, being the region where the models differ the most. Only the SBES $k-\omega$ SST DSM can capture the asymmetric flow field over the rear window and is in good agreement with the wind tunnel measurements. The strong shielding for the SBES model, also makes it superior in terms of robustness, as the DDES and IDDES models see failed RANS shielding and premature switching to WMLES mode, respectively.
\end{abstract}

Author Contributions: Ekman did all the simulation work, post-processing of simulations and experiment (from Wieser), analysis and writing. Wieser wrote the Experimental Measurements Section. Virdung and Karlsson have contributed by providing comments and discussion through the work. 



\section{Bibliography}

[1] European Environment Agency. Total greenhouse gas emission trends and projections in Europe. https://www.eea.europa.eu/data-and-maps/indicators/ greenhouse-gas-emission-trends-6/assessment-3, 2019. Last accessed January 2020.

[2] European Commission. Transport emissions. https://ec.europa.eu/clima/ policies/transport_en, 2016. Last accessed October 2019.

[3] Council of the European Union. Cutting emissions: Council adopts $\mathrm{CO}_{2}$ standards for trucks. https://www.consilium.europa.eu/en/press/press-releases/ 2019/06/13/cutting-emissions-council-adopts-co2-standards-for-trucks/, 2019. Last accessed January 2020.

[4] Audi AG. How aerodynamics influence an electric car's range. https: //www .audi.com/en/experience-audi/mobility-and-trends/e-mobility/ e-tron-aerodynamic.html, 2019. Last accessed October 2019.

[5] H. Kawamata, S. Kuroda, S. Tanaka, and M. Oshima. Improvement of Practical Electric Consumption by Drag Reducing under Cross Wind. Technical report, SAE Technical Paper, 2016.

[6] WLTP Facts. What Is WLTP and How Does It Work? https://wltpfacts.eu/ what-is-wltp-how-will-it-work/, 2017. Last accessed October 2019.

[7] UNECE. Global Technical Regulation No. 15 (Worldwide harmonized Light vehicles Test Procedure). https://www.unece.org/fileadmin/DAM/trans/main/ wp29/wp29r-1998agr-rules/ECE-TRANS-180a15e.pdf, 2017. Last accessed January 2021.

[8] UNECE. Global Technical Regulation No. 15 (Worldwide harmonized Light vehicles Test Procedure), Annex $7 \S 3.2 .3 .2 .2 .3$. https://www.unece.org/fileadmin/ DAM/trans/doc/2018/wp29other/EU_2017_1151.pdf, 2017. Last accessed January 2021.

[9] S.R. Ahmed, G. Ramm, and G. Faltin. Some Salient Features of the Time-Averaged Ground Vehicle Wake. Technical report, SAE Technical Paper, 1984. 


\section{Important Factors for Accurate Scale-Resolving Simulations of Automotive Aerodynamics}

[10] G.M. Le Good and K.P. Garry. On the Use of Reference Models in Automotive Aerodynamics. Technical report, SAE Technical Paper, 2004.

[11] A.I. Heft, T. Indinger, and N.A. Adams. Introduction of a New Realistic Generic Car Model for Aerodynamic Investigations. Technical report, SAE Technical Paper, 2012.

[12] O. Delgado and N. Lutsey. The US SuperTruck Program. Washington DC, 2014.

[13] B. Kraaijenhagen, C. van der Zweep, A. Lischke, J. Engasser, P. Elofsson, et al. Aerodynamic and Flexible Trucks for next Generation of Long Distance Road Transport (AEROFLEX). International Forum For Road Transport technology (IFRTT), 2018.

[14] AEROFLEX. AEROFLEX - Aerodynamic and Flexible Trucks for Next Generation of Long Distance Road Transport. https://aeroflex-project.eu/, 2020. Last accessed January 2020.

[15] O. Reynolds. XXIX. An Experimental Investigation of the Circumstances which Determine Whether the Motion of Water Shall be Direct or Sinuous, and of the Law of Resistance in Parallel Channels. Philosophical Transactions of the Royal Society of London, (174):935-982, 1883.

[16] W. Hucho and G. Sovran. Aerodynamics of Road Vehicles. Annual review of fluid mechanics, 25(1):485-537, 1993.

[17] R. Wood. Reynolds Number Impact on Commercial Vehicle Aerodynamics and Performance. SAE International Journal of Commercial Vehicles, 8(2015-01-2859):590$667,2015$.

[18] H. Schlichting and K. Gersten. Boundary-Layer Theory. Springer, 2016.

[19] H.K. Versteeg and W. Malalasekera. An Introduction to Computational Fluid Dynamics: The Finite Volume Method. Pearson Education, 2007.

[20] E. Mercker, N. Breuer, H. Berneburg, and H.J. Emmelmann. On the Aerodynamic Interference due to the Rolling Wheels of Passenger Cars. SAE transactions, pages 460-476, 1991.

[21] C. Landström, T. Walker, L. Christoffersen, and L. Löfdahl. Influences of Different Front and Rear Wheel Designs on Aerodynamic Drag of a Sedan Type Passenger Car. Technical report, SAE Technical Paper, 2011.

[22] Z. Machado, C. Van Wingerden, J. Pappas, B. McAlexander, J. LeMaire, O. Pierrakos, and H. Watson. Increasing Fuel Savings of Class-8 Tractor-Trailers by Reducing Aerodynamic Drag. In 2014 Systems and Information Engineering Design Symposium (SIEDS), pages 6-11. IEEE, 2014.

[23] J.W. Allan. Aerodynamic Drag and Pressure Measurements on a Simplified TractorTrailer Model. Journal of Wind Engineering and Industrial Aerodynamics, 9(12):125-136, 1981. 
[24] J.J. Bertin and M.L. Smith. Aerodynamics for Engineers, volume 5. Prentice Hall Upper Saddle River, NJ, 1998.

[25] L.P. Kadanoff. On Two Levels. In From Order To Chaos: Essays: Critical, Chaotic and Otherwise, pages 11-12. World Scientific, 1993.

[26] H. Tennekes, J.L. Lumley, et al. A First Course in Turbulence. MIT press, 1972.

[27] A.N. Kolmogorov. The Local Structure of Turbulence in Incompressible Viscous Fluid for Very Large Reynolds Numbers. Proceedings of the Royal Society of London. Series A: Mathematical and Physical Sciences, 434(1890):9-13, 1991.

[28] J. Mathieu and J. Scott. An Introduction To Turbulent Flow. Cambridge University Press, 2000.

[29] J. Boussinesq. Théorie de l'écoulement tourbillonnant et tumultueux des liquides dans les lits rectilignes a grande section, volume 1. Gauthier-Villars, 1897.

[30] P.R. Spalart and S. Allmaras. A One-Equation Turbulence Model for Aerodynamic Flows. In 30th Aerospace Sciences Meeting and Exhibit, page 439, 1992.

[31] B.E. Launder and D.B. Spalding. Mathematical Models of Turbulence. Academic press, 1972.

[32] B.E. Launder and B.I. Sharma. Application of the Energy-Dissipation Model of Turbulence to the Calculation of Flow Near a Spinning Disc. Letters in Heat and Mass Transfer, 1(2):131-137, 1974.

[33] B.E. Launder and D.B. Spalding. The Numerical Computation of Turbulent Flows. In Numerical Prediction of Flow, Heat Transfer, Turbulence and Combustion, pages 96-116. Elsevier, 1983.

[34] C. Mockett. A Comprehensive Study of Detached Eddy Simulation. Univerlag TU Berlin, 2009.

[35] T-H. Shih, W.W. Liou, A. Shabbir, Z. Yang, and J. Zhu. A New $k-\varepsilon$ Eddy Viscosity Model for High Reynolds Number Turbulent Flows. Computers $\&$ Fluids, 24(3):227-238, 1995.

[36] D.C. Wilcox. Reassessment of the Scale-Determining Equation for Advanced Turbulence Models. AIAA journal, 26(11):1299-1310, 1988.

[37] F.R. Menter. Two-Equation Eddy-Viscosity Turbulence Models for Engineering Applications. AIAA journal, 32(8):1598-1605, 1994.

[38] F.R. Menter. Review of The Shear-Stress Transport Turbulence Model Experience from an Industrial Perspective. International Journal of Computational Fluid Dynamics, 23(4):305-316, 2009.

[39] M.M. Gibson and B.E. Launder. Ground Effects on Pressure Fluctuations in the Atmospheric Boundary Layer. Journal of Fluid Mechanics, 86(3):491-511, 1978. 


\section{Important Factors for Accurate Scale-Resolving Simulations of Automotive Aerodynamics}

[40] ANSYS Inc. ANSYS FLUENT Theory Guide. Canonsburg, PA, Release 18.0, 2017.

[41] M. Wolfshtein. The Velocity and Temperature Distribution In One-Dimensional Flow With Turbulence Augmentation and Pressure Gradient. International Journal of Heat and Mass Transfer, 12(3):301-318, 1969.

[42] H.C. Chen and V.C. Patel. Near-Wall Turbulence Models for Complex Flows Including Separation. AIAA journal, 26(6):641-648, 1988.

[43] T. Jongen. Simulation and Modeling of Turbulent Incompressible Flows. EPF Lausanne, Lausanne, PhD Thesis, 1992.

[44] W. Rodi, G. Constantinescu, and T. Stoesser. Large-Eddy Simulation in Hydraulics. Crc Press, 2013.

[45] F.R. Menter. Best Practice: Scale-Resolving Simulations in ANSYS CFD. ANSYS Germany GmbH, 1, 2012.

[46] F.R. Menter and Y. Egorov. The Scale-Adaptive Simulation Method for Unsteady Turbulent Flow Predictions. Part 1: Theory and Model Description. Flow, Turbulence and Combustion, 85(1):113-138, 2010.

[47] P. Sagaut. Multiscale and Multiresolution Approaches in Turbulence: LES, DES and Hybrid RANS/LES Methods: Applications and Guidelines. World Scientific, 2013.

[48] F.R. Menter and Y. Egorov. A Scale Adaptive Simulation Model using Two-Equation Models. In 43rd AIAA Aerospace Sciences Meeting and Exhibit, page 1095, 2005.

[49] J. Smagorinsky. General Circulation Experiments with the Primitive Equations: I. The Basic Experiment. Monthly Weather Review, 91(3):99-164, 1963.

[50] S. Krajnović and L. Davidson. Large-Eddy Simulation of the Flow Around Simplified Car Model. Technical report, SAE Technical Paper, 2004.

[51] A. Sohankar. Flow over a Bluff Body from Moderate to High Reynolds Numbers using Large Eddy Simulation. Computers \&f Fluids, 35(10):1154-1168, 2006.

[52] A. Sohankar, L. Davidson, and C. Norberg. Large Eddy Simulation of Flow Past a Square Cylinder: Comparison of Different Subgrid Scale Models. J. Fluids Eng., 122(1):39-47, 1999.

[53] J. Östh and S. Krajnović. The Flow Around a Simplified Tractor-Trailer Model Studied by Large Eddy Simulation. Journal of Wind Engineering and Industrial Aerodynamics, 102:36-47, 2012.

[54] M. Germano, U. Piomelli, P. Moin, and W.H. Cabot. A Dynamic Subgrid-Scale Eddy Viscosity Model. Physics of Fluids A: Fluid Dynamics, 3(7):1760-1765, 1991.

[55] S.E. Kim et al. Large Eddy Simulation Using Unstructured Meshes and Dynamic Subgrid-Scale Turbulence Models. AIAA paper, 2548:2004, 2004. 
[56] F. Nicoud and F. Ducros. Subgrid-Scale Stress Modelling Based on the Square of the Velocity Gradient Tensor. Flow, Turbulence and Combustion, 62(3):183-200, 1999.

[57] L. Davidson, D. Cokljat, J. Fröhlich, M.A. Leschziner, C. Mellen, and W. Rodi. LESFOIL: Large Eddy Simulation of Flow Around a High Lift Airfoil: Results of the Project LESFOIL Supported by the European Union 1998-2001, volume 83. Springer Science \& Business Media, 2012.

[58] J. Fröhlich and D. Von Terzi. Hybrid LES/RANS Methods for the Simulation of Turbulent Flows. Progress in Aerospace Sciences, 44(5):349-377, 2008.

[59] P.R. Spalart. Comments on the Feasibility of LES for Wings, and on a Hybrid RANS/LES Approach. In Proceedings of first AFOSR international conference on DNS/LES. Greyden Press, 1997.

[60] P.R. Spalart, S. Deck, M.L. Shur, K.D. Squires, M.K. Strelets, and A. Travin. A New Version of Detached-Eddy Simulation, Resistant to Ambiguous Grid Densities. Theoretical and computational fluid dynamics, 20(3):181, 2006.

[61] M.L. Shur, P.R. Spalart, M.K. Strelets, and A.K. Travin. A hybrid RANS-LES approach with delayed-DES and wall-modelled LES capabilities. International Journal of Heat and Fluid Flow, 29(6):1638-1649, 2008.

[62] F.R. Menter. Stress-Blended Eddy Simulation (SBES) - A New Paradigm in Hybrid RANS-LES Modeling. In Symposium on Hybrid RANS-LES Methods, pages 27-37. Springer, 2016.

[63] ANSYS Inc. ANSYS FLUENT Theory Guide. Canonsburg, PA, Release 2019R3, 2019.

[64] SAE International Surface Vehicle Recommended Practice. Guidelines for Aerodynamic Assessment of Medium and Heavy Commercial Ground Vehicles Using Computational Fluid Dynamics. SAE International, SAE Standard J2966, 2013.

[65] D. Wieser, H.J. Schmidt, S. Müller, C. Strangfeld, C. Nayeri, and C. Paschereit. Experimental Comparison of the Aerodynamic Behavior of Fastback and Notchback DrivAer Models. SAE International Journal of Passenger Cars-Mechanical Systems, 7(2014-01-0613):682-691, 2014.

[66] D. Wieser, H. Lang, C. Nayeri, and C. Paschereit. Manipulation of the Aerodynamic Behavior of the DrivAer Model with Fluidic Oscillators. SAE International Journal of Passenger Cars-Mechanical Systems, 8(2015-01-1540):687-702, 2015.

[67] J. Venning, D.L. Jacono, D. Burton, M. Thompson, and J. Sheridan. The Effect of Aspect Ratio on the Wake of the Ahmed Body. Experiments in Fluids, 56(6):126, 2015.

[68] J. Venning, D.L. Jacono, D. Burton, M.C. Thompson, and J. Sheridan. The Nature of the Vortical Structures in the Near Wake of the Ahmed Body. Proceedings of the Institution of Mechanical Engineers, Part D: Journal of Automobile Engineering, 231(9):1239-1244, 2017. 


\section{Important Factors for Accurate Scale-Resolving Simulations of Automotive Aerodynamics}

[69] P.R. Spalart and C. Streett. Young-Person"s Guide to Detached-Eddy Simulation Grids. 2001.

[70] U. Piomelli and J.R. Chasnov. Large-Eddy Simulations: Theory and Applications, pages 269-336. Springer Netherlands, Dordrecht, 1996.

[71] S.B. Pope. Ten Questions Concerning the Large-Eddy Simulation of Turbulent Flows. New Journal of Physics, 6(1):35, 2004.

[72] L. Davidson. Large Eddy Simulations: How to Evaluate Resolution. International Journal of Heat and Fluid Flow, 30(5):1016-1025, 2009.

[73] L. Davidson. How to Estimate the Resolution of an LES of Recirculating Flow. In Quality and Reliability of Large-Eddy Simulations II, pages 269-286. Springer, 2011.

[74] L. Graftieaux, M. Michard, and N. Grosjean. Combining PIV, POD and Vortex Identification Algorithms for the Study of Unsteady Turbulent Swirling Flows. Measurement Science and technology, 12(9):1422, 2001.

[75] C. Strangfeld, D. Wieser, H.J. Schmidt, R. Woszidlo, C. Nayeri, and C. Paschereit. Experimental Study of Baseline Flow Characteristics for the Realistic Car Model DrivAer. Technical report, SAE Technical Paper, 2013.

[76] D. Wieser, C. Nayeri, and C. Paschereit. Experiments with Vortex Generators applied to a Notchback Car Model. In 53rd AIAA Aerospace Sciences Meeting, page 1236, 2015.

[77] M. Andersson, T. Ebbers, and M. Karlsson. Characterization and Estimation of Turbulence-Related Wall Shear Stress in Patient-Specific pulsatile Blood Flow. Journal of Biomechanics, 85:108-117, 2019.

[78] J.L. Lumley and G.R. Newman. The Return to Isotropy of Homogeneous Turbulence. Journal of Fluid Mechanics, 82(1):161-178, 1977.

[79] P. Berg, C. Roloff, O. Beuing, S. Voss, S-I. Sugiyama, N. Aristokleous, A.S. Anayiotos, N. Ashton, A. Revell, N.W. Bressloff, et al. The Computational Fluid Dynamics Rupture Challenge 2013 - Phase II: Variability of Hemodynamic Simulations in Two Intracranial Aneurysms. Journal of Biomechanical Engineering, $137(12), 2015$.

[80] M. Emory and G. Iaccarino. Visualizing Turbulence Anisotropy in the Spatial Domain with Componentality Contours. Cent. Turbul. Res. Annu. Res. Briefs, pages 123-138, 2014.

[81] M.A. Emory. Estimating Model-Form Uncertainty in Reynolds-Averaged NavierStokes Closures. PhD thesis, Stanford University, 2014.

[82] M. Islam, F. Decker, E. De Villiers, A. Jackson, J. Gines, T. Grahs, A. Gitt-Gehrke, and J.C. i Font. Application of Detached-Eddy Simulation for Automotive Aerodynamics Development. Technical report, SAE Technical Paper, 2009. 
[83] P.R. Spalart. Strategies for Turbulence Modelling and Simulations. International Journal of Heat and Fluid Flow, 21(3):252-263, 2000.

[84] A. Spohn and P. Gilliéron. Flow Separations Generated by a Simplified Geometry of an Automotive Vehicle. In IUTAM Symposium: Unsteady Separated Flows, pages 8-12. Citeseer, 2002.

[85] S. Krajnović and L. Davidson. Flow Around a Simplified Car, Part 1: Large Eddy Simulation. 2005.

[86] S. Schmidt, M. Franke, and F. Thiele. Assessment of SGS Models in LES Applied to a NACA 4412 Airfoil. In 39th Aerospace Sciences Meeting and Exhibit, page 434, 2001.

[87] C. Moussaed, S. Wornom, M-V. Salvetti, B. Koobus, and A. Dervieux. Impact of Dynamic Subgrid-Scale Modeling in Variational Multiscale Large-Eddy Simulation of Bluff-Body Flows. Acta Mechanica, 225(12):3309-3323, 2014.

[88] National Supercomputer Centre. Tetralith. https://www.nsc.liu.se/systems/ tetralith/, 2019. Last accessed March 2020.

[89] M. Izzo and A. Myhr. Lastbilars Klimateffektivitet och Utsläpp-Rapport 2015: 12;(Trucks Environmental Efficiency and Emissions-Report 2015), 2015.

[90] E. Johannes, P. Ekman, M. Huge-Brodin, and M. Karlsson. Sustainable Timber Transport-Economic Aspects of Aerodynamic Reconfiguration. Sustainability, 10(6):1965, 2018.

[91] N. Ashton, P. Unterlechner, and T. Blacha. Assessing the Sensitivity of Hybrid RANS-LES Simulations to Mesh Resolution, Numerical Schemes and Turbulence Modelling within an Industrial CFD Process. Technical report, SAE Technical Paper, 2018.

[92] N. Ashton, V. Skaperdas, O. Sinclair, et al. 001 - University of Oxford Submission - Case 2a \& Case2b. http://autocfd-transfer.eng.ox.ac.uk/Presentations/ 001-Oxford-Presentation.pdf, 2019. Last accessed March 2020.

[93] E. Guilmineau. Numerical Simulations of Flow Around a Realistic Generic Car Model. SAE International Journal of Passenger Cars-Mechanical Systems, 7(201401-0607):646-653, 2014.

[94] N. Ashton and A. Revell. Comparison of RANS and DES Methods for the DrivAer Automotive Body. Technical report, SAE Technical Paper, 2015.

[95] N. Ashton, A. West, S. Lardeau, and A. Revell. Assessment of RANS and DES Methods for Realistic Automotive Models. Computers \& Fluids, 128:1-15, 2016.

[96] T. Hobeika and S. Sebben. CFD Investigation on Wheel Rotation Modelling. Journal of Wind Engineering and Industrial Aerodynamics, 174:241-251, 2018. 


\section{Important Factors for Accurate Scale-Resolving Simulations of Automotive Aerodynamics}

[97] E. Ljungskog, S. Sebben, and A. Broniewicz. Inclusion of the Physical Wind Tunnel in Vehicle CFD Simulations for Improved Prediction Quality. Journal of Wind Engineering and Industrial Aerodynamics, 197:104055, 2020.

[98] J. Munoz-Paniagua, J. García, and B. Lehugeur. Evaluation of RANS, SAS and IDDES Models for the Simulation of the Flow Around a High-Speed Train Subjected to Crosswind. Journal of Wind Engineering and Industrial Aerodynamics, 171:50-66, 2017.

[99] N. Ashton, V. Skaperdas, et al. Cross-Plotting - Case 2a/b. http: //autocfd-transfer.eng.ox.ac.uk/Presentations/Case2-summary-v2.pdf, 2019. Last accessed March 2020.

[100] A.P. Gaylard, J.P. Howell, and K.P. Garry. Observation of Flow Asymmetry Over the Rear of Notchback Vehicles. Technical report, SAE Technical Paper, 2007.

[101] N.J. Lawson, K.P. Garry, and N. Faucompret. An Investigation of the Flow Characteristics in the Bootdeck Region of a Scale Model Notchback Saloon Vehicle. Proceedings of the Institution of Mechanical Engineers, Part D: Journal of Automobile Engineering, 221(6):739-754, 2007.

[102] D.B. Sims-Williams, D. Marwood, and A.J. Sprot. Links Between Notchback Geometry, Aerodynamic Drag, Flow Asymmetry and Unsteady Wake Structure. SAE International Journal of Passenger Cars. Mechanical Systems., 4(1):156-165, 2011.

[103] F.R. Menter. On the Behavior of Under-Resolved Turbulence. Personal communication, Oxford 2019 December 11th.

[104] M.S. Gritskevich, A.V. Garbaruk, J. Schütze, and F.R. Menter. Development of DDES and IDDES Formulations for the $k-\omega$ Shear Stress Transport Model. Flow, Turbulence and Combustion, 88(3):431-449, 2012.

[105] M.S. Gritskevich, A.V. Garbaruk, and F.R. Menter. Fine-tuning of DDES and IDDES Formulations to the $k-\omega$ Shear Stress Transport Model. Progress in Flight Physics, 5:23-42, 2013.

[106] M. Minguez, R. Pasquetti, and E. Serre. High-Order Large-Eddy Simulation of Flow over the "Ahmed Body" Car Model. Physics of Fluids, 20(9):095101, 2008. 



\section{Papers}

The papers associated with this thesis have been removed for copyright reasons. For more details about these see:

http://urn.kb.se/resolve?urn=urn:nbn:se:liu:diva-164926 

